\title{
WestVirginiaUniversity
}

THE RESEARCH REPOSITORY @ WVU

Graduate Theses, Dissertations, and Problem Reports

2016

\section{An integrated lateral flow device for flow control and blood separation}

\author{
Veronica Betancur Calle
}

Follow this and additional works at: https://researchrepository.wvu.edu/etd

\section{Recommended Citation}

Betancur Calle, Veronica, "An integrated lateral flow device for flow control and blood separation" (2016). Graduate Theses, Dissertations, and Problem Reports. 7064.

https://researchrepository.wvu.edu/etd/7064

This Thesis is protected by copyright and/or related rights. It has been brought to you by the The Research Repository @ WVU with permission from the rights-holder(s). You are free to use this Thesis in any way that is permitted by the copyright and related rights legislation that applies to your use. For other uses you must obtain permission from the rights-holder(s) directly, unless additional rights are indicated by a Creative Commons license in the record and/ or on the work itself. This Thesis has been accepted for inclusion in WVU Graduate Theses, Dissertations, and Problem Reports collection by an authorized administrator of The Research Repository @ WVU. For more information, please contact researchrepository@mail.wvu.edu. 


\title{
AN INTEGRATED LATERAL FLOW DEVICE FOR FLOW CONTROL AND BLOOD SEPARATION
}

\author{
Veronica Betancur Calle \\ Thesis submitted to the \\ Benjamin M. Statler College of Engineering and Mineral Resources \\ at West Virginia University \\ in partial fulfillment of the requirements \\ for the degree of \\ Master of Science \\ in \\ Aerospace Engineering \\ Nianqiang $\mathrm{Wu}, \mathrm{Ph} . \mathrm{D}$. , Chairperson \\ Yuxin Liu, Ph.D., Co-Chairperson and Research Advisor \\ Ismail Celik, Ph.D. \\ Department of Mechanical and Aerospace Engineering \\ Morgantown, West Virginia \\ 2016
}

Keywords: passive microfluidics; capillary force; PDMS surface modification, flow control; blood separation; integration of functions; point-of-care

Copyright 2016 Veronica Betancur Calle 


\title{
ABSTRACT An integrated lateral flow device for flow control and blood separation
}

\author{
Veronica Betancur Calle
}

Lateral flow devices (LFD) have been used and are promising in many applications, including point-of-care diagnosis in medical, clinical, food and environmental control and monitoring. In particular, disposable paper-based lateral flow strips utilize low-cost materials and do not require expensive fabrication instruments. Many examples of diagnostic tests have been developed using microfluidics paper analytical devices ( $\mu$ PADs), including tests for the detection of total glucose and protein in urine (colorimetric), detection of $\beta$-galactosidase (fluorescence), and liver enzyme tests for monitoring the health of patients been treated with multiple medications for HIV and/or tuberculosis that can cause hepatotoxicity. However, there are constraints on tuning the flow rate and immunoassays functionalization in paper as well as technical challenge in integration of sensors and concentration units for low-abundant molecular detection. In the present work, we demonstrated an integrated lateral flow device that applied the capillary forces with functionalized polymer-based microfluidics as a strategy to realize a portable, simplified, and self-powered LFD with minimizing the need for off-chip equipment. The LFD combined the ability to separate plasma from human whole blood and create controlled and steady flow using capillary forces. Lateral flow relies on surface tension and hydrophilic natural of the flow channel, and controlled flow is also a key variable for immunoassay-based applications for providing enough time for protein binding to antibodies. To render polydimethylsiloxane (PDMS) surface of hydrophilicity as well as to control the flow rate, PDMS was functionalized with different concentrations of Pluronic F127. The results show that, in an integrated LFD, the flow rate was regulated by the combination of multiple factors, including Pluronic F127 functionalized surface properties of microchannels, resistance of the integrated flow resistor, the dimensions of the microstructures and the spacing between them in the capillary pump, the contact angles, and viscosity of the fluids. The resistance of the flow resistor had the greatest effect on the flow rate of the fluids. Plasma flow rates ranging between 0.43 and $18.60 \mathrm{~nL} / \mathrm{s}$ were achieved in the flow resistor, and between 0.99 and $61.30 \mathrm{~nL} / \mathrm{s}$ in the whole device. In addition, human plasma was separated from whole blood by using a highly asymmetric plasma separation membrane, which captured the cellular components of the blood (red cells, white cells, and platelets), without lysis, so that only high quality plasma could flow down into the smaller pores on the downstream side of the membrane, and into the first capillary pump. The developed LFD can be used as a flexible and versatile platform and has the potential for detecting circulating biomarkers from whole blood; sandwich-immunoassays can be performed directly on the LFD by patterning receptors for analytes on the glass chip, and detection can be performed using a variety of methods ranging from colorimetric and fluorescence methods to more complex methods such as Surface-enhanced Raman spectroscopy (SERS) depending on the limit of detection of the target biomarker. The bio sensing technology being described presents an alternative for point of care testing using small samples of human whole blood; it could benefit regions with limited access to healthcare, where delays in diagnosis can lead to the quick deterioration of the quality of life and increase the morbidity and mortality. 
To my beloved family ... 
"Shoot for the moon...even if you miss, you'll land among the stars! It's better to aim high and miss, rather than to aim low and hit! Get a bigger dream! You have something special. You have GREATNESS within you!" Les Brown 


\section{Acknowledgements}

Though only my name appears on the cover of this thesis, a great many people have contributed to its production. I owe my gratitude to all those people who have made this thesis possible and because of whom my graduate experience has been one that I will cherish forever.

Foremost, I would like to express my sincere gratitude to my adviser Dr. Yuxin Liu, whose continuous guidance, inspiration, and encouragement have been invaluable for my Master's thesis work. It is my extreme privilege to have been a graduate research assistant in her lab for the past two years. I am extremely lucky to have been given the chance of joining her group, and discover a whole new world in the field of microfluidics. I will always remember her as not only my advisor, but also my mentor of life.

I would also like to extend my gratitude to Dr. Nick Wu, whose support, helpful advice, excellent teaching, and many amazing new ideas have been very critical to my work.

I gratefully acknowledge the members of my committee: Dr. Nick Wu, Dr. Yuxin Liu, and Dr. Ismail Celik, for their valuable time and guidance of my research work as well as the valuable feedback on this thesis.

I would like to express my immense gratitude to my fellow lab mates: Jianbo Sun, and Peng Zhang, and all my former lab mates: Dr. Xiang Li, Bhargavi Thotakura, and Hanyuan Zhang; their friendship helped me stay sane through these difficult years. Their support and care helped me overcome setbacks and stay focused on my graduate study. I greatly value their friendship and I deeply appreciate their belief in me. I would like to thank Dr. Xuefei Gao, Dr. Konstantino's Sierros, and Rong Cai for all her help on this research project. 
Moreover, I would like to thank Dr. Kolin Brown, Dr. Weiqiang Ding, Dr. Huiyuan Li, and the SRF staff for their great help with cleanroom-based microfabrication.

I have saved the last word of acknowledgement for my family for all their love, support, and continuous encouragements throughout my years of study and through the process of researching and writing this thesis. This accomplishment would not have been possible without them. To them I dedicate this thesis. 


\section{Table of Contents}

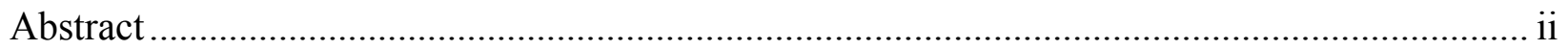

Acknowledgements .......................................................................................................... v

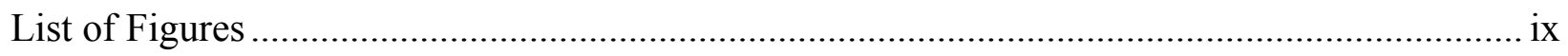

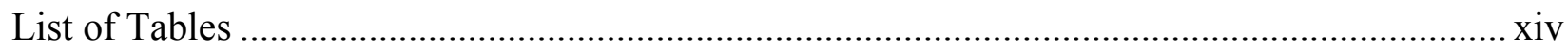

Chapter 1 Thesis Overview.............................................................................................. 1

1.1 The Importance of Strategy Implementation .................................................................. 1

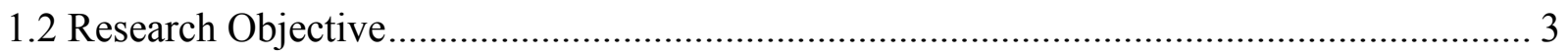

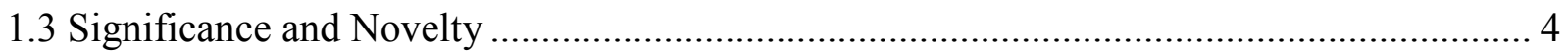

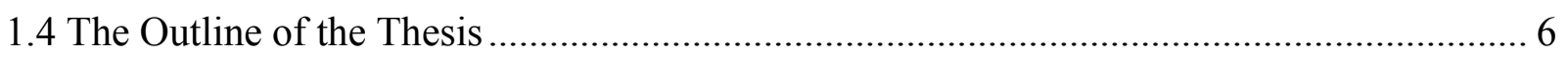

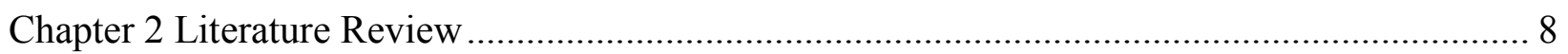

2.1 Microfluidics for point of care immunodiagnostics ........................................................ 8

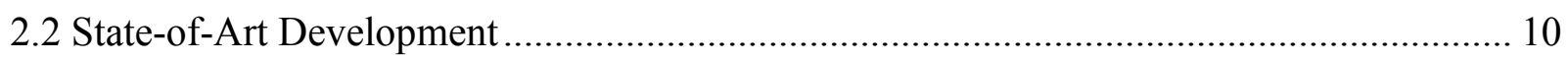

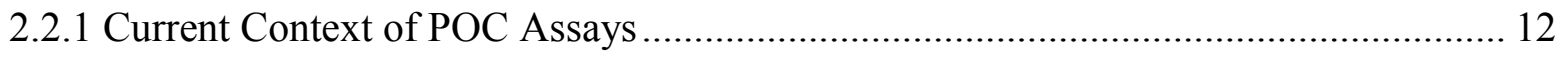

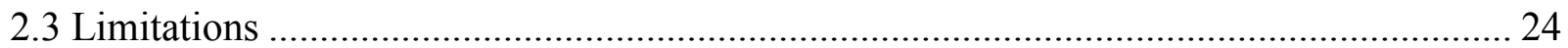

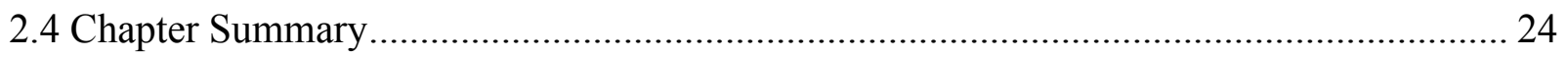

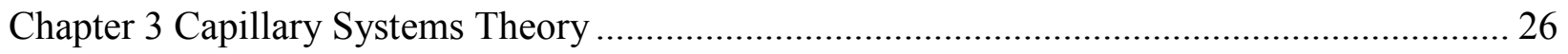

3.1 Capillary Phenomena ………………………………................................................ 26

3.1.1 Wetting and Interfacial Free Energy ...................................................................... 27

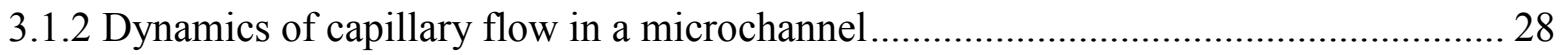

Chapter 4 Capillary Driven Microfluidic Platform................................................................... 35

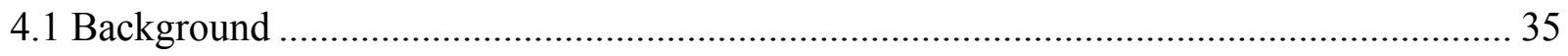

4.2 Design of an Integrated Lateral Flow Device ………..................................................... 36

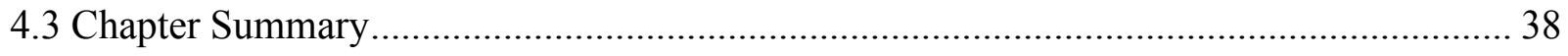

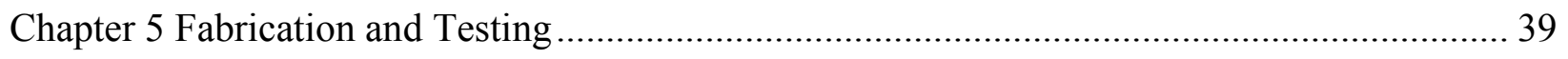

5.1 Fabrication................................................................................................................. 39

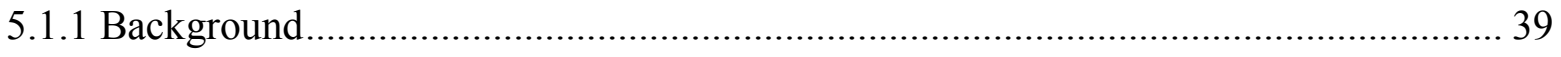

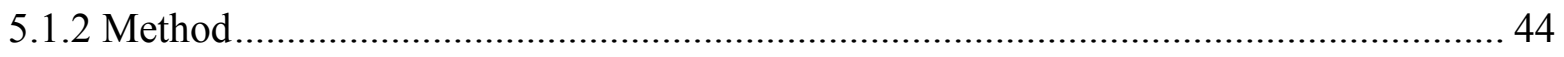

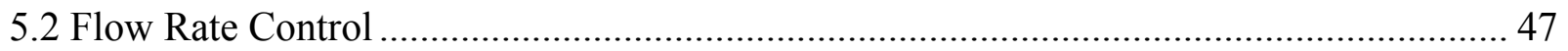

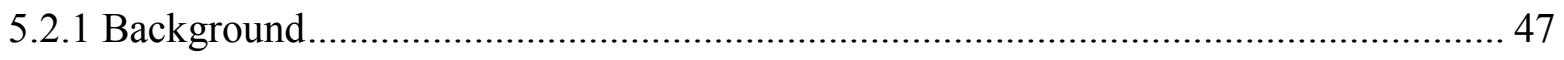

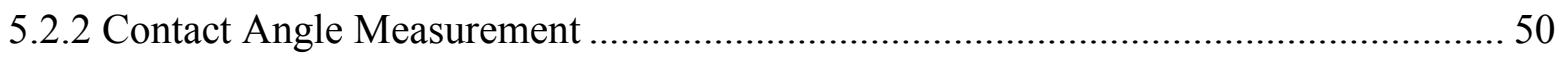




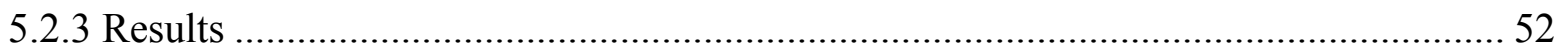

5.3 Evaluation of the Integrated LFD with Human Whole Blood ......................................... 74

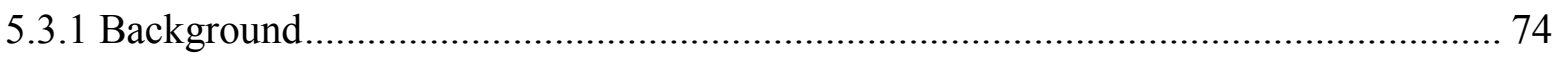

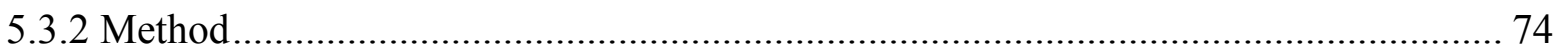

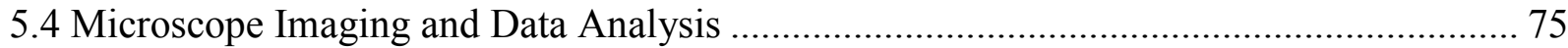

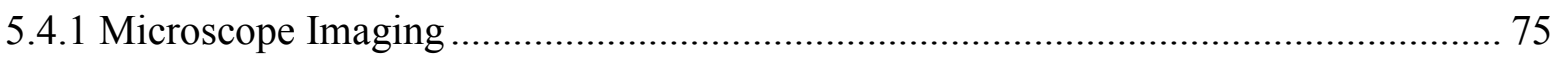

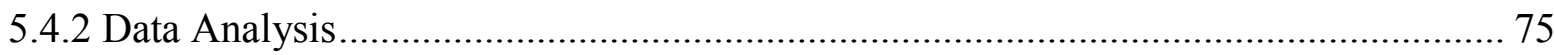

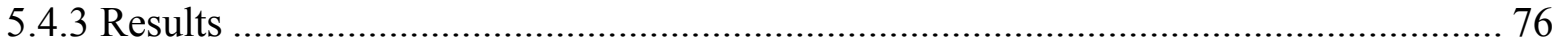

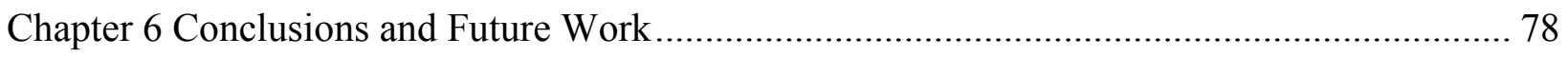

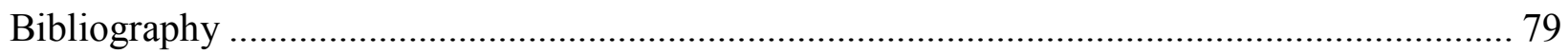




\section{List of Figures}

Figure 2-1 Microfluidic platform developed by Ko-Wei Chang et al. for the quantification of glycated hemoglobin (Hb A1C) using an aptamer-based sandwich assay. (a) A schematic illustration of the trilayer microfluidic chip composed of micro-components, including normallyclosed valves, a transport unit (a closed chamber), a waste chamber and five open chambers; (b) a photograph of the microfluidic chip with dimensions of $28 \mathrm{~mm} \times 30 \mathrm{~mm}$. Note that the red region and blue region represent the liquid layer and the air layer, respectively; (c) an exploded view of the microfluidic chip, which is composed of a thick-film PDMS layer for the air channels, a thin-film PDMS layer for the liquid channels, and a glass substrate; (d) a schematic diagram of five open chambers on the microfluidic chip for loading samples and reagents [40], [41].

Figure 2-2 Design of microfluidic electrochemiluminescence (ECL) array for sensitive measurements of prostate specific antigen (PSA) and interleukin-6 (IL-6) in serum. The different components of the microfluidic platform are identified as following: 1) syringe pump 2) injector valve, 3) switch valve to guide the sample to the desired channel, 4) tubing for inlet, 5) outlet, 6) poly(methylmethacrylate) (PMMA) plate, 7) Pt counter wire, 8) Ag/ $\mathrm{AgCl}$ reference wire (wires are on the underside of PMMA plate), 9) polydimethylsiloxane (PDMS) channels, 10) pyrolytic graphite chip $(\mathrm{PG})(2.5 \mathrm{~cm} \times 2.5 \mathrm{~cm})$ (black), surrounded by hydrophobic polymer (white) to make microwells. Bottoms of microwells (red rectangles) contain primary antibody-decorated SWCNT forests, 11) ECL label containing RuBPY-silica nanoparticles with cognate secondary antibodies are injected to the capture protein analytes previously bound to cognate primary antibodies. ECL is detected with a CCD camera [36].

Figure 2-3 Microfluidic platform developed by Ko-Wei Chang et al. for the quantification of $\mathrm{Hb}$ A1C using a dual aptamer method in lieu of antibodies. (a) Schematic illustration of the chip and its respective components, including six normally-closed valves, two transport unit (closed chamber), a waste outlet, and six open chambers. (b) Photograph of the $43 \mathrm{~mm} \times 53 \mathrm{~mm}$ microfluidic chip. The red and blue regions represent the liquid and air layers, respectively. (c) Exposed view of the microfluidic chip, which was composed of a thick-film PDMS layer for air control, a thin-film PDMS layer for the liquid channels, and a glass substrate. (d) Schematic diagram of the six open chambers on the microfluidic chip for loading samples and reagents [40].

Figure 2-4 Magnetic bead-based microfluidic chip designed by Fang Lee et al. for the analysis of dengue associated antibodies in human plasma. (a) A photograph of the microfluidic chip, comprising one and two-way micropumps, a four-membrane type micromixer, and an on-chip 
microcoil array. The dimensions of this chip are measured to be $53 \mathrm{~mm} \times 37 \mathrm{~mm}$. (b-1) A schematic diagram of the four-membrane-type micromixer; (b-2)-(b-5) show the motion of the fluids in the micromixer [52].

Figure 2-5 Microfluidic protein chip for an ultrasensitive and multiplexed assay of cancer biomarkers in human serum, in the format of both sandwich and reverse phase immunoassay. (a) Schematic illustration of the microchannel network. (1) First, a PDMS piece was placed in a silyated slide; (2) The solutions were flowed through the channels, and the first layer of protein was immobilized; (3) The first PDMS piece was removed; (4) The other PDMS piece was placed on the substrate with channels perpendicular to the first PDMS piece; (5) Other solutions were flowed; (6) A two dimensional array was formed above the chip. (b) Schematic illustration of a sandwich immunoassay and reverse phase immunoassay based on $\mathrm{CdTe} / \mathrm{CdS}$ quantum dots-IgG fluorescent probes and microfluidic protein chip.

Figure 2-6 The schematic representation of microfluidic paper-based three-dimensional electrochemical device, developed by Wang et al. for the simultaneous diagnosis of two tumor markers: cancer antigen 125 and carcinoembryonic antigen. (A): Wax-patterned paper sheet; (B): the 3D-- $\mu$ PED, (a) paper working zones; (b) paper auxiliary zone. (C): Screen-printed electrodes, (c) carbon working electrodes; (d) Ag/AgCl reference electrode; (e) carbon counter electrode; (f) silver conductive channel and pad; (g) transparent polyethylene terephthalate substrate. (D): After stacking, the paper working zones and the paper auxiliary zone will be aligned to the screen-printed working electrodes, counter and reference electrode, respectively [33].

Figure 2-7 PDMS microfluidic chip coupled with Love Wave sensor. (b) Picture of the PDMS microfluidic chip. (c) Schematic of the circuit for Love Wave sensor measurement. (c) Printed circuit board [55].

Figure 2-8 Modified PDMS microfluidic ELISA device for the multiplexing detection of IL-5, $\mathrm{HBsAg}$, and IgG in human serum. The microfluidic device encompassed several microchannels to guide the flow to a detection well, and then drain out to the waste outlet manifold by overflow, in such a way that backflow of the reacted fluid is eliminated, in order to avoid contamination. (a) Top view, (b) Section view of microfluidic ELISA device [61].

Figure 2-9 Schematic diagram and pictures of microfluidic device, and data on fluid handling of a POC ELISA-like assay for the simultaneous diagnosis of HIV and syphilis. (a) Picture of microfluidic chip. Each chip can accommodate seven samples (one per channel), with molded holes for coupling of reagent-loaded tubes. (b) Scanning electron microscope image of a cross- 
section of microchannels, made of injection-molded plastic. Scale bar, $500 \mu \mathrm{m}$. (c) Transmitted light micrograph of channel meanders. Scale bar, $1 \mathrm{~mm}$. (d) Schematic diagram of passive delivery of multiple reagents, which requires no moving parts on-chip. A preloaded sequence of reagents passes over a series of four detection zones, each characterized by dense meanders coated with capture proteins, before exiting the chip to a disposable syringe used to generate a vacuum for fluid actuation. (e) Illustration of biochemical reactions in detection zones at different immunoassay steps. The reduction of silver ions on gold nanoparticle-conjugated antibodies yields signals that can be read with low-cost optics (for quantification) or examined by eye. (f) Absorbance traces of a complete HIV-syphilis duplex test as reagent plugs pass through detection zones. High optical density (OD) is observed when air spacers pass through the detection zones, owing to increased refraction of light compared to in the liquid filled channels. The train of reagents mimics the pipetting of reagents in and out of multiwall plates. This sample was evaluated (correctly against a reference standard) as HIV negative and syphilis positive. Ag, antigen [62]

Figure 2-10 Nanobiosensor based on localized surface plasmon resonance (LSPR) for the detection of microalbuminuria. The principle (left) and technologies developed (right) for forming the LSPR POC system [37]

Figure 3-1 (a) Surface tension forces at the three-phase line on a non-deformable solid surface [97].

Figure 4-1 A) Schematic diagram of lateral flow device (LFD) for detection of single biomarker in whole blood sample. The first capillary pump (CP1) is partially covered by a GR plasma separation membrane, and it is designed for plasma extraction, while the second capillary pump (CP2) is designed to assist the fluid flow. Surface-immunoassays can be performed directly on the sealed chip by patterning capture antibodies on the gold chip placed on top of the detection chamber area. B) Schematic of assembled LFD. The black arrows indicate the flow direction. . 37

Figure 5-1 Microfluidics are fabricated in a variety of materials, including glass, silicon, paper, elastomers like PDMS, hydrogels, thermoplastics, and thermosets. Fabrication processes differ in degrees of complexity, and therefore in cost. The selection of the material to be used will depend on several factors, including the application, the target audience, and the fabrication capabilities 
Figure 5-3 Representative images showing the change of the static contact angles of a $10 \mu \mathrm{L}$ droplet of plasma Pluronic functionalized PDMS $[4 \mu \mathrm{L} / \mathrm{g}]$. A) Initial contact angle just after dispensing the droplet. B) 5 min after dispensing the droplet. C) $10 \mathrm{~min}$ after dispensing the droplet. D) 15 min after dispensing the droplet. E) 20 min after dispensing the droplet.

Figure 5-4 A) Schematic of Dynamic contact angle in Flow Resistors. The interface has concave contact lines for the side walls and nearly flat $\left(90^{\circ}\right)$ contact lines for the top and bottom walls; the contact lines with the top and bottom walls can also have a convex shape when the contact angle is greater than $90^{\circ}$. The light area represents the plasma phase, which moves from left to right. B) Hydrophillic channel (Pluronic functionalized PDMS (Pluronic/PDMS [4 $\mu \mathrm{L} / \mathrm{g}]$ ). C) Hydrophobic channel (Pluronic functionalized PDMS (Pluronic/PDMS $[2 \mu \mathrm{L} / \mathrm{g}]$ ). The black arrows indicated the flow direction in the microchannel.

Figure 5-5 Representative results showing the dynamic contact angles in the capillary pumps. A) CP1, Plasma, Pluronic functionalized PDMS (Pluronic/PDMS volume ratio: 1:259). B) CP2, plasma, Pluronic functionalized PDMS (Pluronic/PDMS volume ratio: 1:259). C) CP1, PBS, Pluronic functionalized PDMS (Pluronic/PDMS volume ratio: 1:518). D) CP2, Plasma, Pluronic functionalized PDMS (Pluronic/PDMS volume ratio: 1:518). The black arrows indicated the flow direction in the capillary pumps.

Figure 5-6 Dynamic contact angle in each functional unit of the device using a Pluronic/PDMS concentration of $[2 \mu \mathrm{L} / \mathrm{g}]$

Figure 5-7 Dynamic contact angle in each functional unit of the device using a Pluronic/PDMS concentration of $[4 \mu \mathrm{L} / \mathrm{g}]$

Figure 5-8 Relationship between the geometric resistance variation and the flow rate of plasma.

Figure 5-9 Flow rate in first capillary pump

Figure 5-10 Flow rate of secondary capillary pump

Figure 5-11 Schematic of process of surface modification. A) Before water treatment. B) Water treatment by full immersion. C) Water treatment by partial immersion... 
Figure 5-12 Contact angle of water treated (partially immersed) LFD at the flow resistor. The concentration of pluronic was $4 \mu \mathrm{L} / \mathrm{g}$. The black arrow indicates the direction of the flow. 66

Figure 5-13 Flow rate of the entire device. A) Pluronic Functionalized PDMS [2 $\mu \mathrm{L} / \mathrm{g}$ ] without water treartment. B) Pluronic Functionalized PDMS [4 $\mu \mathrm{L} / \mathrm{g}]$ without water treartment. C) Pluronic Functionalized PDMS [2 $\mu \mathrm{L} / \mathrm{g}$ ] with water treartment. D) Pluronic Functionalized PDMS $[4 \mu \mathrm{L} / \mathrm{g}]$ with water treartment.

Figure 5-14 Comparison of static contact angle with and without water treatment 70

Figure 5-15 Comparison of dynamic contact angle of water treated devices based on the immersion mode.

Figure 5-16 Comparison of the experimentally measured and the theoretically calculated flow rate using the static contact angles.

Figure 5-17 Plasma separation from whole blood. A) Ready to separate. B) Start to separate. C) Continuous separation. The black arrows indicate the direction of the flow. 76

Figure 6-1 Schematic of potential design for a multiplexing platform. 78 


\section{List of Tables}

Table 2-1 Summary of biomarker detection using microfluidic platforms [38] ......................... 23 Table 5-1 Summary of the reported detection methods, example analytes and applications for both paper-based microfluidic devices and paper-based microarray plates [129]....................... 42

Table 5-2 Total flow-rate resistance vs. Hydraulic resistance PBS ............................................ 51

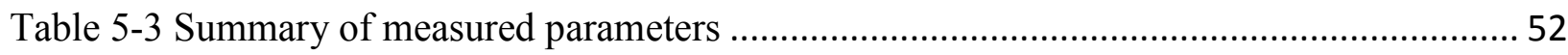

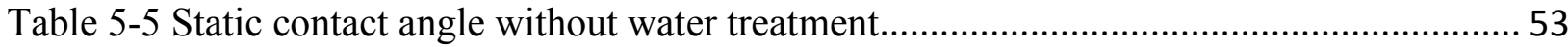

Table 5-5 Dynamic contact angle in flow resistor (Top/Bottom) ............................................... 57

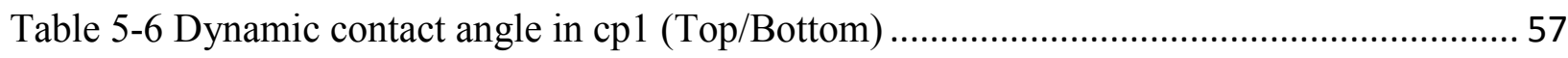

Table 5-7 Dynamic contact angle in cp2 (Top/Bottom) ………............................................. 58

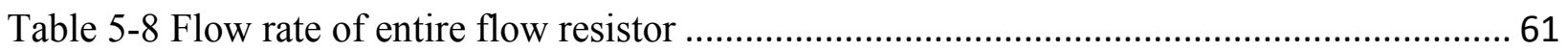

Table 5-9 Decay of flow rate across the flow resistor (Pluronic/PDMS $[4 \mu \mathrm{L} / \mathrm{g}]$ ) …................... 62

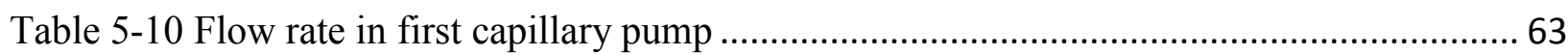

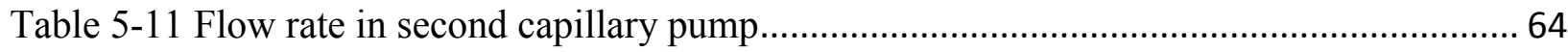

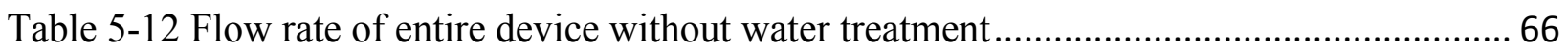

Table 5-13 Flow rate of entire device with water treatment ................................................. 67

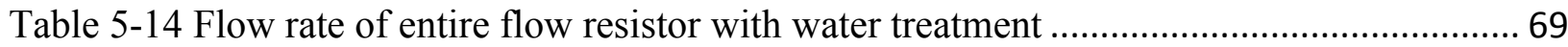

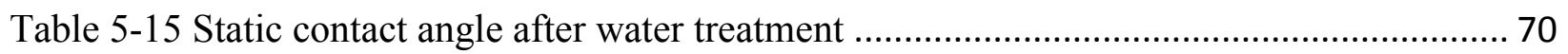

Table 5-16 Dynamic contact angle of flow resistor after water treatment (full immersion) ......... 71 Table 5-17 Dynamic contact of flow resistor after water treatment (patterned face in contact with

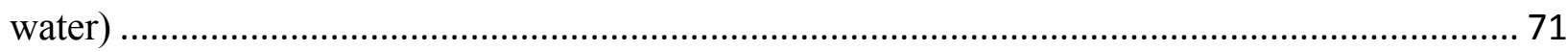

Table 5-18 Validation data (Plasma) …………………………………………………. 73

Table 5-18 Comparison of flow rates of the flow resistor (entrance) for separated plasma from whole blood and just plasma. The substrate used was Pluronic functionalized PDMS

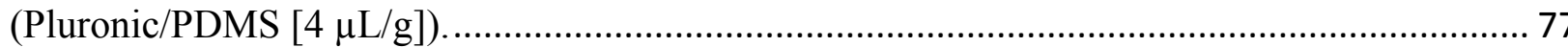




\section{Chapter 1 Thesis Overview}

\subsection{The Importance of Strategy Implementation}

Because financial resources are limited in developing countries, their habitants tend to have less access to proper health services as a result of the high cost of current detection and monitoring methods, which are often time consuming and do not provide immediate results, delaying the process of targeting, and controlling the disease in order to prevent its worsening [1]. The delay in diagnosis, limitation in monitoring capabilities, and obstruction of the prevention of risk factors will lead to the deterioration of the quality of life of the patients and increase the morbidity and mortality in these areas. The outcome is a vicious cycle where poverty leads to the decrease in health quality, and the decrease in health quality results in further socioeconomic deterioration.

Proper and on time intervention could change the clinical outcome of the patients by decreasing the negative impact in the quality of life by avoiding the functional deterioration left by the aftermath and reducing the comorbidities and cost of treatment, the number of required hospitalizations, and the mortality rate. With this in mind, there is an increasing need of methods that allow assessing health status, disease onset and progression, and monitoring treatment outcome for the study of these patients [2]. These methods must be user friendly, cost efficient, reliable, portable, and generate fast results.

The second half of the 20th century has brought great advances in biochemistry and technology, leading to greater sensitivity and more specific analysis of samples [3]. A manifestation of this potential is in the emergence of microfluidic bioanalysis. The science of Microfluidics concerns the controlled transport of low volumes of fluid, and it is the key for 
miniaturization, as well as the integration of multiple functionalities, allowing for the performance of complete laboratory protocols on a single chip [4], [5]. Performing analytical and diagnostic tasks in microfluidic-based systems presents several advantages, including small sample volume requirements, rapid transport times and portability, as well as allowing for continuous flow with high precision, greatly improving the efficiency and reliability of devices for point-of-care diagnostics [6]-[8]. Ideally, a microfluidic system should self-contained, simple to use, flexible, robust, and its fabrication should be uncomplicated [9]. Low cost is important because it allows delivering the technologies to a wider population. Sensitivity leads to more reliable tests, reducing the possibilities of obtaining false positives or false negatives. As users might come from diverse backgrounds and might not have a deep understanding of the working mechanism, these devices must be easily operated and interpreted. These technologies could aid reach many people in need, not only through point-of-care but also through prevention public health brigades for marginalized and captive populations.

Unfortunately, the application of such systems in home and point-of-care situations has been limited because they typically require external macroscopic actuators, bulky fluidic connections, and electromechanical interfaces to initiate and control fluid flow [6], [9]-[12]. Passive flow methods are an alternative in overcoming this limitation, because they demand no external power input, making them quite compact and thus portable; resulting in attractive analytical tools for applications such as single use assays and simple diagnostics [6]. Capillarybased pumping takes advantage of surface tension to pull fluid through the device, so the fluid needs only to be delivered to an inlet region (usually via pipette); the spontaneous filling of the microfluidic network $(\mu \mathrm{FN})$ which follows, and the subsequent continuous flow are function of the device's design [6], [8]. 


\subsection{Research Objective}

The main objective of this research is to develop a functionalized polymer based lateral flow device (LFD) with integration of function units for studying the capillary flow and controlling the flow rate, with the ultimate goal for the assessment of health status, disease onset and progression, and monitoring treatment outcome. Biomarkers are measurable biological characteristics that can serve as indicators of normal biological processes, pathogenic processes, pharmacologic responses to therapeutic intervention, and the risk or the presence of a disease. The objective measurement and evaluation of circulating biomarkers can therefore help in the assessment of the biological state of a person.

The developed platform should be able to separate plasma from human whole blood, create steady flows and control the rate of flow by using passive microfluidics, and have the potential for detecting circulating biomarkers from the separated plasma. Specifically, the research focuses on:

1) Development of a microfluidic platform that operates under capillary forces to initiate and control the fluid flow. Capillary action was achieved by two means: the use of capillary pumps, and the surface functionalization of the polydimethylsiloxane (PDMS) using Pluronic F127. Capillary pumps (CPs) comprise microstructures of diverse shapes, with dimensions in the micrometer range, whose position in the CPs encodes the desired capillary pressure. Because PDMS has a hydrophobic nature, its surface was functionalized with Pluronic F127 to render it hydrophilic.

2) Develop strategies to control the rate of flow, which would allow for providing enough time for protein binding to antibodies and biomarker detection in the future. The binding reaction 
between the antigen and the detection and capture antibodies takes a determined amount of time to reach equilibrium, making the time available for binding a critical factor in the optimization of an immunoassay. The flow rate was regulated by the combination of multiple factors, including Pluronic F127 functionalized surface properties of microchannels, resistance of the integrated flow resistor, the dimensions of the microstructures in the capillary pumps and the spacing between them, contact angles, and viscosity of the fluids.

3) Integration of plasma separation membrane for performing in-situ plasma separation from human whole blood. The highly asymmetric nature of a plasma separation membrane allows the cellular components of the blood (red cells, white cells, and platelets) to be captured in the larger pores without lysis, while the plasma flows down into the smaller pores on the downstream side of the membrane.

4) The method for sensor chip integration which can provide a convenient and easy way to work with different sensing approaches, including but not limited to colorimetry and fluorescence. This large degree of flexibility will allow for performing different types of immunoassays.

\subsection{Significance and Novelty}

Commonly used methods for quantification of protein markers in clinical setting are immunonephelometry or enzyme-linked immunosorbent assay (ELISA). Both require a centralized lab to separate whole blood and to perform multiple strict washing steps over several hours. There is a strong incentive to develop a rapid point-of-care (POC) device for protein biomarker detections with improved diagnostic modalities, enable of using minimal amounts of blood, and capable of returning a rapid result. 
In an attempt to address this necessity, several microfluidic devices have been fabricated in the past with the goal of performing only a single task on a microliter volume of sample [13]. However, would it not be more effective and practical to combine the functions of different such devices on an integrated microfluidic chip, instead of having to perform each task separately on different devices? Although the degree of complexity increases with the integration, integrated microfluidic devices offer several advantages, including a higher degree of accuracy, contamination control, ease of use, rapid results, and low cost [13]. It is much easier to utilize a single integrated device rather than using several chips, not to mention that integrated systems can accurately control the amount of reaction agents and synchronize the reaction time in different reactors [13].

The research work in this thesis demonstrated an integrated passive microfluidic pumping mechanism as an alternative noninvasive monitoring tool for clinical diagnostics requiring only 30-40 $\mu \mathrm{L}$ of blood. Blood was selected as the working fluid as various disease-signaling biomarkers can be found in it, accurately reflecting normal and disease states in humans. This platform combines the ability to perform plasma separation from human whole blood, control the flow rate, integrate the detection sensor, and perform biomarker sensing all in one chip, which shortens the time of sample processing as well as allowing for rapid detection. It further eliminates the need for blood sample pretreatment in a centralized laboratory prior to the detection. The flow in the lateral flow device is powered by the capillary force, which was accomplished by two means: the use of capillary pumps (CPs) [11], and the surface functionalization of the PDMS using Pluronic F127 [12], thus eliminating the need of external power and reducing the cost. 
In order to extend the performance of capillary systems (CSs), we designed, fabricated and tested a range of CSs, and compared their experimental filling behavior. The CSs were fabricated on polydimethylsiloxane (PDMS) using soft lithography, and sealed with a blank piece of PDMS. Because PDMS is of hydrophobic nature, it suffers serious nonspecific protein adsorption, therefore requiring of surface modification strategies [12]. Nonionic surfactants can adsorb strongly on hydrophobic surfaces rendering them hydrophilic and nonionic, preventing the interaction between proteins and the surface [12], with this in mind, PDMS surface was functionalized with Pluronic F127 to render it hydrophilic. The concentration of Pluronic was optimized for the sake of controlling the flow rate, allowing performing reliable sandwich immunoassays for biomarker detection. In addition, the Pluronic F127-modified PDMS has been shown to significantly suppress the non-specific adsorption of proteins due to the improved hydrophilicity, compared to native PDMS [14], [15].

The promise of this novel LFD lies in its potential for point of care applications, offering an integrated and flexible platform, which can perform full laboratory procedures for the rapid and proper diagnosis in one single chip.

\subsection{The Outline of the Thesis}

This thesis consists of six chapters. It is initiated with the thesis overview in Chapter One, in which the importance of strategy implementation, research objectives, significance, and novelty of this research were stated. Following chapter one, six chapters introduced the details of the research work. Chapter Two is an introduction to the microfluidics field, including its history, and the state-of-art of point of care devices for health applications. Chapter Three described the theory behind capillary phenomena. Chapter Four introduced the design of the 
integrated lateral flow device, demonstrated fabrication procedures and testing methodology. Chapter Five characterized the flow rate control with the combined analysis of static/dynamic contact angles, functionalization of PDMS, and flow resistance, as well as the blood separation capabilities. Conclusions and future directions are summarized in Chapter Six. 


\section{Chapter 2 Literature Review}

\subsection{Microfluidics for point of care immunodiagnostics}

The second half of the 20th century has brought great advances in biochemistry and technology, leading to greater sensitivity and more specific analysis of samples [3]. A manifestation of this potential is in the emergence of microfluidic bioanalysis.

The science of Microfluidics concerns the controlled transport of low volumes of fluid, and it is the key for miniaturization, as well as the integration of multiple functionalities, allowing for the performance of complete laboratory protocols on a single chip [4], [5]. Microfluidics are versatile, and serve a wide variety of purposes such as medical, agriculture, environmental (e.g. toxicity detection), and military applications.

The most promising opportunities of microfluidics for diagnostics might reside in point of care (POC) applications due to their portability, short sample processing time, small sample requirements, ease of use, multiplexing capability, flexibility, and their ability to carry out separations and detections with high resolution and sensitivity [3], [4], [16], [17]. Thanks to these technologies, the process of targeting and controlling diseases in order to prevent their worsening can be performed much earlier, providing patients with a much better quality of life, and increasing the probabilities of a favorable outcome. However, the application of such systems in home and point-of-care situations has been limited because they typically rely on external macroscopic actuators, bulky fluidic connections, and electromechanical interfaces to initiate and control fluid flow [4], [6], [9]-[12]. Capillary phenomena can serve as a substitute to these external equipment in view of autonomously transporting these small volumes of fluid for fueling miniature scaled bio/chemical processes [4]. 
We owe the existence of microfluidics to four main motivational forces: molecular analysis, biodefence, molecular biology and microelectronics [16]. First came analysis. Microanalytical methods such as gas-phase chromatography (GPC), high-pressure liquid chromatography (HPLC) and capillary electrophoresis (CE) allowed for manipulating liquids at high precision, and when combined with the power of the laser for optical detection, they made it possible to achieve high sensitivity and high resolution with low sample volumes [3], [16]. But these methods needed yet to become more compact and versatile [16].

Second, with the cold war came the need of counteracting the threat of chemical and biological weapons [16]. The Defense Advanced Research Projects Agency (DARPA) supported a series of programs for the development of field-deployable microfluidic systems which could detect these threats [16]. This greatly stimulated for the rapid growth of academic microfluidic technology [16].

Third, came the pushing force of molecular biology [16]. With the 1980s explosion of genomics, as well as the advent of other areas of microanalysis related to molecular biology, came the need analytical methods with much greater throughput, which could achieve higher sensitivity and resolution [16].

Fourth came the contribution of microelectronics [16]. The concept of microelectronics started around 60 years ago [4]; it inspired the ideal of miniaturization along with integration and automatization for providing parallelism, enhanced speed, portability, and reduced consumption of sample [4]. In microfluidics, electrons are conceptually replaced by the innumerable amount of chemical species, and the electrical wires by miniaturized pipes [4]. Another great 
contribution from the microelectronics is the development of technologies such as photolithography [4].

The interest of the academic research community in microfabricated microfluidic systems started in the mid-eighties, and has been growing steadily since then [4]. Some of the first work in microfluidics used silicon and glass, but neither of them where the most appropriate for the analysis of biological samples [16]. Elastomers such as poly(dimethylsiloxane) allow for a much easier fabrication process [16].

The appearance of micropumps and valves was consequence of the need of a more precise control of liquid for advanced analysis applications [3], [18]-[20]. Pioneer work on miniaturized total chemical analysis systems ( $\mu$ TAS) was done by Manz et al. (1990) and led to the development of microfluidic high-pressure liquid chromatography systems as well as microfluidic capillary electrophoresis (1992) [3], [21]-[23].

Following research in academia, early microfluidic life science companies such as Caliper Life Sciences (1995), and Cepheid (1996) appeared, and Hewlett Packard started producing gas chromatography, capillary electrophoresis, and high-pressure liquid chromatography systems for laboratories and miniaturizing analytical equipment [3]; these companies were pioneers in the commercialization of these types of systems [3].

\subsection{State-of-Art Development}

The ability to understand and diagnose disease has experienced a great progress over the past decades due to the advances in the quantification of molecular biomarkers [24]. Techniques such as genetic sequencing, mass spectrometry, flow cytometry, and nuclear magnetic resonance have allowed for higher sensitivity, promising improvements in early diagnosis, accurate 
monitoring of existing diseases, and understanding of the underlying causes and mechanisms of disease. However, these techniques are highly complex, and therefore expensive, and they require special infrastructure, and a high degree of training [24]. POC testing is one of the most promising applications of microfluidics, since they can be performed by a clinician, or directly by the patient, without the need of a clinical lab, and with easy handling [24], [25]. Key requirements for a POC device include its ease of use, robustness of reagents and consumables in storage and usage, results concordant with established laboratory methods, and being safe to use [23]. The degree of integration of a microfluidic technology can vary from having disposable microfluidic chips that work in conjunction with peripheral equipment (active microfluidic systems), to having all functions needed for flow initiation and control, sample processing and analysis, and reporting the results on a chip (passive based microfluidic systems) [25].

POC diagnostics have been available for many years, and have proven very beneficial [24]; their success has motivated the research and development of more complex chips where entire laboratory procedures can be performed [24]. The earliest POC tests focused on glucose detection, and they were based on tablets containing the test reagents [26]. Technological advances led to the development of dipstick devices, which evolved in time to self-contained LFD (e.g., for the detection of pregnancy, cardiac disease, and HIV-1 biomarkers) [26]. After years of evolution, current POC devices combine advances in electronics, photonics, microelectromechanical systems (MEMS), and microfluidics to build small, low cost chips that have a unique capability to perform full laboratory procedures in a portable device (lab-on-a-chip) [24], [26]. Microfluidic devices for POC applications differ from conventional microfluidics in their simplicity of use, and little or no use auxiliary external equipment [26].

In what follows some of the advances in the field of POC for health applications are discussed. 


\subsubsection{Current Context of POC Assays}

A number of clinical specimens (e.g., whole blood, sera, saliva, urine, and cerebrospinal fluid) contain free-floating biomarkers that can be used for the diagnosis and monitoring of diseases [27], [28]. Immunoassays are one of the most widely used biomarker sensing techniques that are adapted for point-of-care microfluidic-based diagnostics [24], [29]. Current biosensor formats aimed at disease biomarker detection use some form of immunoassay [29]. Immunoassays rely on the binding interactions between antigens and antigen specific antibodies to detect biological markers from either pathogen or host immune responses [24], [29]. The monomer antibodies used for immunoassay purposes possess ' $\mathrm{Y}$ ' shaped structure, which contain two antigen binding sites [29]. The recognition site of a target antigen by a specific antibody binding site is called epitope, and even though antigens contain numerous epitopes, the binding of an antibody to a unique epitope is highly specific, making immunoassays a highly repeatable and highly specific reaction format [29]. Target biomarkers span the entire range of pathogen types, from viruses (e.g., HIV, Avian Influenza, Papillomavirus, Dengue, and Hepatitis) to bacteria (e.g., tuberculosis) and parasites (e.g., African trypanosomiasis, malaria). Immunoassays are also commonly used for detection of noncommunicable diseases (e.g., cardiovascular diseases, cancer [30]-[37], diabetes) [24].

POC microfluidic platforms have been manufactured using a wide range of materials, including glass, polydimethylsiloxane (PDMS), poly(methyl methacrylate) (PMMA), poly(cyclic olefin), paper, and combinations of the previous [38].

\subsubsection{Point of Care Chemiluminescent Biosensors}


In chemiluminescence, the emission of light is triggered by a chemical reaction resulting from target binding [39]. This methodology is convenient for POC application because it doesn't require excitation light sources and emission filters (which is the case for fluorescence based methods) [39]. Chemiluminescent microfluidic biosensors have been used for a variety of applications, including the quantification of glycated hemoglobin (Figure 2-1) [40]-[43], and the study of biomarkers for the diagnosis of Hepatocellular Carcinoma [44] and treatment monitoring of Leukemia [45].
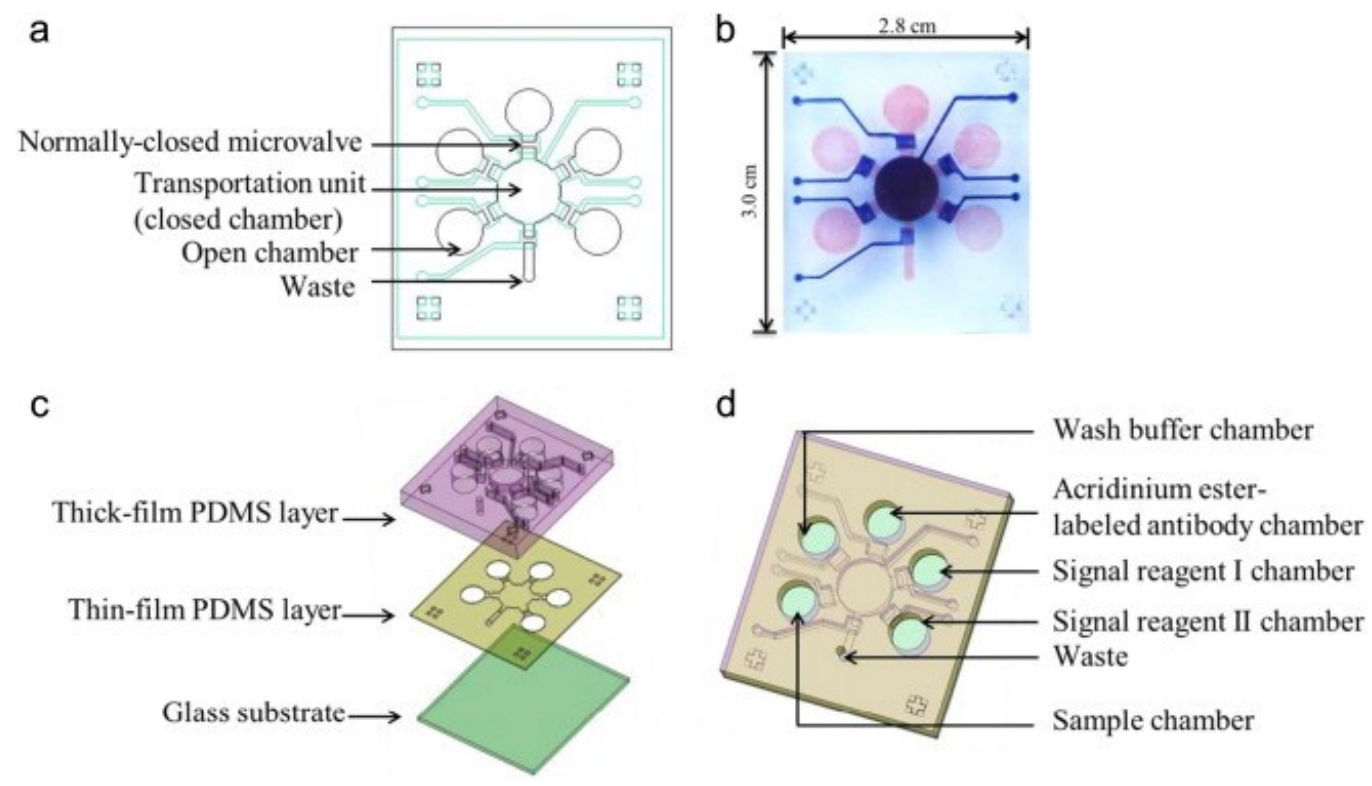

Figure 2-1 Microfluidic platform developed by Ko-Wei Chang et al. for the quantification of glycated hemoglobin ( $\mathrm{Hb} \mathrm{A1C}$ ) using an aptamer-based sandwich assay. (a) A schematic illustration of the trilayer microfluidic chip composed of micro-components, including normallyclosed valves, a transport unit (a closed chamber), a waste chamber and five open chambers; (b) a photograph of the microfluidic chip with dimensions of $28 \mathrm{~mm} \times 30 \mathrm{~mm}$. Note that the red region and blue region represent the liquid layer and the air layer, respectively; (c) an exploded view of the microfluidic chip, which is composed of a thick-film PDMS layer for the air channels, a thin-film PDMS layer for the liquid channels, and a glass substrate; (d) a schematic diagram of five open chambers on the microfluidic chip for loading samples and reagents [40], [41].

\subsubsection{Point of Care Electrochemiluminescent (ECL) Biosensors}


In electrochemiluminiscence (ECL), luminescence occurs as result of electrochemical reactions triggered and controlled by a set of electrodes [39]. ECL has several advantages for POC application, including its versatility and simple optical setup (compared to photoluminescence), its good temporal and spatial control (compared with chemiluminescence), and its negligible background signal [39]. One of the applications of ECL microfluidic biosensors is the study different types of cancer, including ovarian cancer [35], prostate cancer (Figure 2-2) [36], and Colorectal Carcinoma [34].

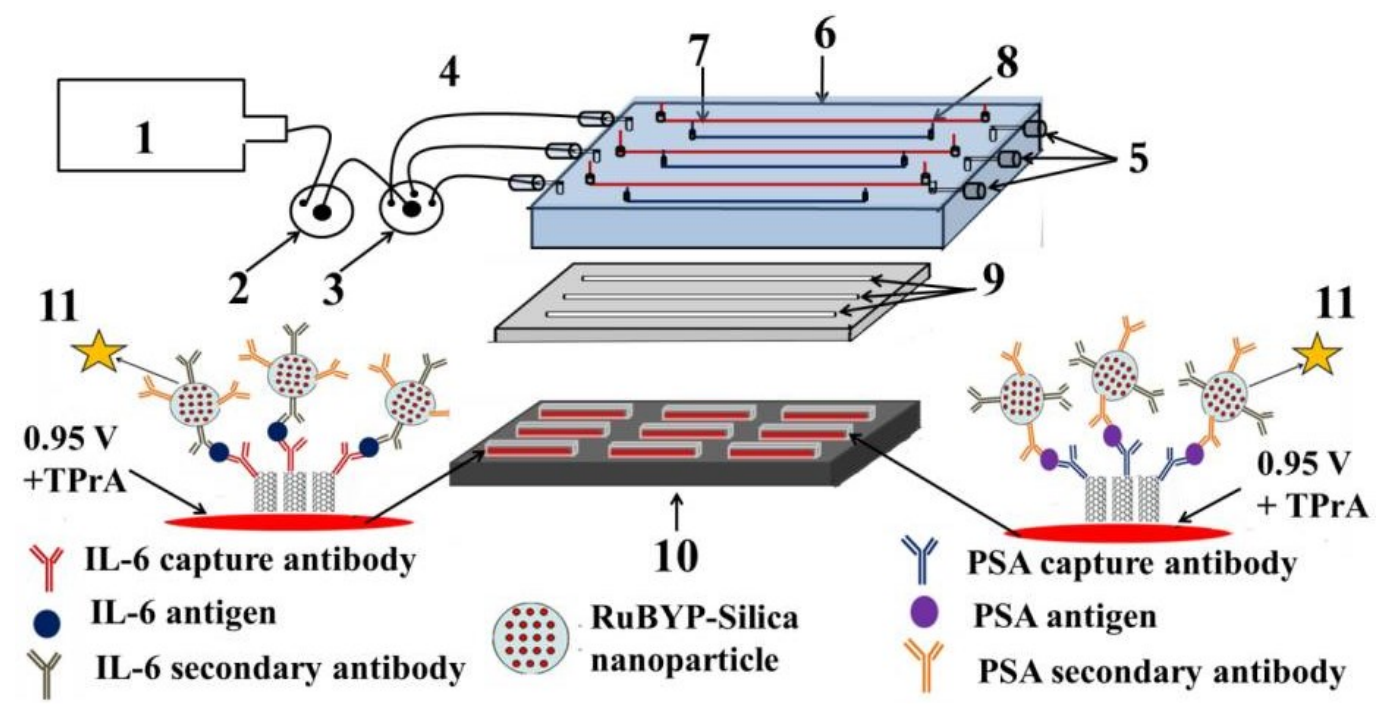

Figure 2-2 Design of microfluidic electrochemiluminescence (ECL) array for sensitive measurements of prostate specific antigen (PSA) and interleukin-6 (IL-6) in serum. The different components of the microfluidic platform are identified as following: 1) syringe pump 2) injector valve, 3) switch valve to guide the sample to the desired channel, 4) tubing for inlet, 5) outlet, 6) poly(methylmethacrylate) (PMMA) plate, 7) Pt counter wire, 8) $\mathrm{Ag} / \mathrm{AgCl}$ reference wire (wires are on the underside of PMMA plate), 9) polydimethylsiloxane (PDMS) channels, 10) pyrolytic graphite chip $(\mathrm{PG})(2.5 \mathrm{~cm} \times 2.5 \mathrm{~cm})$ (black), surrounded by hydrophobic polymer (white) to make microwells. Bottoms of microwells (red rectangles) contain primary antibody-decorated SWCNT forests, 11) ECL label containing RuBPY-silica nanoparticles with cognate secondary antibodies are injected to the capture protein analytes previously bound to cognate primary antibodies. ECL is detected with a CCD camera [36].

\subsubsection{Point of Care Fluorescence-based Biosensors}


Fluorescence remains one of the most widely used methods for detection thanks to the availability of highly sensitive and selective fluorescence labeling techniques [39]. Fluorescent dyes are composed of small molecules, proteins, or quantum dots, which emit a photon when exited [39]. The limitation of flurescent methods lies in its need of more complex optical detection systems compared to colorimetric methods for example [39]. Fluorescence-based microfluidic biosensors have been used in applycations such as glycated hemoglobin quantification (Figure 2-3) [40], point-of-care testing in case of an exposure/intoxication to biological toxins [46], recognition of dengue virus (Figure 2-4) [46]-[48], detection of Neisseria meningitidis bacteria [49], detection of avian influenza H5N1 virus [50], and assessment of colorectal carcinoma biomarkers (Figure 2-5) [51].

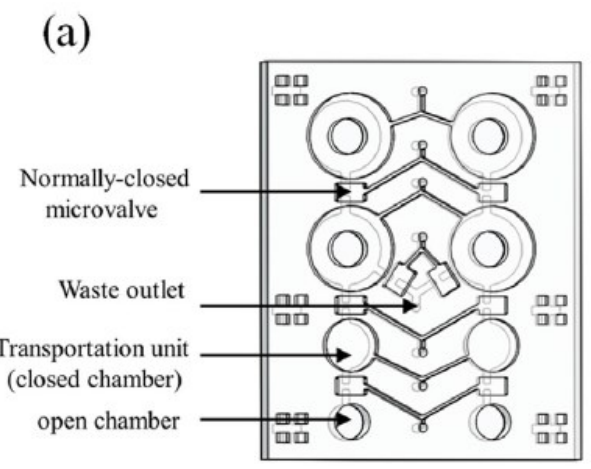

(b)
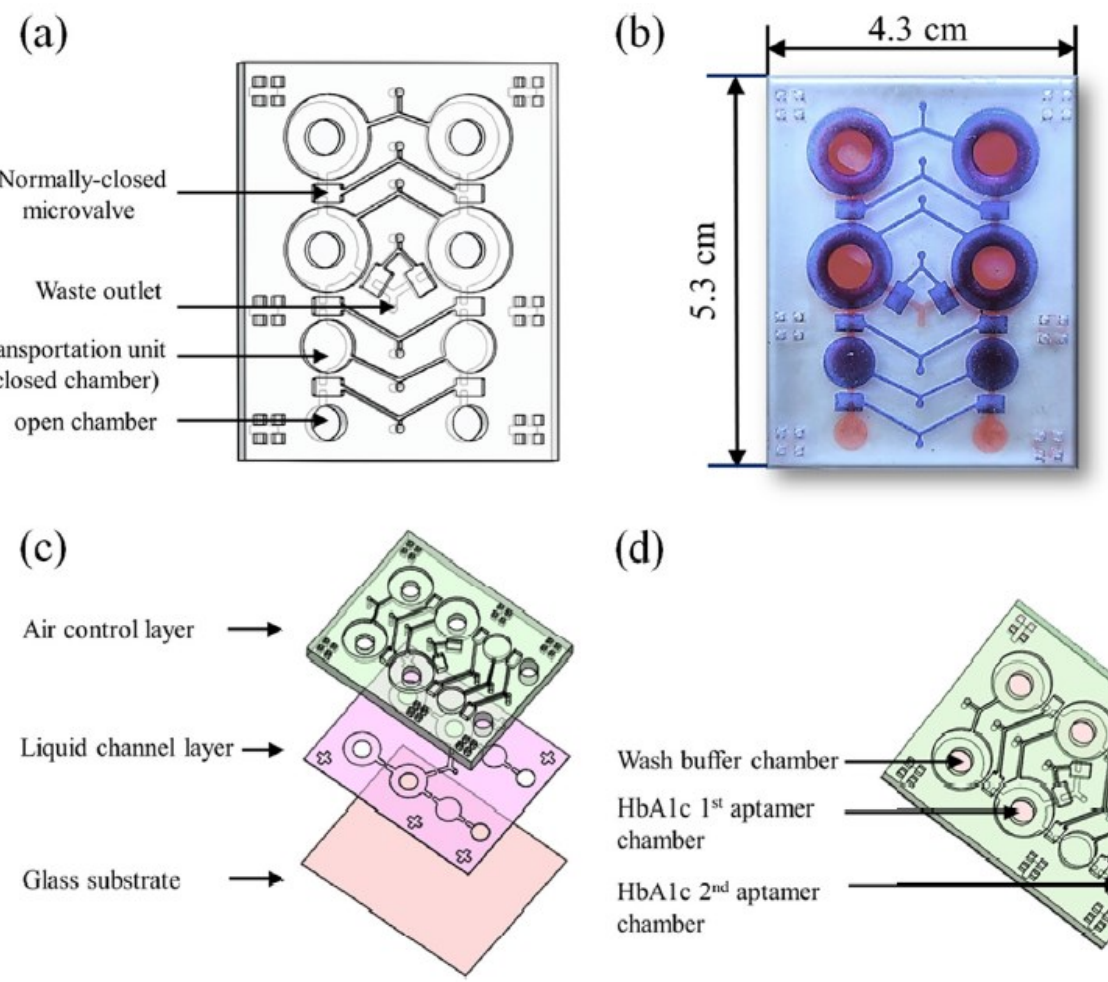

(d)

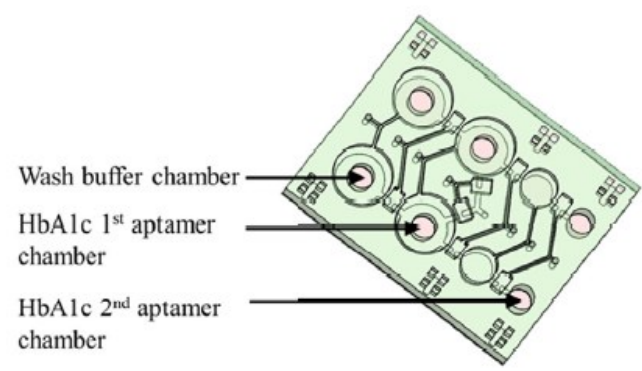

Figure 2-3 Microfluidic platform developed by Ko-Wei Chang et al. for the quantification of $\mathrm{Hb}$ A1C using a dual aptamer method in lieu of antibodies. (a) Schematic illustration of the chip and its respective components, including six normally-closed valves, two transport unit (closed chamber), a waste outlet, and six open chambers. (b) Photograph of the $43 \mathrm{~mm} \times 53 \mathrm{~mm}$ 
microfluidic chip. The red and blue regions represent the liquid and air layers, respectively. (c) Exposed view of the microfluidic chip, which was composed of a thick-film PDMS layer for air control, a thin-film PDMS layer for the liquid channels, and a glass substrate. (d) Schematic diagram of the six open chambers on the microfluidic chip for loading samples and reagents [40].

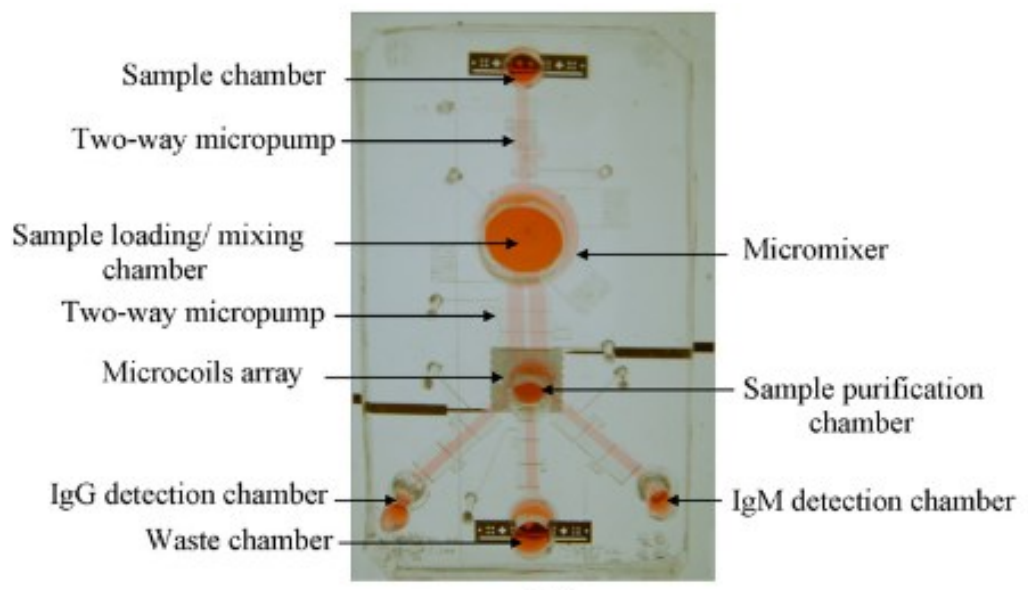

(a)
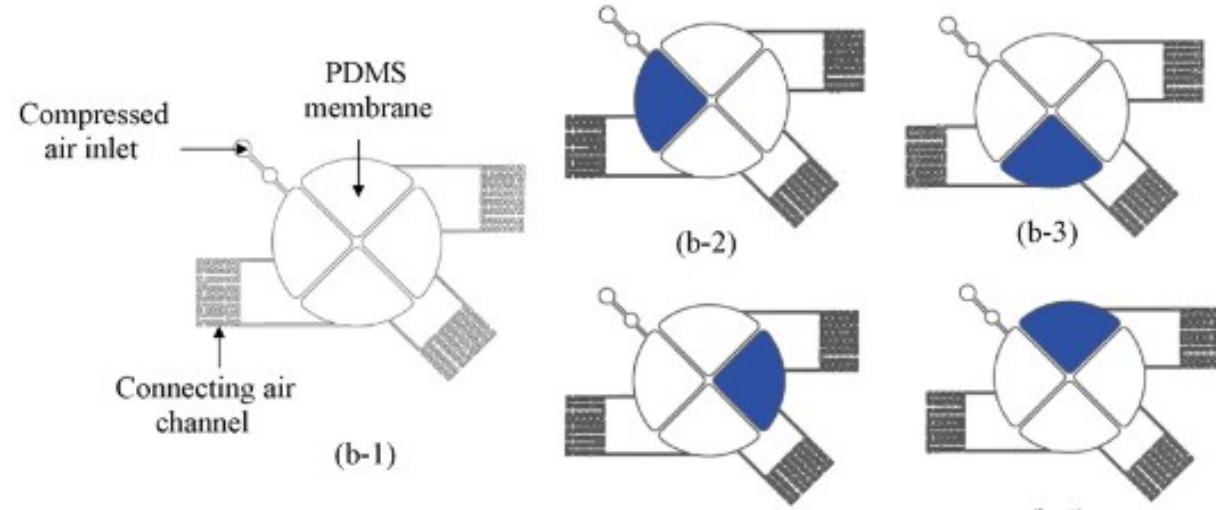

(b-4)

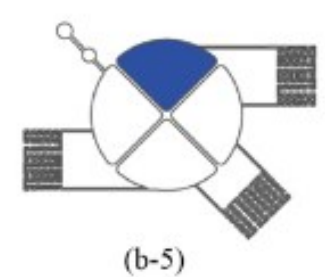

Figure 2-4 Magnetic bead-based microfluidic chip designed by Fang Lee et al. for the analysis of dengue associated antibodies in human plasma. (a) A photograph of the microfluidic chip, comprising one and two-way micropumps, a four-membrane type micromixer, and an on-chip microcoil array. The dimensions of this chip are measured to be $53 \mathrm{~mm} \times 37 \mathrm{~mm}$. (b-1) A schematic diagram of the four-membrane-type micromixer; (b-2)-(b-5) show the motion of the fluids in the micromixer [52]. 


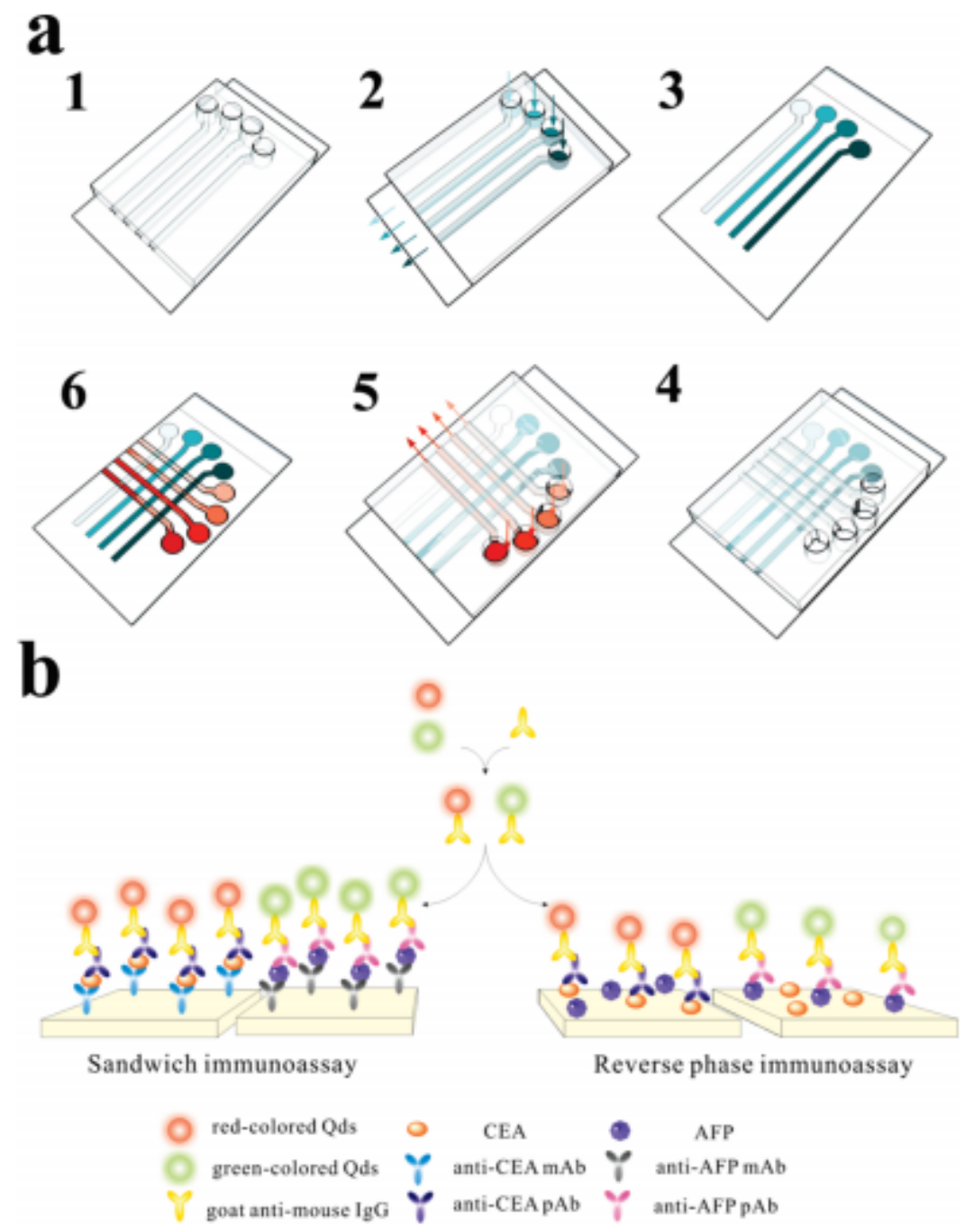

Figure 2-5 Microfluidic protein chip for an ultrasensitive and multiplexed assay of cancer biomarkers in human serum, in the format of both sandwich and reverse phase immunoassay. (a) Schematic illustration of the microchannel network. (1) First, a PDMS piece was placed in a silyated slide; (2) The solutions were flowed through the channels, and the first layer of protein was immobilized; (3) The first PDMS piece was removed; (4) The other PDMS piece was placed on the substrate with channels perpendicular to the first PDMS piece; (5) Other solutions were flowed; (6) A two dimensional array was formed above the chip. (b) Schematic illustration of a sandwich immunoassay and reverse phase immunoassay based on $\mathrm{CdTe} / \mathrm{CdS}$ quantum dots-IgG fluorescent probes and microfluidic protein chip [51].

\subsubsection{Point of Care Electrochemical Biosensors}

Electrochemical methods take advantage of the interaction of chemical species with electrodes or probes to obtain electrical signals, allowing for the quantification of target analytes 
present in the sample [39]. Electrochemical events can occur in two different orders, the first possibility is that the chemical reaction is triggered by passing an electrical current through the electrode system, the second is that the electrode responses are triggered by specific chemical reactions such as oxidation and reduction [39]. Applications of electrochemical microfluidic sensors include the diagnosis of tumor biomarkers (2-6) [33], and coagulation status monitoring [53].

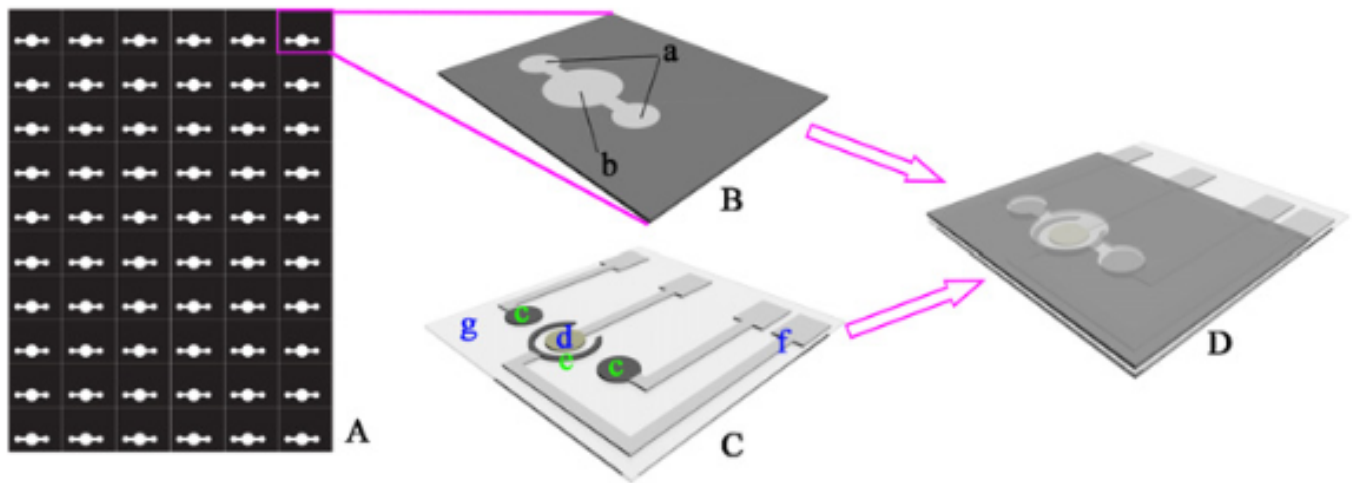

Figure 2-6 The schematic representation of microfluidic paper-based three-dimensional electrochemical device, developed by Wang et al. for the simultaneous diagnosis of two tumor markers: cancer antigen 125 and carcinoembryonic antigen. (A): Wax-patterned paper sheet; (B): the 3D-- $\mu$ PED, (a) paper working zones; (b) paper auxiliary zone. (C): Screen-printed electrodes, (c) carbon working electrodes; (d) $\mathrm{Ag} / \mathrm{AgCl}$ reference electrode; (e) carbon counter electrode; (f) silver conductive channel and pad; (g) transparent polyethylene terephthalate substrate. (D): After stacking, the paper working zones and the paper auxiliary zone will be aligned to the screen-printed working electrodes, counter and reference electrode, respectively [33].

\subsubsection{Point of Care Surface Acoustic Wave (SAW) Biosensors}

Surface acoustic wave biosensors rely on the modulation of surface acoustic waves to sense a physical phenomenon. In general, this devices generate and detect acoustic waves using interdigital transducers on the surface of a piezoelectric crystal [54]. SAW microfluidic immunosensors have been used in the detection of carcinoembryonic antigen (CEA) [55], [56] 

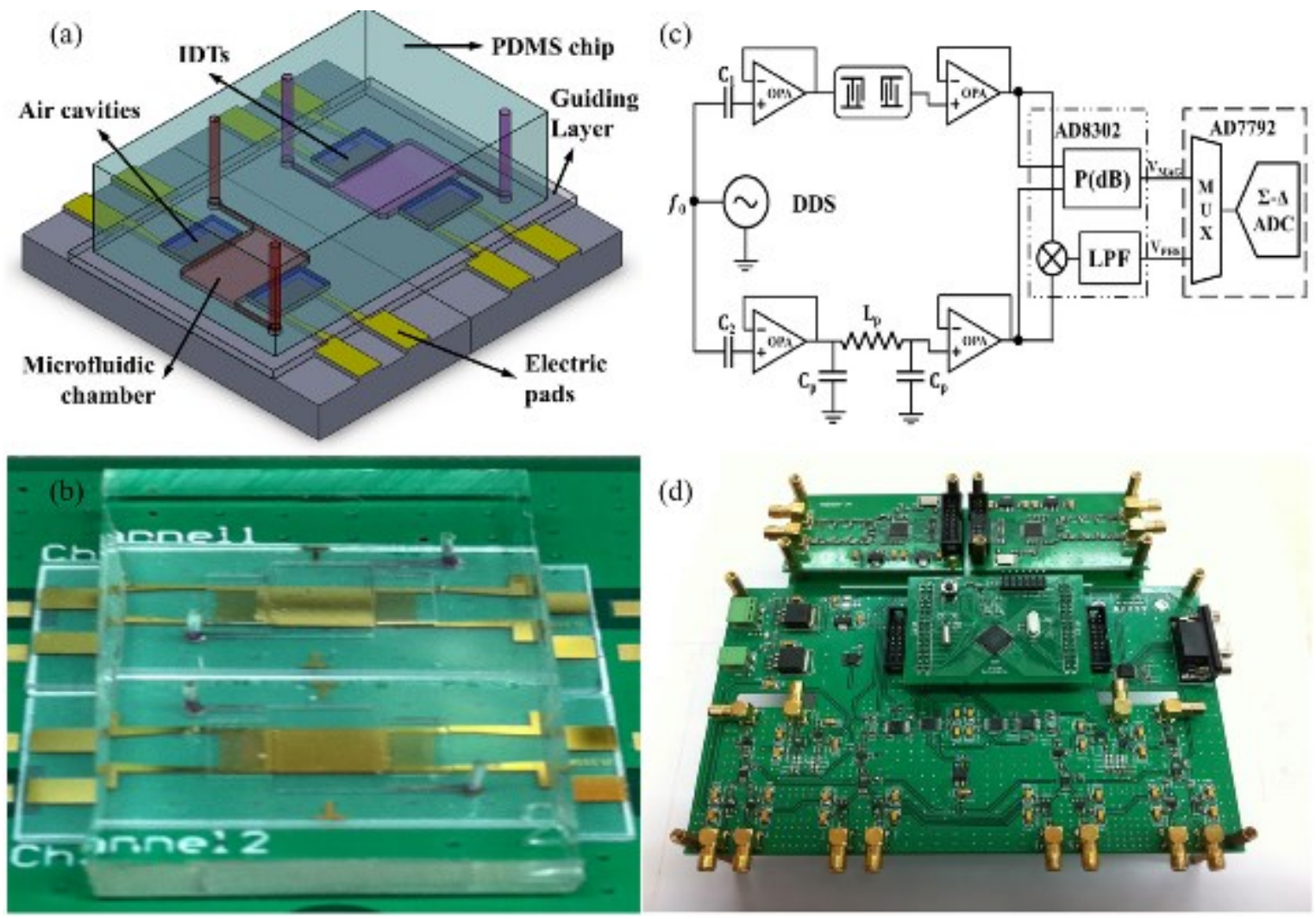

Figure 2-7 PDMS microfluidic chip coupled with Love Wave sensor. (b) Picture of the PDMS microfluidic chip. (c) Schematic of the circuit for Love Wave sensor measurement. (c) Printed circuit board [55].

\subsubsection{Point of Care Colorimetric Biosensors}

Colorimetric biosensors take advantage of the color change caused by chemical/biochemical reactions between the target analytes and the colorimetric probes [39], [57]. The advantage of this methodology for POC application lies in the ability of observing results with the naked eye, thus eliminating the need for bulky external detection systems [39], [58]. One of the most well-known colorimetric biosensors is the hCG pregnancy test strip [59], [60], however, these colorimetric biosensors have also been used for other applications such as multiplexing detection of Hepatitis B biomarkers (Figure 2-8) [61], human immunodeficiency virus (HIV) biomarkers (Figure 2-9) [62], the detection of papillomavirus [63] and anemia [64]. 


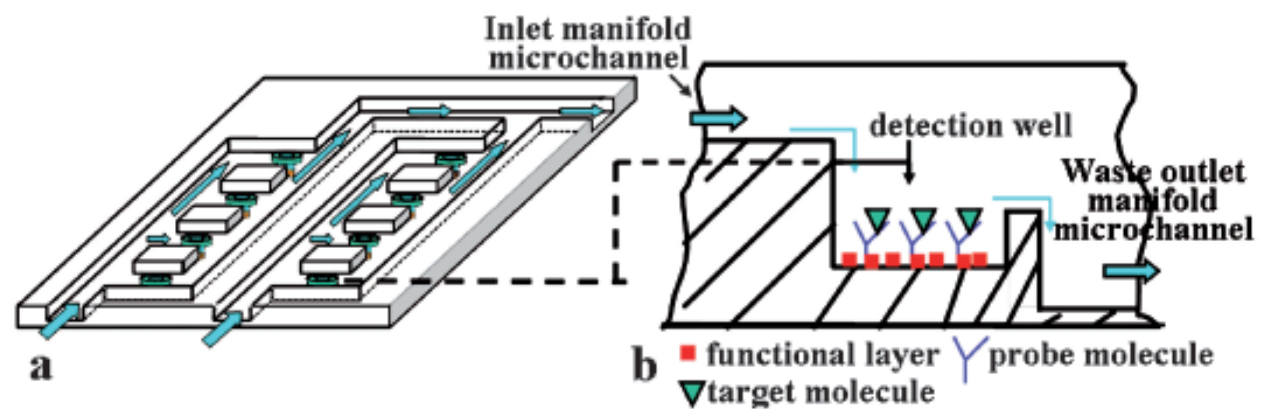

Figure 2-8 Modified PDMS microfluidic ELISA device for the multiplexing detection of IL-5, $\mathrm{HBsAg}$, and IgG in human serum. The microfluidic device encompassed several microchannels to guide the flow to a detection well, and then drain out to the waste outlet manifold by overflow, in such a way that backflow of the reacted fluid is eliminated, in order to avoid contamination. (a) Top view, (b) Section view of microfluidic ELISA device [61].
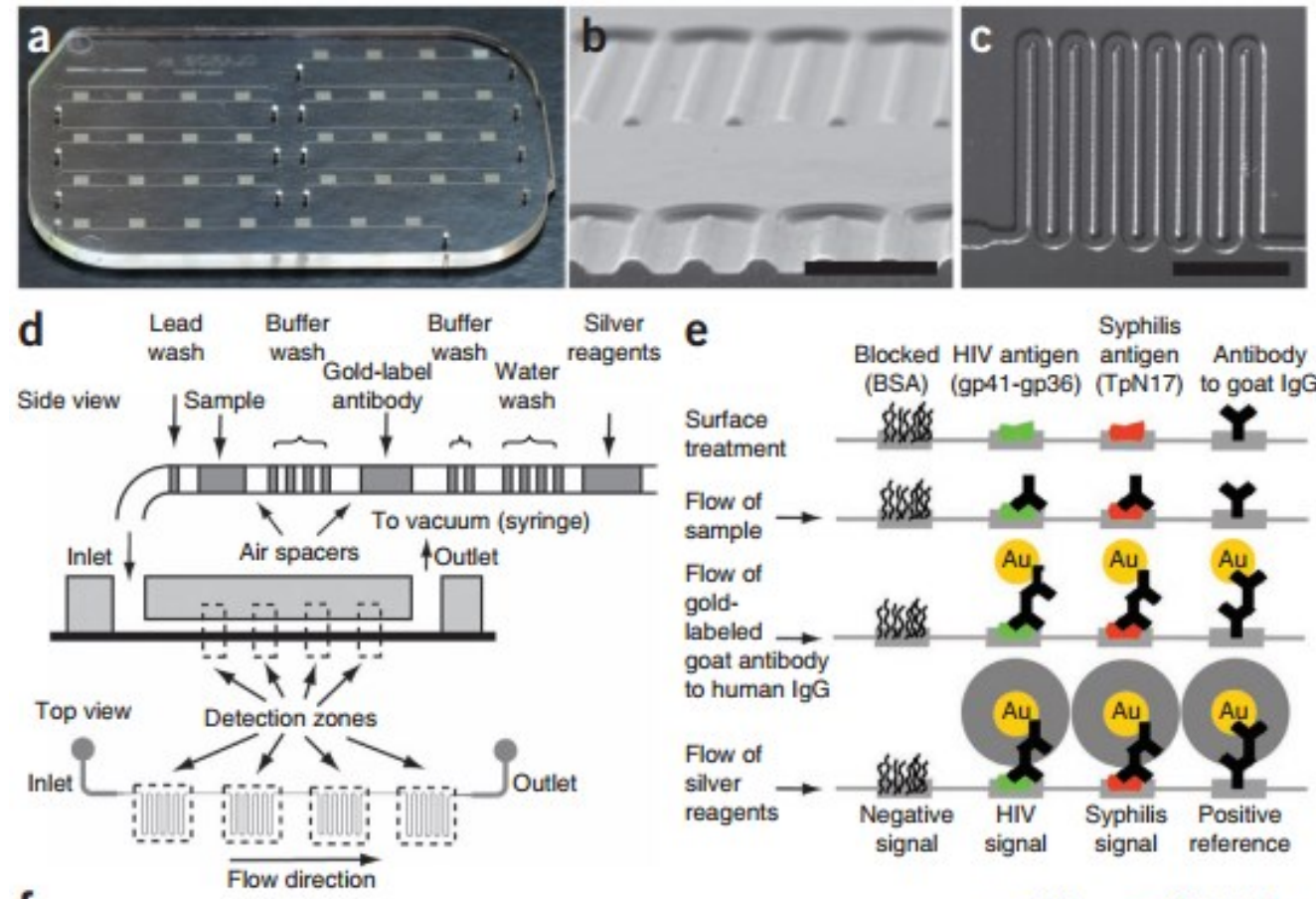

f
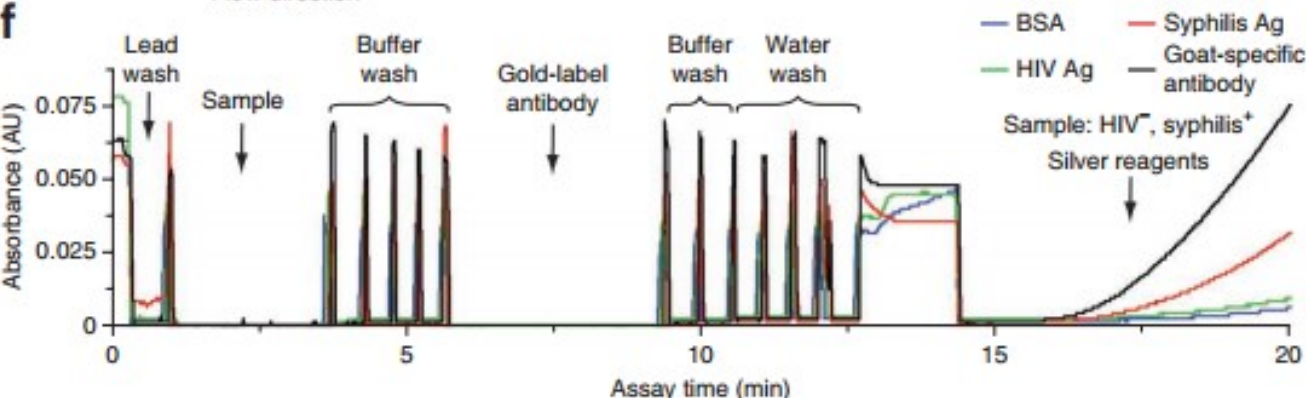

Figure 2-9 Schematic diagram and pictures of microfluidic device, and data on fluid handling of a POC ELISA-like assay for the simultaneous diagnosis of HIV and syphilis. (a) Picture of 
microfluidic chip. Each chip can accommodate seven samples (one per channel), with molded holes for coupling of reagent-loaded tubes. (b) Scanning electron microscope image of a crosssection of microchannels, made of injection-molded plastic. Scale bar, $500 \mu \mathrm{m}$. (c) Transmitted light micrograph of channel meanders. Scale bar, $1 \mathrm{~mm}$. (d) Schematic diagram of passive delivery of multiple reagents, which requires no moving parts on-chip. A preloaded sequence of reagents passes over a series of four detection zones, each characterized by dense meanders coated with capture proteins, before exiting the chip to a disposable syringe used to generate a vacuum for fluid actuation. (e) Illustration of biochemical reactions in detection zones at different immunoassay steps. The reduction of silver ions on gold nanoparticle-conjugated antibodies yields signals that can be read with low-cost optics (for quantification) or examined by eye. (f) Absorbance traces of a complete HIV-syphilis duplex test as reagent plugs pass through detection zones. High optical density (OD) is observed when air spacers pass through

the detection zones, owing to increased refraction of light compared to in the liquid filled channels. The train of reagents mimics the pipetting of reagents in and out of multiwall plates. This sample was evaluated (correctly against a reference standard) as HIV negative and syphilis positive. Ag, antigen [62].

\subsubsection{Point of Care Localized Surface Plasmon Resonance Biosensors}

Localized surface plasmon resonance (LSPR) optical phenomena is generated by the interaction of light resulting with conductive nanoparticles with a smaller size than the incident wavelength [65]. The electric field of incident light is used to excite electrons of a conduction band, resulting in coherent localized plasmon oscillations with a resonant frequency strongly dependant on the composition, size, geometry, dielectric environment and separation distance of the gold nanoparticles [65]. Localized Surface Plasmon Resonance Biosensors have been used in the diagnosis of prostate cancer [37], and microalbuminuria (Figure 2-10) [66]. 


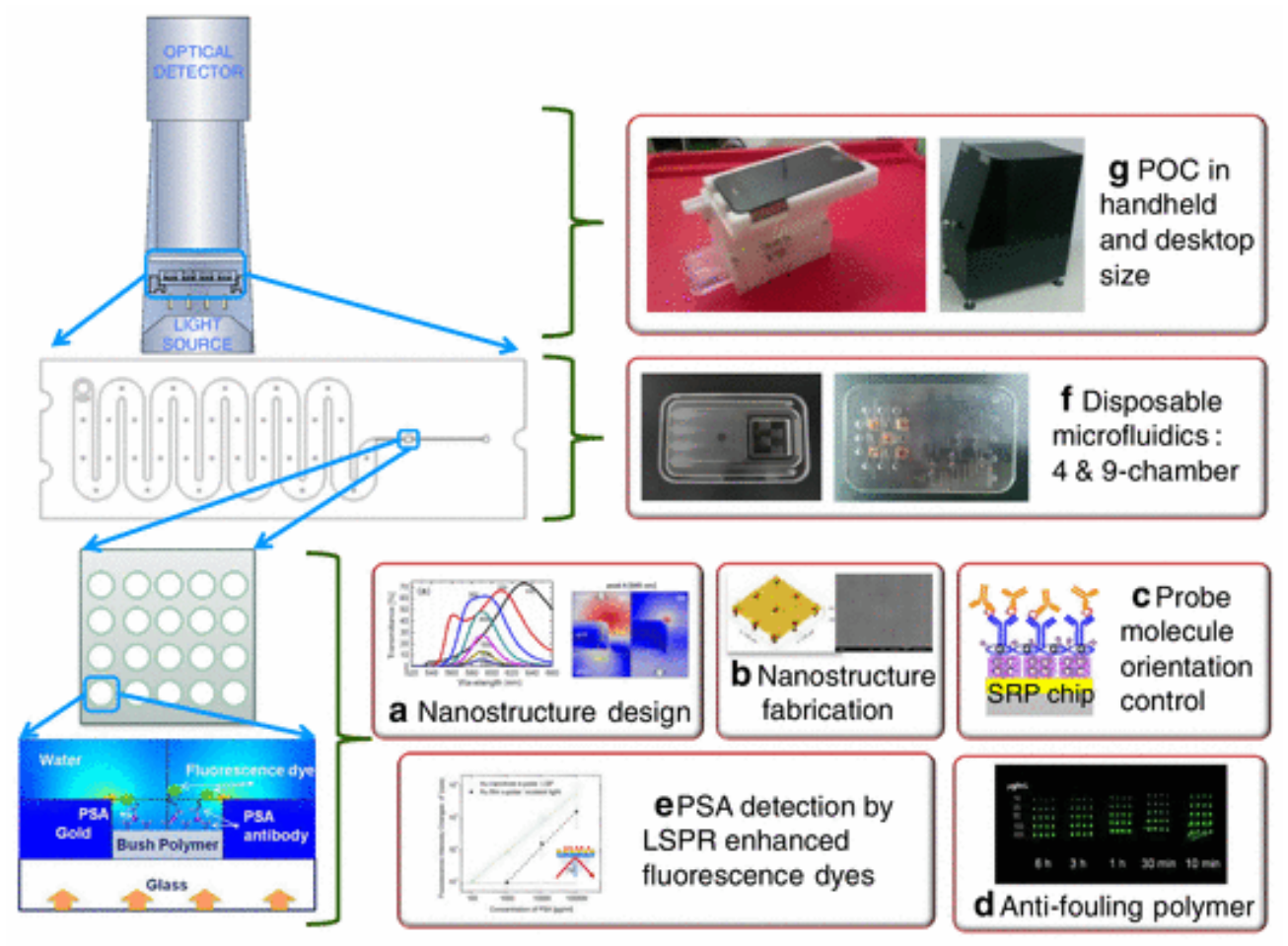

Figure 2-10 Nanobiosensor based on localized surface plasmon resonance (LSPR) for the detection of microalbuminuria. The principle (left) and technologies developed (right) for forming the LSPR POC system [37].

Table 2-1 further summarized state of the art of POC microfluidic platforms for biomarker detection [38]. 
Table 2-1 Summary of biomarker detection using microfluidic platforms [38].

\begin{tabular}{|c|c|c|c|c|c|c|}
\hline & Specific disease & Biomarkers & LOD & Detection method & $\begin{array}{l}\text { Microfluidic } \\
\text { platform }\end{array}$ & Ref. \\
\hline \multirow[t]{45}{*}{ Cancer } & \multirow[t]{11}{*}{ Colorectal carcinoma } & CEA & $250 \mathrm{fm}$ & Fluorescence & PDMS & 122 \\
\hline & & CEA & $3.5 \mathrm{ng} \mathrm{mL}^{-1}$ & Fluorescence & PDMS & 124 \\
\hline & & CEA & $0.01 \mathrm{ng} \mathrm{mL}^{-1}$ & Electrochemical & Paper & 31 \\
\hline & & CEA & $0.01 \mathrm{ng} \mathrm{mL}^{-1}$ & Electrochemical & Paper & 33 \\
\hline & & CEA & $0.05 \mathrm{ng} \mathrm{mL}^{-1}$ & Chemiluminescence & Paper & 32 \\
\hline & & CEA & $0.02 \mathrm{ng} \mathrm{mL}^{-1}$ & Chemiluminescence & Paper & 140 \\
\hline & & CEA & $0.5 \mathrm{ng} \mathrm{mL}^{-1}$ & ECL & Paper & 147 \\
\hline & & CEA & $0.8 \mathrm{pg} \mathrm{mL}^{-1}$ & ECL & Paper & 34 \\
\hline & & CEA & $1.25 \mathrm{ng} \mathrm{mL}^{-1}$ & SAW & PDMS & 48 \\
\hline & & CEA & $0.3 \mathrm{pg} \mathrm{mL}^{-1}$ & Electrochemical & Paper & 131 \\
\hline & & CEA & $1.7 \mathrm{pg} \mathrm{mL}^{-1}$ & Colorimetric & Paper & 111 \\
\hline & \multirow{9}{*}{$\begin{array}{l}\text { Hepatocellular } \\
\text { carcinoma }\end{array}$} & AFP & $250 \mathrm{fM}$ & Fluorescence & PDMS & 122 \\
\hline & & AFP & $0.01 \mathrm{ng} \mathrm{mL}^{-1}$ & Electrochemical & Paper & 31 \\
\hline & & AFP & $1 \mathrm{pg} \mathrm{mL}^{-1}$ & Electrochemical & PMMA & 133 \\
\hline & & AFP & $0.06 \mathrm{ng} \mathrm{mL}^{-1}$ & Chemiluminescence & Paper & 32 \\
\hline & & AFP & $1 \mathrm{ng} \mathrm{mL}^{-1}$ & Chemiluminescence & Paper & 140 \\
\hline & & AFP & $0.15 \mathrm{ng} \mathrm{mL}^{-1}$ & ECL & Paper & 147 \\
\hline & & AFP & $3.9 \mathrm{ng} \mathrm{mL}^{-1}$ & Fluorescence & PDMS & 124 \\
\hline & & AFP & $0.2 \mathrm{fg} / \mathrm{chip}$ & Fluorescence & PDMS & 123 \\
\hline & & AFP & $1.7 \mathrm{pg} \mathrm{mL}^{-1}$ & Colorimetric & Paper & 111 \\
\hline & \multirow[t]{5}{*}{ Ovarian cancer } & CA-125 & $0.05 \mathrm{ng} \mathrm{mL}^{-1}$ & Electrochemical & Paper & 31 \\
\hline & & CA-125 & $0.2 \mathrm{mU} \mathrm{mL}^{-1}$ & Electrochemical & Paper & 33 \\
\hline & & CA-125 & $0.33 \mathrm{ng} \mathrm{mL}^{-1}$ & Chemiluminescence & Paper & 32 \\
\hline & & $\mathrm{CA}-125$ & $0.6 \mathrm{U} \mathrm{mL}^{-1}$ & ECL & Paper & 147 \\
\hline & & CA-125 & $0.0074 \mathrm{U} \mathrm{mL}^{-1}$ & ECL & Paper & 35 \\
\hline & \multirow{9}{*}{ Prostate cancer } & PSA & $0.23 \mathrm{pg} \mathrm{mL}^{-1}$ & Electrochemical & PDMS & 49 \\
\hline & & PSA & $100 \mathrm{fg} \mathrm{mL}^{-1}$ & ECL & PMMA/PDMS & 36 \\
\hline & & PSA & $1 \mathrm{pg} \mathrm{mL}^{-1}$ & ECL & Paper & 34 \\
\hline & & PSA & $100 \mathrm{pg} \mathrm{mL}^{-1}$ & LSPR & POEGMA/glass & 37 \\
\hline & & PSA & $3.2 \mathrm{ng} \mathrm{mL}^{-1}$ & Colorimetric & PMMA & 108 \\
\hline & & PSA & $0.5 \mathrm{pM}$ & Fluorescence & PNIPAAm & 125 \\
\hline & & PSA & $100 \mathrm{pg} \mathrm{mL} \mathrm{m}^{-1}$ & ECL & POEGMA/glass & 37 \\
\hline & & II-6 & $0.30 \mathrm{pg} \mathrm{mL}^{-1}$ & Electrochemical & PDMS & 49 \\
\hline & & IL-6 & $10 \mathrm{fg} \mathrm{mL}^{-1}$ & ECL & PMMA/PDMS & 36 \\
\hline & \multirow[t]{3}{*}{ Pancreatic cancer } & CA-199 & $0.17 \mathrm{U} \mathrm{mL}^{-1}$ & ECL & Paper & 147 \\
\hline & & CA-199 & $0.0055 \mathrm{U} \mathrm{mL}^{-1}$ & ECL & Paper & 148 \\
\hline & & CA-199 & $0.06 \mathrm{U} \mathrm{mL}^{-1}$ & Chemiluminescence & Paper & 140 \\
\hline & \multirow[t]{2}{*}{ Breast cancer } & CA153 & $0.05 \mathrm{ng} \mathrm{mL}^{-1}$ & Electrochemical & Paper & 31 \\
\hline & & CA153 & $0.4 \mathrm{U} \mathrm{mL}^{-1}$ & Chemiluminescence & Paper & 140 \\
\hline & Human acute & HL-60 & 350 cells $\mathrm{mL}^{-1}$ & Electrochemical & Paper & 55 \\
\hline & leukemia & CCL-119 & 30 cells per $3 \mu \mathrm{L}$ & Chemiluminescence & PDMS & 142 \\
\hline & $\begin{array}{l}\text { Renal cancer } \\
\text { carcinoma }\end{array}$ & AQP1 & $24 \mathrm{pg} \mathrm{mL}^{-1}$ & LSPR & Paper & 154 \\
\hline & \multirow{3}{*}{ Bladder cancer } & APOA1 & $9.16 \mathrm{ng} \mathrm{mL}^{-1}$ & Fluorescence & PDMS & 120 \\
\hline & & TNF & $3.1 \mathrm{pg} \mathrm{mL}^{-1}$ & Fluorescence & Cyclo-olefin & 116 \\
\hline & & TNF & $\begin{array}{l}1000 \text { copies per } \\
4.7 \mathrm{~nL}\end{array}$ & Fluorescence & PDMS & 115 \\
\hline \multirow{11}{*}{$\begin{array}{l}\text { Infectious } \\
\text { disease }\end{array}$} & Hepatitis B & HBsAg & $100 \mathrm{pg} \mathrm{mL}^{-1}$ & Colorimetric & PDMS & 39 \\
\hline & Pseudorabies virus & Nucleic acid & $10 \mathrm{fg}_{\mu} \mathrm{L}^{-1}$ & Colorimetric & PDMS & 106 \\
\hline & Dengue virus & IgG/IgM & $21 \mathrm{pg} \mathrm{mL}^{-1}$ & Fluorescence & PDMS & 42 \\
\hline & Meningitis & ctrA gene & $\begin{array}{l}3 \text { copies per LAMP } \\
\text { zone }\end{array}$ & Fluorescence & PDMS/paper hybrid & 41 \\
\hline & Food-borne disease & SEB & $0.01 \mathrm{ng} \mathrm{mL}^{-1}$ & Chemiluminescence & Polycarbonate & 143 \\
\hline & Food-borne & S. enterica (aptamer) & $61 \mathrm{cfu} \mathrm{mL}^{-1}$ & Fluorescence & PDMS/paper hybrid & 95 \\
\hline & pathogens & S. aureus (aptamer) & $800 \mathrm{cfu} \mathrm{mL}^{-1}$ & Fluorescence & PDMS/paper hybrid & 95 \\
\hline & & E. coli & $\begin{array}{l}5 \times 10^{3} \text { cells per } \\
\mathrm{mL}\end{array}$ & Chemiluminescence & PMMA & 51 \\
\hline & & C. jejuni & $\begin{array}{l}1 \times 10^{5} \text { cells per } \\
\mathrm{mL}\end{array}$ & Chemiluminescence & PMMA & 51 \\
\hline & & IgG & $3.9 \mathrm{fM}$ & Electrochemical & Paper & 130 \\
\hline & & IgG & $10 \mathrm{pg} \mathrm{mL}^{-1}$ & Colorimetric & PDMS & 104 \\
\hline Other diseases & Diabetics & Glucose & $0.5 \mathrm{mM}$ & Colorimetric & Paper & 54 \\
\hline & & Glucose & $0.35 \mathrm{mM}$ & Electrochemical & Paper & 136 \\
\hline & Thyroid dysfunction & $\begin{array}{l}\text { Human thyroid stimulating } \\
\text { hormone }\end{array}$ & $68 \mathrm{pg} \mathrm{mL}^{-1}$ & Chemiluminescence & PDMS/glass & 141 \\
\hline & Cardiovascular & cTnI & $5 \mathrm{amol} / 30 \mu \mathrm{L}$ & Electrochemical & PDMS & 134 \\
\hline & disease & cTnI & $25 \mathrm{pg} \mathrm{mL}^{-1}$ & Electrochemical & $\begin{array}{l}\text { Vacrel@ } 8100 \\
\text { photoresist }\end{array}$ & 43 \\
\hline & & cTnI & $0.51 \mathrm{ng} \mathrm{mL}^{-1}$ & Colorimetric & PMMA & 105 \\
\hline & & cTnI & $24 \mathrm{pg} \mathrm{mL}^{-1}$ & Fluorescence & PMMA & 50 \\
\hline & & CRP & $\begin{array}{l}307 \text { amol per } \\
30 \mu \mathrm{L}\end{array}$ & Electrochemical & PDMS & 134 \\
\hline & & CRP & $0.30 \mathrm{ng} \mathrm{mL}^{-1}$ & Colorimetric & PMMA & 105 \\
\hline & & NT-proBNP & $0.03 \mathrm{ng} \mathrm{mL}^{-1}$ & Electrochemical & & 135 \\
\hline & & NT-proBNP & $0.24 \mathrm{ng} \mathrm{mL}^{-1}$ & Colorimetric & PMMA & 105 \\
\hline & Alzheimer's disease & ApoE & $12.5 \mathrm{ng} \mathrm{mL}^{-1}$ & Electrochemical & PDMS & 45 \\
\hline & Bronchial asthma & IL-5 & $100 \mathrm{pg} \mathrm{mL}^{-1}$ & Colorimetric & PDMS & 39 \\
\hline & Neuromyelitis optica & $\operatorname{IgG}$ & $100 \mathrm{pg} \mathrm{mL}^{-1}$ & Colorimetric & PDMS & 39 \\
\hline & & Uric acid & $0.52 \mathrm{mM}$ & Electrochemical & Paper & 136 \\
\hline & & Uric acid & $8.1 \mathrm{ppm}$ & Colorimetric & Paper & 112 \\
\hline & & Lactate & $1.76 \mathrm{mM}$ & Electrochemical & Paper & 136 \\
\hline
\end{tabular}




\subsection{Limitations}

While the development of microfluidic technology for diagnostics and disease monitoring has evolved rapidly over the past years, to this date, these technologies still face several challenges for becoming truly viable for POCT [27]. For one, many of the technologies available still require some degree of training for handling the device, sample treatment, and even expensive external equipment for flow initiation and control as well as detection, and do not meet the portability requirement, which are all great limitations especially for low-resource settings. Detection methods like colorimetry are simple and low cost, however, they might not be as sensitive and somewhat compromise quantitation [39]. Other significant challenges remain in working with human sample matrices [27]. Also, each different human sample type and each disease biomarker require special assay development [27]; it is very complex to recreate a versatile platform which can be used with a broad spectrum of input species or clinical patient samples [27].

\subsection{Chapter Summary}

In summary, a number of microfluidic platforms have been developed for the rapid detection of disease biomarkers, using materials ranging from paper to polymers and hybrid materials [39]. Some of these platforms are designed to perform individual steps, and can only be used to test human serum and not whole blood, but the critical challenge is to integrate these steps into a sensitive and selective integrated device that meets all the POC requirements including portability, low cost, and ease of handling [27]. To date only a small number of devices that offer total analysis on chip, including reagent storage, and sample collection and preparation, or the ability to test crude real-world samples (e.g. blood, urine, and saliva) [39], 
[67]-[69]. The microfluidics community is in need of integrated POC solutions that can be used not only in research laboratory settings, but can also be delivered directly to patients. These integrated POC solutions would help bridge the healthcare gap between developed and developing countries [70]. 


\section{Chapter 3 Capillary Systems Theory}

\subsection{Capillary Phenomena}

Capillary phenomena designate the forces produced by interfacial tensions $(\mathrm{N} / \mathrm{m})$ at the interface of at least two immiscible phases, and the spontaneous transformation and dissipation of the interfacial energy into motion of a liquid [4], [71], [72]. These effects become predominant at small scales, where the forces exerted by the molecules at the interface surpass the forces arising from the molecules in the bulk [4]. Even though significant only at micrometer scales, capillary phenomena forms part of our everyday life [4].

Capillarity has been studied since da Vinci's time [71]. Leonardo da Vinci's observation of the capillary rise of liquid in tubes was the first recorded observation of capillary action [71]. In his book of 1759, Giovani Batista Clemente Nelli describes that a former student of Galileo, Niccolo Aggiunti (1600-1635), had also studied capillary action in mosquitoes, butterflies, and bees feeding [73]. Other studies soon followed [74]-[79]. Mathematicians Honoré Fabri and Jacob Bernoulli thought that liquids rose in capillaries because air couldn't enter capillaries as easily as liquids, so the air pressure was lower inside capillaries [80], [81]. Other scholars like Isaac Vossius, Giovanni Alfonso Borelli, Louis Carré, Francis Hauksbee, and Josia Weitbrecht thought that the particles of liquid were attracted to each other and to the walls of the capillary [82]-[89]. It took until 1805, for two investigators, Thomas Young and Pierre-Simon Laplace, to successfully quantify capillary action [90], [91]; they derived the Young-Laplace Equation of capillary action. Carl Friedrich Gauss then determined the boundary conditions governing capillary action in 1830 [92]. The effect of the meniscus on a liquid's vapor pressure, and the interaction between two immiscible liquids were determined by William Thomson and Franz Ernst Neumann respectively [93], [94]. 


\subsubsection{Wetting and Interfacial Free Energy}

When a small drop of liquid is put onto a flat solid surface, two distinct outcomes, representative of two different equilibrium regimes can occur [95], [96]. The first is partial wetting, where the liquid drop has a finite contact angle $\alpha$ with the surface; the second is total wetting, where the liquid spreads out as a thin molecular film [4]. The magnitudes of the free energy per unit area $\left(\mathrm{J} / \mathrm{m}^{2}\right)$ of each interface can be related via the contact angle $\alpha$ of the liquid to the surface, using Young's equation [4].

$$
\gamma_{L V} * \cos (\alpha)=\gamma_{S V-} \gamma_{S L}(\mathbf{1})
$$

Where $\gamma_{L V}$, SV, SL are free energy per unit area of the liquid-gas, solid-gas, and solid-liquid interfaces respectively (Figure 3-1) [4].

(a)

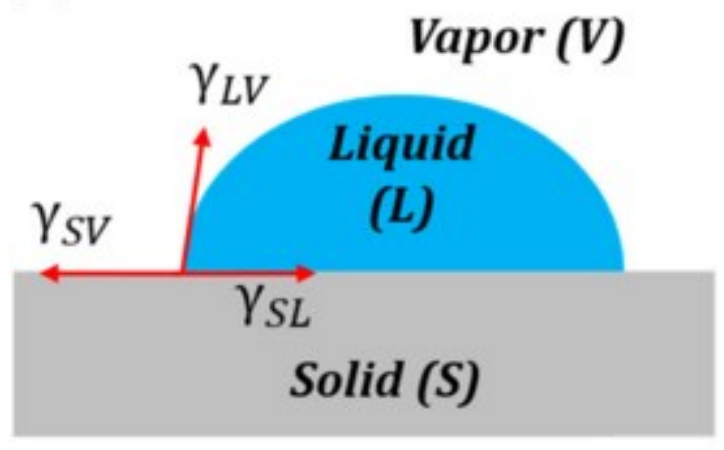

Figure 3-1 (a) Surface tension forces at the three-phase line on a non-deformable solid surface [97].

If the boundary between wettable and non-wettable is $90^{\circ}$ [4]; a surface is wettable when the magnitude of the contact angle is lower than $90^{\circ}$, and non-wettable if the contact angle is greater than $90^{\circ}$ [4]. For the case of water, the wettability can be defined as hydrophilicity $\left(\alpha<90^{\circ}\right)$ and hydrophobicity $\left(\alpha>90^{\circ}\right)[4]$. 


\subsubsection{Dynamics of capillary flow in a microchannel}

In autonomous capillary systems (CSs) liquids are displaced by means of capillarity [98]. Their proper functioning requires displacing accurate volumes of liquids with precise flow rates [98]. The flow velocity of a liquid in a conduit can be derived from solving the Navier-Stokes equations [4].

The flow rate of a liquid is a function of the viscosity of the fluid, the capillary pressure, and the total flow resistance of the flow path (governed by the device's geometry), and can be calculated by integrating the flow velocity (Equation 2) [4], [99].

$$
Q=\frac{\Delta P}{8 \eta} * \frac{A * R_{H}}{L}(\mathbf{2})
$$

where $\eta$ is the viscosity of the fluid, $\mathrm{R}_{\mathrm{F}}$ is the total resistance of the flow path, and $\Delta \mathrm{P}$ is the differential pressure between inlet and outlet, $\mathrm{L}$ is the length, $\mathrm{A}$ the area, and $R_{H}$ is the hydraulic radius of the microchannel (Equation 5). During filling by capillary action $\Delta \mathrm{P}$ can be replaced with the pressure generated at the liquid meniscus, or capillary pressure $\left(\mathrm{P}_{c}\right)$ [4], [98].

The geometric and constant parameters can be regrouped for convenience into a unique parameter that we call the total flow-rate resistance, resulting in a simplified equation for flow rate (Equation 3) [4].

$$
Q=\frac{1}{\eta} * \frac{\Delta P}{R_{F}}=\frac{1}{\eta} * \frac{P_{C}}{R_{F}}(\mathbf{3})
$$

The total flow rate resistance $\left(\mathrm{R}_{\mathrm{F}}\right)$ of the flow path for a rectangular microchannel is not trivial to calculate, but it can be approximated by a linear term as long as $a<b$ (Equation 4) [4], [100]. 


$$
\begin{gathered}
R_{F}=\left[\frac{1}{12}\left(1+\frac{5 a}{6 b}\right) * \frac{a b R_{H}^{2}}{L}\right]^{-1} \\
R_{H}=\frac{a b}{a+b}(5)
\end{gathered}
$$

Where $\mathrm{a}$ and $\mathrm{b}$ are the depth and width of microchannel respectively [4], [98]. When filling the microchannel due to capillary action, the parameter L corresponds to the filled length of the channel, consequently, it increases with the advancement of the filling front of the liquid in the channel [4]. For a system with varying cross-sections connected in series, the total $\mathrm{R}_{\mathrm{F}}$ of the system can be calculated as the sum of the different components with constant cross-sections [4].

\subsubsection{Capillary Pressure}

The capillary pressure $\left(\mathrm{P}_{\mathrm{c}}\right)$ is the difference in pressure across the interface between two immiscible fluids, in this case plasma and air, and can be described by the Young-Laplace equation [4]. For a microchannel with a rectangular cross section, the curvature along the depth

and width axes contributes to the meniscus pressure [4]. $\mathrm{P}_{\mathrm{c}}$ can then be calculated using Equation 6 [4], [101].

$$
P_{c}=-\gamma\left(\frac{\cos \alpha_{b}+\cos \alpha_{t}}{a}+\frac{\cos \alpha_{l}+\cos \alpha_{r}}{b}\right.
$$

where $\gamma$ is the surface tension of liquid, and $\alpha_{b, t, 1, r}$ are the contact angles of the liquid on the bottom, top, left, and right wall of the microchannel respectively. The dynamic contact angles of the liquid with the walls of the microchannel depend on the speed of flow, as well as the receding or advancing state of the interface [4]. 
When using the static contact angles of the liquid with the substrate to calculate $\mathrm{P}_{\mathrm{c}}$, the four contact angles are assumed to be equal in magnitude, simplifying the calculation (Equations 7-9).

$$
\begin{array}{r}
P_{c}=\gamma\left(\frac{1}{R_{\text {Height }}}+\frac{1}{R_{\text {Width }}}\right)(7 \\
R_{\text {Height }}=\frac{a}{2 * \cos \alpha}(\mathbf{8}) \\
R_{\text {Width }}=\frac{b}{2 * \cos \alpha}(\mathbf{9})
\end{array}
$$

Where $\mathrm{R}_{\text {Height }}$ and $\mathrm{R}_{\text {Width }}$ are the minor and mayor radii of curvature respectively, and $\alpha$ is the static contact angle.

\subsubsection{Viscosity}

Viscosity can be thought of as a measure of fluid friction: the greater the viscosity, the greater its resistance to flow [102]. More formally, it is a measure of its resistance to deformation by shear or tensile stress arising from the collision between neighboring particles in a fluid, moving at different velocities [50]-[52]. For Newtonian fluids (e.g blood plasma), its magnitude can be calculated as the ratio of the shearing stress to the velocity gradient in a fluid [4], [102][104]. For the case of Non-Newtonian fluids (e.g blood), the relationship between shear stress and the velocity gradient is no longer linear, and therefore requires more complex calculations [102].

Viscosity is mainly a function of the material, but it also varies with temperature: increased temperature will result in less viscous fluids [102], [103]; this occurs because as temperature increases, the average speed of the molecules in the liquid increases as well, 
resulting in a reduction of intermolecular forces [103]. However, in this thesis the temperature is assumed to be constant during the operation of microfluidic devices [102].

The behavior of whole blood is that of a non-Newtonian fluid, meaning that its viscosity is dependent on shear rate (shear thinning or thickening) [105]. Shear rate is a function of the blood flow velocity, and as a result, when blood moves quickly (e.g. peak-systole), the shear is high and the blood is thinner, and when it moves slowly (e.g. end-diastole), the shear is lower, and blood becomes thicker due to red blood cell aggregation [105]. In conclusion, the viscosity of blood dynamically changes during each cardiac cycle [105].

On the other hand, serum and plasma are Newtonian fluids, so their viscosity does not change with the flow velocity, and therefore it is easier to measure [105], [106]. The viscosity of plasma is determined by its water-content and macromolecular components [106]. Since plasma is a highly concentrated protein solution, the proteins will have an effect in its viscosity based on their molecular weight and structure [106]; higher molecular weights will result in higher aggregating capacity, and together with their sensitivity to higher temperatures or $\mathrm{pH}$, will result in higher plasma viscosity values [106]. Normal plasma viscosity ranges between 1.10 and 1.30 $\mathrm{mPa} \cdot \mathrm{s}$ at $37^{\circ} \mathrm{C}$, however, alterations might occur due to pathologies that cause inflammations (e.g. rheumatoid arthritis), tissue injuries, unstable angina pectoris and stroke, and sometimes their higher values are associated with the progression of the disease and higher rate of major adverse clinical events [106].

For the purpose of our study, the viscosity of both Phosphate-buffered saline (PBS) and plasma was measured with the aid of a viscometer at room temperature, and the values were found to be 1.04 and $1.66 \mathrm{mPa} \cdot \mathrm{s}$ respectively. The measured value for the viscosity of PBS is comparable to reported by Fluxion Biosciences [107]. However, the measured value for the 
viscosity of human blood plasma is slightly higher than normal levels, which can be consequence of pathologies, inflammations, amongst others [106].

\subsubsection{Hagen-Poiseuille's Law}

In fluid dynamics, the Bernouilli's equation is an application of the law of conservation of mechanical energy to a moving fluid [108]. This is the simplest model for the flow of a fluid through a channel [108]; however, it assumes that the fluid is incompressible and that there is no friction effect on it as it moves in the channel, which is not the case in microfluidics [108].

The Hagen-Poiseuille equation describes a physical law that relates the pressure drop in an incompressible, Newtonian fluid flowing in the laminar regime through a channel with constant cross-section (no acceleration) [102]. All which are characteristic of microfluidic systems [102].

Ideally, Hagen-Poiseuille's law applies for perfectly straight and infinitely long channels [102]; however, it can be used as a reasonable estimate for channels with finite length (L) as long as $\mathrm{L}$ is the prevalent dimension of the geometry [102].

\subsubsection{Analogy Between Fluidic and Electric Circuit}

The behavior of fluids in channel networks has a great resemblance with currents in electric circuits [109]. In electronics, the resistance $\left(\mathrm{R}_{\mathrm{E}}\right)$ of linear resistors can be described by Equation 10:

$$
R_{E}=\frac{\rho_{E^{*} L_{E}}}{A}(10)
$$

Where $\rho_{\mathrm{E}}$ is the resistivity of the conductor in ohm, $\mathrm{L}_{\mathrm{E}}$ is the length of the conductor in meters, and A the cross-sectional area in meters squared [102]. 
The voltage (V) across the conducting material can be calculated using Ohm's law, which says that $\mathrm{V}$ is equal to the current (I) flowing through the conductor times $\mathrm{R}_{\mathbf{E}}$. Ohm's law finds its fluidic counterpart in Hagen-Poiseuille's law, which is described by Equation 11 as follows:

$$
\Delta p=R_{h} * Q(\mathbf{1 1})
$$

Where $\Delta \mathrm{p}$ is the pressure drop across the microchannel in $\mathrm{Pa}, \mathrm{Q}$ the volumetric flow rate in $\mathrm{m}^{3} / \mathrm{s}$, and $\mathrm{R}_{\mathrm{h}}$ the hydraulic resistance in $\mathrm{Pa}^{*} \mathrm{~s} / \mathrm{m}^{3}$ [102]. The hydraulic resistance of a rectangular microchannel can be approximated by:

$$
R_{h}=\frac{\Pi_{G} * \eta * L_{C}}{b * a^{3}}(\mathbf{1 2})
$$

Where $L_{C}$ is the length of the channel in meter, $a$ and $b$ are the depth and width of microchannel respectively in meter, and $\Pi_{\mathrm{G}}$ is a dimensionless parameter defined as:

$$
\Pi_{G}=12 *\left(1-\frac{192 * a}{\pi^{5} * b} * \tanh \left(\frac{\pi * b}{2 * a}\right)\right)^{-1}(13)
$$

In a similar way to electric resistors, for $\mathrm{N}$ fluidic resistors (microfluidic channels) arranged in series, an equivalent single fluidic resistor has a hydraulic resistance equal to the sum of the $\mathrm{N}$ hydraulic resistances, because each resistor is carrying the same volumetric flow from one end to the other [102]. For parallel connected fluidic resistors the equivalent single fluidic resistor has a hydraulic resistance equal to the reciprocal of the sum of reciprocals of each hydraulic resistance [102].

German physicist Gustav Kirchhoff greatly contributed to the understanding of electric circuits by stating two fundamental laws [110]. The first law is commonly called Kirchhoff's current law (KCL), and it says that at any point of the electric circuit, the sum of the currents flowing towards a node is equivalent to the sum of currents flowing out of the node [110]. This 
law has a counterpart in fluidic circuits: the conservation of mass, which states that the sum of the flows into a node should be equal to the sum of the flows leaving the node [102].

In a similar way, the second law (Kirchhoff's voltage law) also has a counterpart in fluidic networks, which implies that the sum of each pressure drop along the closed path is zero [102]. 


\section{Chapter 4 Capillary Driven Microfluidic Platform}

\subsection{Background}

Microfluidic systems have the potential of significantly changing how modern biology is done, due to their unique ability of integrating entire laboratory procedures onto a single chip [111]. These systems comprise a series of different basic components in order to perform the required functionality and the basic microfluidic operations [112], [113]. These basic components include: a method of introducing reagents and samples, methods for fluid transport, and a method for combination and mixing [113]. Some systems further extend their capabilities by integrating other components such as detection and in-chip sample processing [113].

Miniaturization leads onto the area of small Reynolds numbers, where fluid dynamics are very different from the macro scale, and different forces and effects dominate [111], [114]; the effects that become dominant in the micro scale fluidics include laminar flow, diffusion, fluidic resistance, surface area to volume ratio, and surface tension [111], [114]. Microfluidic systems should be designed in such a way that they take advantage of forces and effects that work on the micro scale [111].

Here, we describe a strategy to initiate fluid flow by taking advantage of surface tension, control the rate of flow by modifying both the hydraulic resistance encoded by the flow resistor and modifying the PDMS surface properties by functionalizing it using Pluronic F127, separate plasma from human whole blood, and potentially perform biomarker detection. 


\subsection{Design of an Integrated Lateral Flow Device}

A lateral flow device (LFD) operated under the capillary force was designed for the detection of circulating biomarkers. The integrated LFD is composed of two layers, a bottom fluid layer and a top interfacing layer (Figure 4-1). The fluidic layer consists of a membrane-based plasmaseparation unit, a capillary pump (CP) for plasma extraction/delivery unit, a detection chamber, a flow resistor, and a second capillary pump for assisting fluid flow and waste collection. The interfacing layer consists of the reservoir for whole blood loading, an embedded prefunctionalized glass substrate, and waste collection. The plasma separation membrane will be trimmed and put in a close contact with the microstructures in the $1^{\text {st }}$ capillary pump.

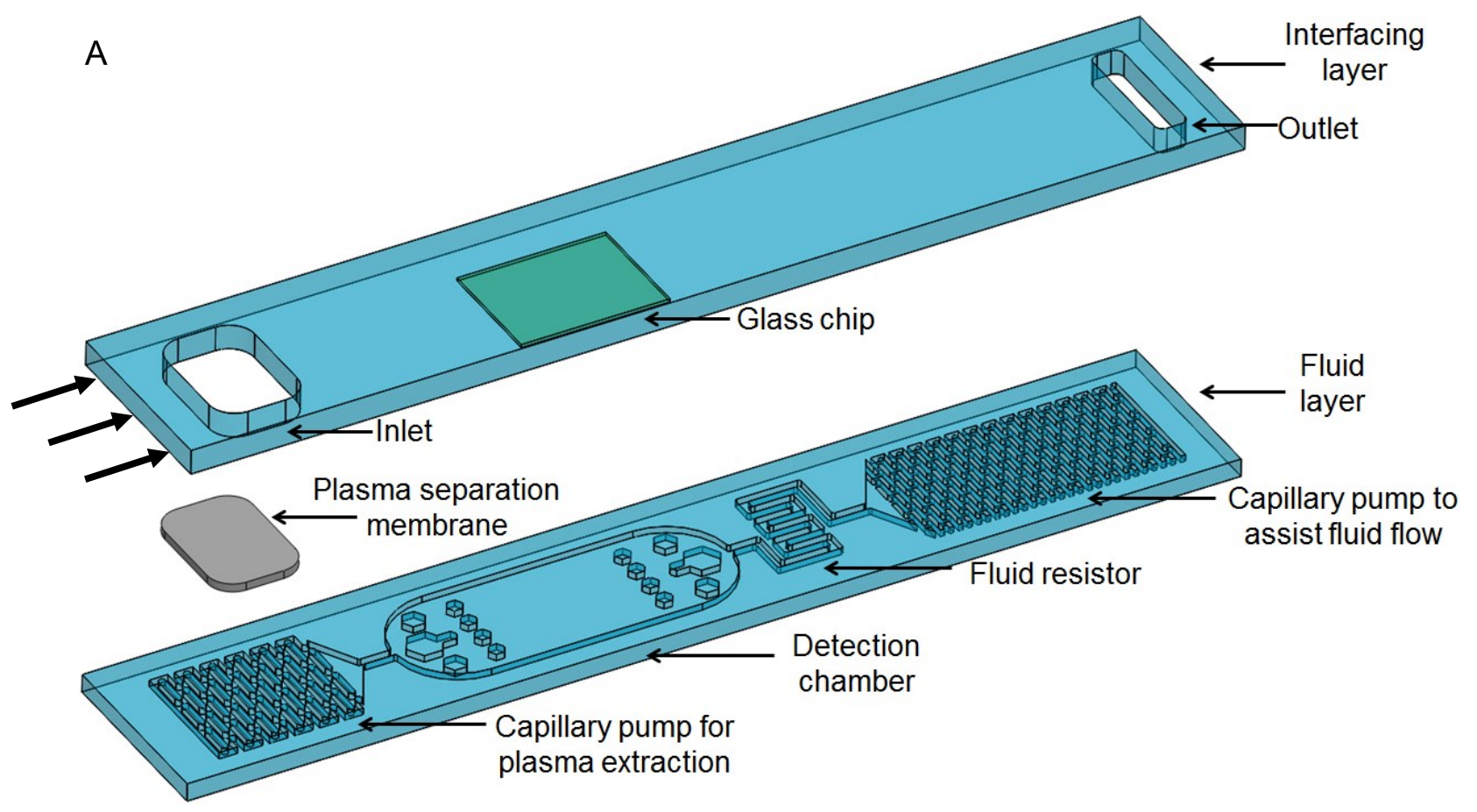




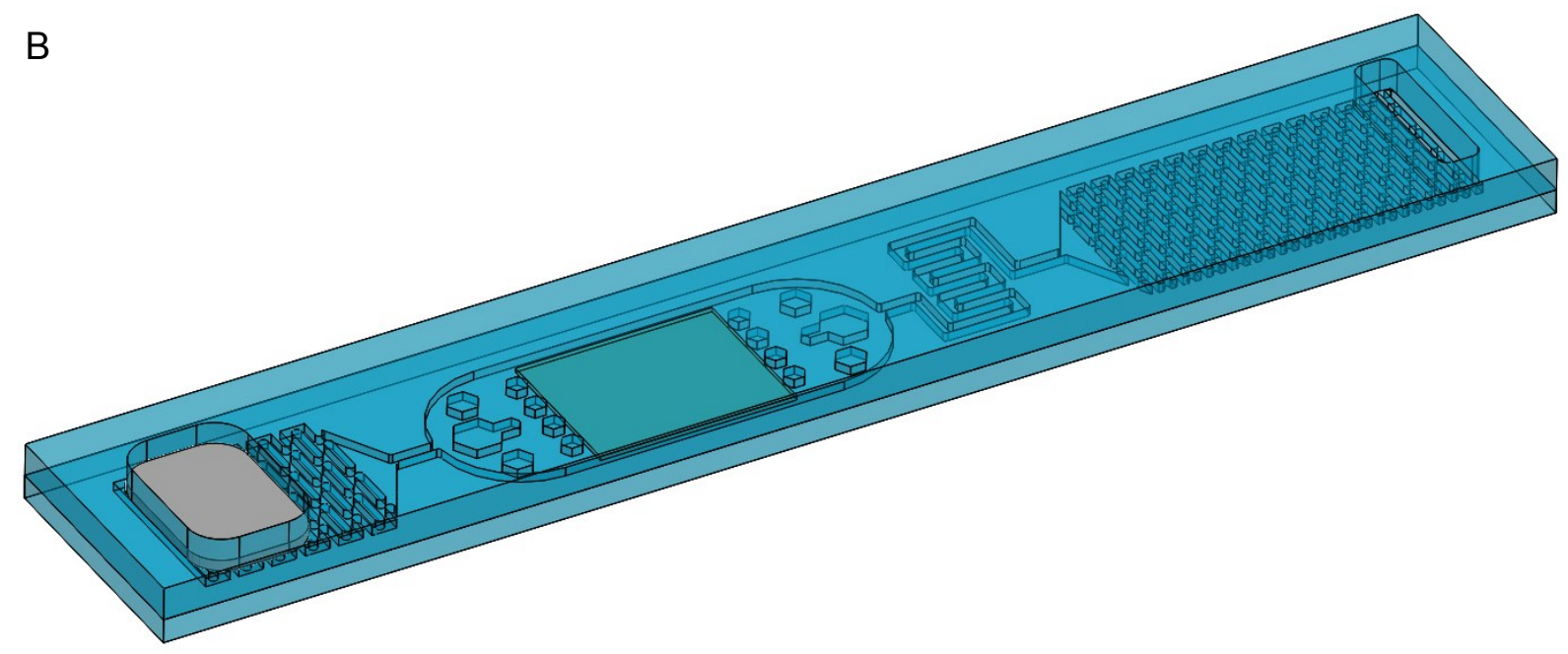

Figure 4-1 A) Schematic diagram of lateral flow device (LFD) for detection of single biomarker in whole blood sample. The first capillary pump (CP1) is partially covered by a GR plasma separation membrane, and it is designed for plasma extraction, while the second capillary pump (CP2) is designed to assist the fluid flow. Surface-immunoassays can be performed directly on the sealed chip by patterning capture antibodies on the gold chip placed on top of the detection chamber area. B) Schematic of assembled LFD. The black arrows indicate the flow direction.

Ideally, the capillary pumps should generate smooth flow of the fluid in the capillary system (CS), filling the CS completely without entrapping air. Therefore the $\mathrm{CP}$ must have low flow resistance and high capillary pressure [98]. Advanced capillary pumping structures can further enhance the pumping capabilities of the CS [98]. The pioneer work for capillary pumping systems had been demontrated by Delamarche et al., in which the characteristic dimensions of the structures, width and spacing, affect the progression rates of the liquid in the vertical and horizontal directions [9], [98]. In our LFD, the $1^{\text {st }} \mathrm{CP}$ for plasma extraction was designed with symmetric elongated structures $(260 \mu \mathrm{m}$ wide, $1041 \mu \mathrm{m}$ long, and with a $260 \mu \mathrm{m}$ spacing between structures) to control the filling front of the fluid, and $2{ }^{\text {nd }} \mathrm{CP}$ for assisting fluid flow and waste collection was designed with symmetric elongated microstructures as well $(160 \mu \mathrm{m}$ wide, $640 \mu \mathrm{m}$ long, and with a $160 \mu \mathrm{m}$ spacing between structures). In capillary pumps with symmetric structures, the resulting filling front is parallel to the microstructures [98]. For an enclosed 
capillary pump, such as ours, there is a risk of entrapping air; symmetric structures reduce this risk since the filling fronts of liquid converge from the edges of the $\mathrm{CP}$ towards its center [98].

Accurate control of the flow is a key in providing enough time for protein binding to antibodies and biomarker detection; fluidic resistances of microchannels allow modulating the flow rate passively [115]. In our case, the resistance of fluid resistor was modified by means of varying the length of the flow resistor. Lengths studied ranged from $0.324 \mathrm{~cm}$ to $37 \mathrm{~cm}$, while the width and depth of the channel were kept constant at $200 \mu \mathrm{m}$ and approximately $20 \mu \mathrm{m}$ respectively.

\subsection{Chapter Summary}

The novelty of this work lies in the high degree of flexibility and the integration of all functions required to perform a sandwich immunoassay through POC techniques. The LFD can separate human plasma from a droplet of whole blood, initiate and control the flow using a combination of resistance and surface modification techniques, and has the potential for detection of different biomarkers using a wide range of methodologies (e.g., colorimetric methods, fluorescence), all in a single chip. The LFD is portable, easy to handle, and low cost since it does not rely on expensive external equipment. 


\section{Chapter 5 Fabrication and Testing}

\subsection{Fabrication}

\subsubsection{Background}

Materials dictate the properties of the microfluidic flow path in a large extent [3]; they influence flow rate, capillary pressure, wetting, optical properties, adhesion of biomolecules, cost, and the fabrication methods required [3]. Glass and silicon were the first generation microfluidics materials thanks to their capillary electrophoresis and solvent-involved applications; the downside to these materials is that they are expensive for microfabrication, and for the case of silicon, its opacity to visible and ultraviolet light, which present a limitation for conventional optical methods of detection [16]. Since then, a wide of materials and different fabrication techniques have been explored (Figure 5-1) [3], [116]. Elastomeric Polymer materials are widely used in microfluidics due to their ease of fabrication and surface modification, optical transparency to visible and UV light, biocompatibility, flexibility, low cost, and it can be used to pattern both planar and non-planar surfaces [147], [149]; they also allow for complicated and parallel fluid manipulation [117]. Plastics are also rapid and inexpensive to microfabricate [117]; their broad variety makes them more flexible for different applications [117]. Hydrogels are highly permeable, so they allow for diffusion of molecules without bulk fluid flows [117]; they are non-toxic to cells, cost effective and commercially available [118]. Paper also presents a lowcost, biocompatible, easy to use alternative, and it can be disposed after use [117]. Fluidic sample manipulation and biomarker detection can be performed in paper substrates thanks to the hydrophilicity of its fibers [119]; in addition, paper can be modified chemically to incorporate a wide variety of functional groups that can be covalently bound to proteins, DNA, or small molecules, and due to its white color it is a good medium for colorimetric tests, which makes 
them a good option for point-of-care applications [120]. One of the drawbacks of paper-based microfluidic devices is the complex patterning of the channels on the chip [118]. These materials can also be combined into hybrid chips in order to exploit their complementing advantages [117], [121].

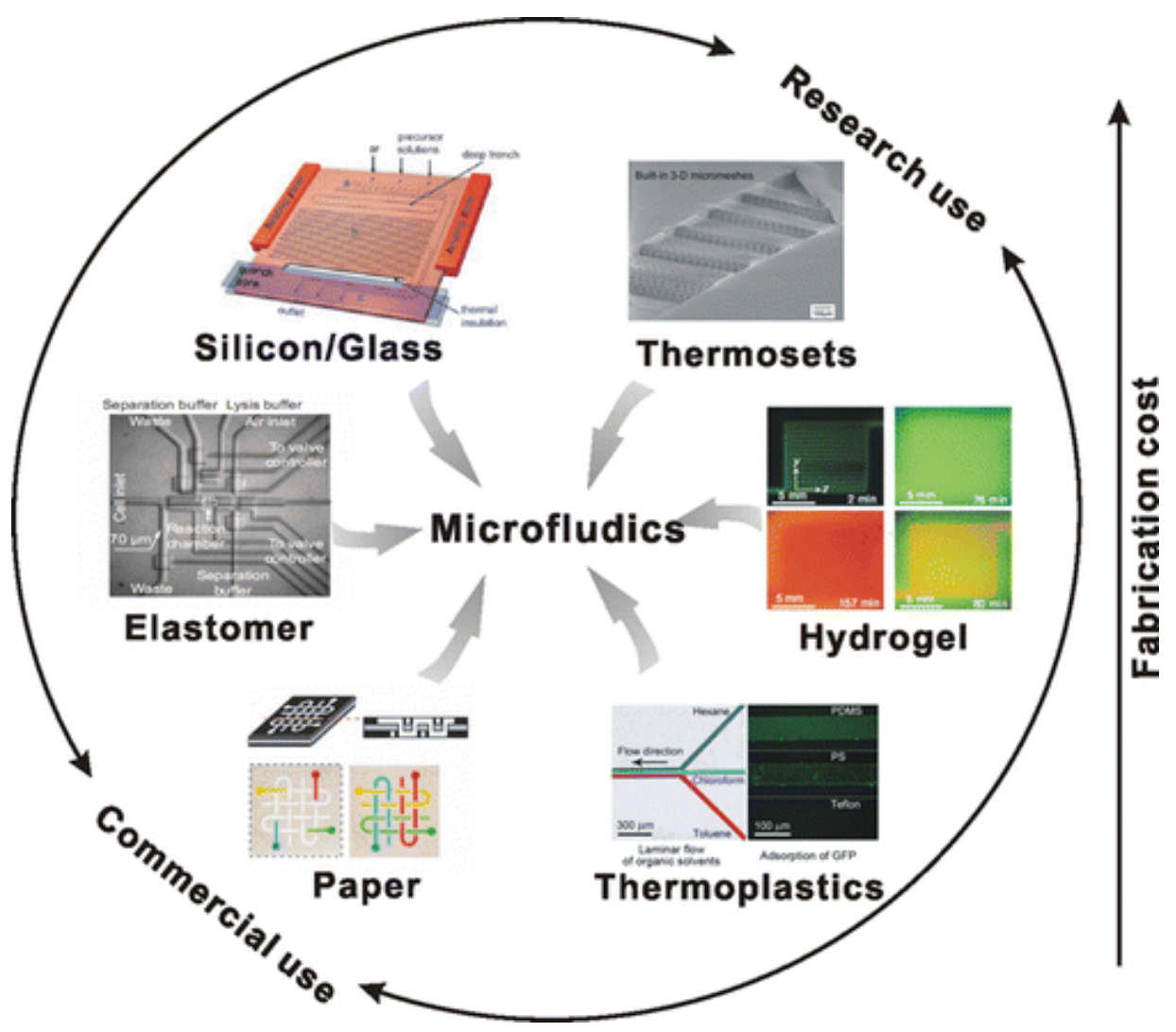

Figure 5-1 Microfluidics are fabricated in a variety of materials, including glass, silicon, paper, elastomers like PDMS, hydrogels, thermoplastics, and thermosets. Fabrication processes differ in degrees of complexity, and therefore in cost. The selection of the material to be used will depend on several factors, including the application, the target audience, and the fabrication capabilities [117].

\subsubsection{Capillary Systems Using Different Materials}

\section{Silicon-based Microfluidics}

One of the advantages of using silicon in microfluidics it's the capability to fabricate virtually any geometrical microstructure with high precision [122]. Silicon has high chemical 
resistance, mechanical strength, temperature stability, and it benefits from well-established MEMS fabricating and integration processes [123]. However, as previously mentioned, this material is not optically transparent in the UV/ visible region, limiting detection capabilities, and it is also very costly to fabricate, which greatly limits its usage in low-income settings [122]. In addition, Silicon has a hydrophobic nature, so it needs to undergo treatments in order to become hydrophilic (e.g. chemical treatment to make silicon surface $\mathrm{OH}$ terminated) [124]. A widely accepted procedure for rendering silicon wafers hydrophilic is the RCA cleaning [124], [125]; RCA consist of an aqueous solution of ammonia and hydrogen peroxide [124].

\section{Glass-based Microfluidics}

Glass has a good chemical resistance and it is very good for detection purposes thanks to its optical transparency, and its surface is hydrophilic in nature [122]. However, conventional glass capillary devices still have several limitations, including the complexity and cost of the fabrication methods [122], [126]. Recently, there has been some research on simplifying fabrication processes to overcome such limitations [126]-[128]. An example is the work of Benson et al., who developed a flow focusing glass capillary device based on commercially available chromatography components for easy assembly, disassembly, and cleaning [126].

\section{Paper-based Microfluidics}

Paper is an attractive substrate for microfluidic applications due to its availability and low cost, biocompatibility, and its ability to transport liquids using capillary forces, without the need of external equipment [129]. The first report on paper-based microfluidics might be the work of Müller et al. in 1949 [129]; their work consisted of creating confined channels by patterning filter paper using paraffin barriers, for the study on the preferential elution of a mixture of pigments [129], [130]. Paper microfluidics field has rapidly grown since then [129]. 
There are two types of "Lab-on-a-paper" devices for affordable disease diagnosis: The first consists of on demand devices, which are blank platforms that can be used for a variety of tests [129]. Upon the selection of the test to be performed, samples and reagents can be and added prior to the execution of the test [129]. The second consists of ready-to-use devices, which are test-specific, and already integrate the required reagents [129]. Most of the published works to date focus on the second type, since they are easier to handle by the patient [129]. Table 5-1 lists the different analytes studied to date using “Lab-on-a-paper”technologies [129].

Table 5-1 Summary of the reported detection methods, example analytes and applications for both paper-based microfluidic devices and paper-based microarray plates [129].

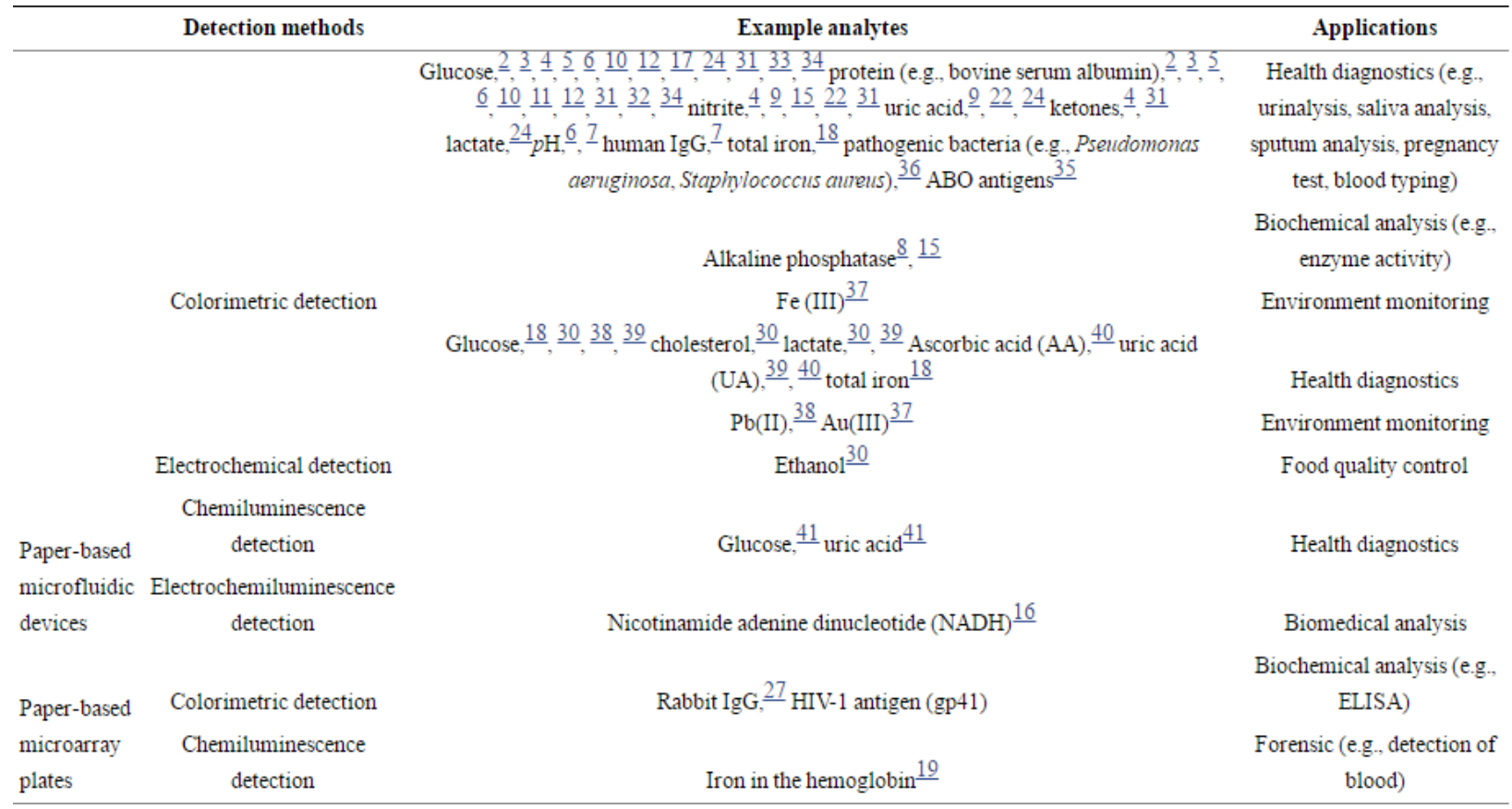

Although paper-based microfluidic devices have many advantages, they too have several limitations, which are related to the material properties of paper, fabrication techniques, and detection methodologies [129]. One of the limitations is sample retention and sample evaporation during fluid transport, which result in only $50 \%$ or less of the total volume reaching the detection zones [129]. Another limitation is the strength of the hydrophobic barriers, as some 
times it is not enough to withstand samples with low surface tension [129]. In addition, traditional detection methods have a high limit of the detection when integrated in paper substrates, making it problematic when analyzing samples of lower concentrations [129].

\section{Polymer-based Microfluidics}

Polymers are widely used in microfluidics, and they possess several advantages including biocompatibility, low cost (in comparison to glass and silicon), and ease of fabrication using a diversity of techniques, and bonding [131]. Their chemical resistance, optical transparency, low autofluorescence, and absence of UV absorption capabilities can be compared to those of glass [122]. The variety of surface chemistries also allows the usage of different immobilization techniques for the antibodies and/or antigens [122]. However, polymers often require surface functionalization in order to acquire certain properties such as hydrophilicity [132]. A key elastomer in microfluidics is PDMS, thanks to its relatively low cost and ease of fabrication, the capability of modifying its surface to render it hydrophilic, the ability to reversibly and irreversibly bond to other materials including glass, plastic, and other PDMS pieces, its elasticity, amongst others [133]. In addition, micro-scale features can be reproduced with high precision, it is optically transparent down to $280 \mathrm{~nm}$ so it can be used with a large number of detection methodologies, it is nontoxic and can be cured at low temperatures, and can be bonded reversibly or irreversibly [131]. However, PDMS also has some drawback such as its absorption of small molecules, the leaching of uncrosslinked oligomers, and the vapor permeability (evaporation effects can occur) [133]-[136]. Several surface modification methods have been used to overcome the hydrophobic nature limitation of PDMS [137]; such methods can be classified in three categories: gas-phase processing methods (e.g. plasma oxidation, ultraviolet (UV) irradiation, chemical vapor deposition (CVD) and sputter coating of metal compounds), 
wet chemical methods (e.g. layer-by-layer (LBL) deposition, sol--gel coatings, silanization, dynamic modification with surfactants and protein adsorption), and a combination of the previous [137].

In addition to PDMS, other polymeric materials have been used, including polycarbonate, polymethymethacrylate (PMMA), polystyrene, polypropylene, and PVC [122], [137].

\section{Hybrids}

There is no optimal material for all microfluidic applications. The selection depends on the specific application. In this work, we decided to take advantage of the capabilities of different materials in order to make an integrated hybrid capillary microfluidic platform combining PDMS polymer, paper, and glass. This allowed us to perform plasma separation from whole blood, antibody immobilization for detection in the near future, and flow initiation and control using capillary forces.

\subsubsection{Method}

Polydimethylsiloxane (PDMS) is a clear, colorless silicon-based organic polymer, and it is the most popular material for microfluidic research and prototyping due to its ease of fabrication, biocompatibility, nontoxicity, optical transparency, and gas permeability. However, because of its hydrophobic nature and strong adsorption of biological molecules such as proteins, PDMS is not suitable to be directly used to fabrication devices used in analytical, biological, and clinical applications. In this work, PDMS was functionalized with Pluronic F127 to modify its

hydrophobic surface for the LFD fabrication and operation. The molecules of pluronic have a triblock structure, consisting of one hydrophobic polypropylene oxide (PPO) group accompanied 
by two hydrophilic polyethylene oxide (PEO) units [138]. The general structure is $\mathrm{PEO}_{\mathrm{n}}-\mathrm{PPO}_{\mathrm{m}}-$ $\mathrm{PEO}_{\mathrm{n}}$, where $\mathrm{m}$ and $\mathrm{n}$ vary based on the type of pluronic (for pluronic $\mathrm{F} 127, \mathrm{~m}=100, \mathrm{n}=65$, molecular weight=12600) [138], [139]. The PPO chain attaches to the hydrophobic polymer (PDMS), while the PEO chains self-assemble into a cilia-like surface on the PDMS, effectively repelling proteins [138], [140]. Pluronic copolymers are an important type of surfactants due to their low toxicity and immunogenic response; they are approved for medical applications by the US Food and Drug administration [138], [140].

The microchannels networks were fabricated using standard photolithography and soft lithography techniques [141]. The process consisted of utilizing a high-resolution photomask to expose and polymerize a polymer-based photoresist spun cast onto a silicon wafer [6]. Following development, the resulting three-dimensional (3D) structure was used as a template for replication in Pluronic F127 (Sigma-Aldrich Co.) functionalized PDMS (Sylgard 184). The 5 $\mathrm{mm}$ by $10 \mathrm{~mm}$ glass chips where cut using a diamond tip, and the interfacing layer was then fabricated by placing the chips on a $10.16 \mathrm{~cm}$ by $10.16 \mathrm{~cm}$ petri dish, and casting the Pluronic functionalized PDMS on the dish, followed by degassing, and curing 1 hour at $60{ }^{\circ} \mathrm{C}$. The result consisted of a clean, flat Pluronic functionalized PDMS surface, with the glass chip embedded in it, which was then bonded to the PDMS replica (flow layer) to generate a sealed microfluidic network.

SU-8 2025 negative photoresist (Microchem) was used for fabricating the fluidic layer mold. SU-8 2025 was spin coated on a silicon wafer to a thickness of $20 \mu \mathrm{m}$, exposed to UV radiation, and rinsed in SU-8 developing solution to create the microchannel templates. Channel height was then measured using a Stylus Profilometer. 
$200 \mathrm{mg}$ of Pluronic F127 were dissolved in $1 \mathrm{~mL}$ of alcohol. Samples with $20 \mu \mathrm{L}$ and $40 \mu \mathrm{L}$ of this complex were mixed thoroughly with the freshly made uncured $10 \mathrm{~g}$ PDMS, resulting the final Pluronic concentration as $2 \mu \mathrm{L} / \mathrm{g}$ and $4 \mu \mathrm{L} / \mathrm{g}$, respectively. The PDMS/Pluronic mixed solution was then cast over the SU-8 master mold creating an inverse replica of the design, and cured for one hour at $60{ }^{\circ} \mathrm{C}$. Once cured, the PDMS/Pluronic layer was removed from the master mold. Inlets and outlets for the fluids were cut out from the interfacing layer using a sharpened puncher (Figure 5-2).

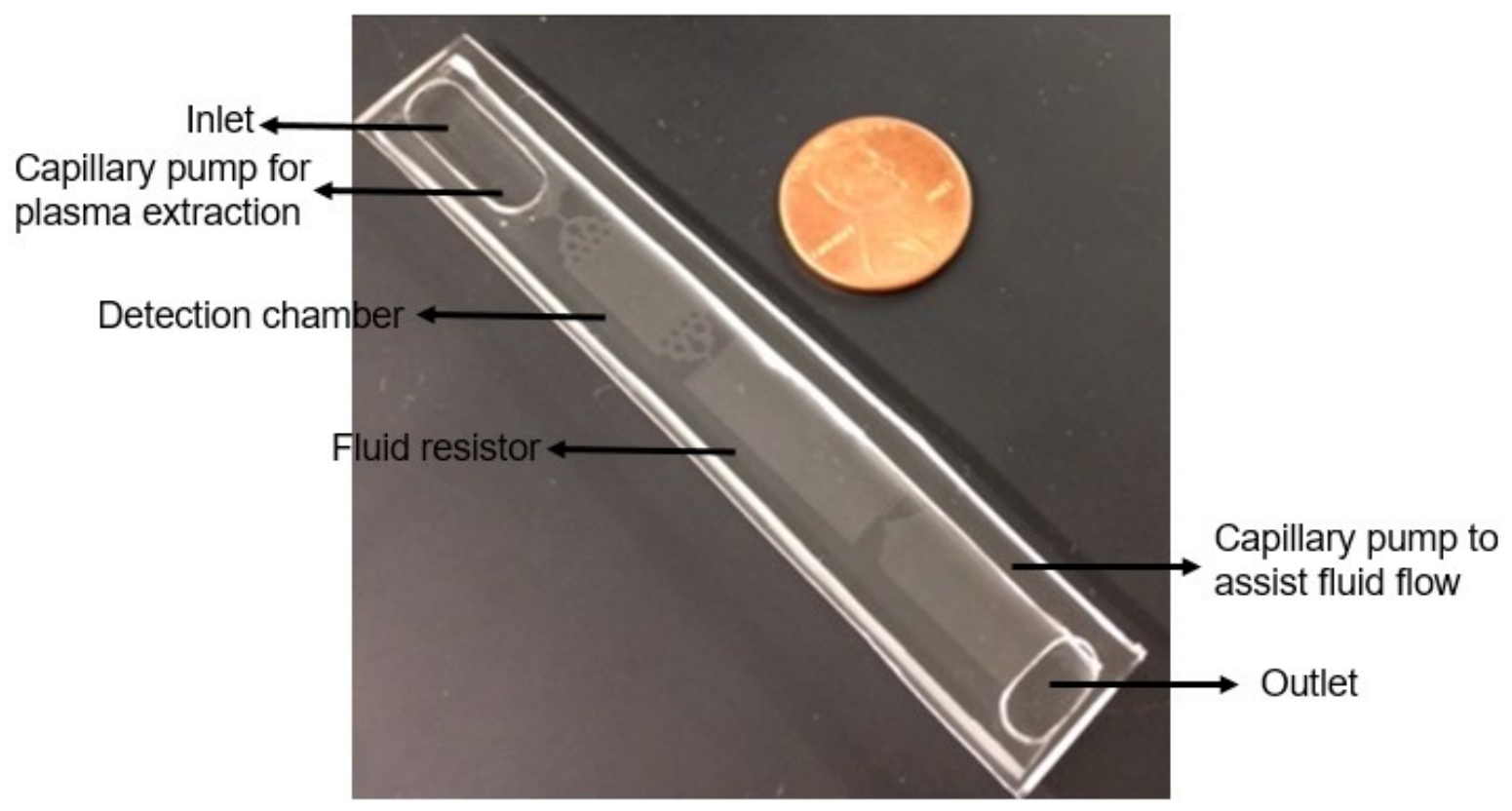

Figure 5-2 Photograph of the fluidic microchannels

In aqueous environments, the Pluronic migrates towards the surface due to the high gradient between the water and the PDMS substrate, resulting in a reduction of nonspecific adsorption and an enhancement of wetting [138], [139]; for this reason, both layers were immersed in 500 $\mathrm{mL}$ de-ionized water for $24 \mathrm{~h}$ at room temperature. After the $24 \mathrm{~h}$ immersion, the surfaces of both the flow and the interfacing Pluronic functionalized PDMS layers were dried with nitrogen gas and then exposed to oxygen plasma treatment for 30 seconds. The flow layer was immediately placed in conformal contact with the interfacing layer, irreversibly bonding them 
together, ensuring that the glass chip on the interfacing layer was placed directly on top of the detection chamber on the flow layer, and then stabilized in an oven at $60{ }^{\circ} \mathrm{C}$ for half an hour.

\subsection{Flow Rate Control}

\subsubsection{Background}

Flow control is a key in the proper functioning of microfluidics devices, and it can be achieved by using a combination of valves, pumps, and resistances [3]. The overall flow rate of the fluid is encoded by the pumping mechanism, which can be either active (e.g. reciprocating movement membrane valve, capillary electrophoresis using electro-osmotic flow, centrifugal force in Lab on a CD devices, electro wetting) or passive (e.g. porous capillary membrane, capillary pump, vacuum driven suction) depending on the application requirements [3], [142].

\subsubsection{Active Pumping}

These type of microfluidic systems require external macroscopic actuators such as syringe pumps, peristaltic pumps, air pressure pumps, multiport valves, sensors, and even computers for flow rate control [168], [178]-[180]. Flow can be driven by mechanical displacement, electrical fields, magnetic fields or centripetal force [3].

Active pumping setups provide a higher degree of interactive control, and the allow for controlling liquids in a broad range of flow rates, pressures, viscosities, molecular weights and compositions, but at the expense of complexity, lack of portability, scaling, and cost [3], [4]; for this reason their main application is in research settings [3].

Syringe pumps are frequently used in microfluidics due to their ease of use [3]. The working mechanism consists of a stepper motor which causes the syringe's plunger to move at a 
constant speed, encoding the desired flow rate, while small tubing connect the syringe to the microfluidic chip [3]. Microfabricated mechanical displacement pumps actuate a membrane or diaphragm in a reciprocating mode [3], [143], [144].

Electro-osmotic pumping is based on a chemical equilibrium at the interface between a liquid and a solid, resulting in a charged surface and counter ions in the liquid [3]. When applying an electrical current, the counter ions will move with the electrical current, creating a pressure gradient and therefore flow of the liquid [3]. However, electro-osmotic pumping has some weaknesses, including the sensitivity to impurities that adsorb on the wall of the channel and to changes in composition of the buffer, ohmic generation of heat in the fluid, and the need for very high voltages and therefore macroscopic power supplies [4].

Another approach is the lab on a CD, where the liquid is loaded into a reservoir near to the disk's center, and when the rotated at varying speeds, the liquid is pumped through the microfluidic channels thanks to centrifugal force [3].

Electrowetting devices apply pulses of electrical potentials between ground and actuation electrodes creating electrostatic and dielectrophoresis forces that temporarily change the wetting properties, rendering the surface hydrophilic, which allows for fluid flow [3].

Several alternate active pumping schemes has been reported in scientific literature [4].

\subsubsection{Passive Pumping}

Flow rates in passive pumping are a function of the design. Passive microfluidics uses chemical gradients on surfaces, osmotic pressure, permeation in PDMS, or capillary forces to drive the fluid through the microfluidic channels [3]. These systems have spontaneous filling and 
present an attractive alternative due to their portability, zero power consumption, low volume requirement, low cost, and ease of handling [3]. However, these devices offer less flexibility of modification by the user.

In capillary systems (CS), liquids are displaced by means of capillarity. Their proper functioning requires displacing accurate volumes of liquids with precise flow rates [98]. The capillary pressure is a function of the depth and width of the microchannels, the surface tension $(\gamma)$ and contact angles with the bottom, top, left and right side walls [3]. The capillary resistance is a function of the geometry of the channel, and together with capillary pressure and viscosity of the fluid $(\eta)$, it can aid in calculating the flow rate [3]. Capillary pumps (CPs) encompass micro pillars of diverse shapes and dimensions, whose position in the capillary pump (CP) encodes the desired capillary pressure [98], and together with their shape, will determine the filling front of the liquid in the CP as well as the flow rate [98]. The high differential pressures created by the CPs allow for supplying near-constant continuous flow over the course of several minutes [8]. Berthier et al. investigated a microfluidic passive pumping method relying on surface tension, in which a small inlet drop was placed on the entrance of a microfluidic channel, creating higher pressure than that of a larger output drop at the channel exit, resulting in the flow of the fluid [145]. Other methods require an additional user induced pressure (manual pressure), such is the case of the control scheme developed by Vestad et al. by which capillary networks flow could be dynamically controlled through simple external pressure and membrane deflection [6]. Sometimes controlling more than one working fluid is necessary, for example for multiplexing biomarker detection or parallelization of miniaturized bio/chemical processes [9]; Junker et al. developed a CS that could autonomously transport aliquots of different liquids in sequence [9]. 
Capillary driven microfluidics systems are low cost, easy to handle, and portable, so they are a good alternative for POC applications, however, they do present some challenges such as evaporation of liquid in loading pads, blocked channel due to debris or defects, and higher resistance with lower dimensions, which can be addressed with a proper design and manufacturing process [3].

\subsubsection{Contact Angle Measurement}

To understand the effect of resistance variation, and Pluronic concentration in the PDMS, and verify the hydrophilicity of the surface, the static and dynamic contact angles of several samples were measured. Concentrations of $2 \mu \mathrm{L} / \mathrm{g}$ and $4 \mu \mathrm{L} / \mathrm{g}$ were taken into account (concentrations lower than $2 \mu \mathrm{L} / \mathrm{g}$ were also studied but the contact angle variation was very small, while concentrations higher than $4 \mu \mathrm{L} / \mathrm{g}$ turned out too cloudy, which could conflict with optical detection methods). Because contact angles on the channel wall surfaces may be significantly different from smooth and flat surfaces [146], both the static and in-situ dynamic contact angles were measured and compared to study the wettability of PDMS surfaces with/without Pluronic functionalization. Static contact angle on the flat surface was measured using a handheld digital microscope (Celestron, LLC), by placing the microscope in horizontal position (parallel to the substrate), such that an image of the droplet outline and its reflection on the substrate could be taken; static contact angles were analyzed using Image J software (NIH). For the dynamic contact angle measurement, a drop of liquid $(40 \mu \mathrm{L})$ was placed in the inlet port of the LFD, and the air-liquid interface was recorded by performing a time-lapse imaging using a Nikon Eclipse Ti microscope, using objective lenses with a magnification from $4 \times$ to $20 \times$. NISElements Viewer (Nikon Instruments Inc.) was then used for analysis. Experiments were 
performed for both PBS and Human Blood plasma, on both water treated and Pluronic functionalized PDMS without water treatment.

In addition, the dynamic contact angles were measured for all five flow resistors, in order to evaluate the relationship between the resistance and contact angle. For statistical analysis, data was presented as the mean \pm standard derivation (SD) and each individual measurement/experiment was performed at least three times $(n>3)$.

Note that in this work we describe two types of fluidic resistances. The first, total flowrate resistance is function of the geometry of the microchannels alone (Equation 4); while the second, hydraulic resistance, accounts for not only the geometric parameters but also the viscosity of the fluid flowing through the channels (Equation 10). Since the total flow-rate is only function of geometry and is independent of viscosity, its magnitude is equivalent for both PBS and Plasma for each individual flow resistor (Table 5-2). On the other hand, the magnitude of the Hydraulic resistance is always higher for plasma since its viscosity is greater than that of PBS (Table 5-2).

Table 5-2 Total flow-rate resistance vs. Hydraulic resistance PBS

\begin{tabular}{|l|l|l|l|l|l|l|}
\hline \multicolumn{2}{|l|}{ Channel geomesistance $\left(\mathrm{m}^{-3}\right)$} & \multicolumn{3}{l|}{ Hydraulic resistance $\left(\mathrm{kg}^{*} \mathrm{~m}^{-4} *^{-1}\right)$} \\
\hline $\begin{array}{l}\text { Length of } \\
\text { microchannel } \\
(\mathrm{cm})\end{array}$ & $\begin{array}{l}\text { Depth of the } \\
\text { microchannel } \\
(\mu \mathrm{m})\end{array}$ & $\begin{array}{l}\text { Width of the } \\
\text { microchannel } \\
(\mu \mathrm{m})\end{array}$ & PBS & Plasma & PBS & Plasma \\
\hline 0.324 & 28.4 & 200 & $\begin{array}{l}9.90 \mathrm{E}+15 \\
\text { (no flow } \\
\text { resistor) }\end{array}$ & $\begin{array}{l}\text { 9.90E+15 } \\
\text { (no flow } \\
\text { resistor) }\end{array}$ & $\begin{array}{l}\text { 9.689E+12 } \\
\text { (no flow } \\
\text { resistor) }\end{array}$ & $\begin{array}{l}1.54659 \mathrm{E}+13 \\
\text { (no flow } \\
\text { resistor) }\end{array}$ \\
\hline 3 & 27.3 & 200 & $1.02 \mathrm{E}+17$ & $1.02 \mathrm{E}+17$ & $1.01 \mathrm{E}+14$ & $1.61 \mathrm{E}+14$ \\
\hline 10 & 25.1 & 200 & $4.35 \mathrm{E}+17$ & $4.35 \mathrm{E}+17$ & $4.28 \mathrm{E}+14$ & $6.84 \mathrm{E}+14$ \\
\hline 20 & 25 & 200 & $8.80 \mathrm{E}+17$ & $8.80 \mathrm{E}+17$ & $8.67 \mathrm{E}+14$ & $1.38 \mathrm{E}+15$ \\
\hline 37 & 22 & 200 & $2.35 \mathrm{E}+18$ & $2.35 \mathrm{E}+18$ & $2.33 \mathrm{E}+15$ & $3.72 \mathrm{E}+15$ \\
\hline
\end{tabular}




\subsubsection{Results}

\subsubsection{Dynamic vs. Static Contact Angle Measurement}

Due to the hydrophobic nature of the PDMS, surface modification via functionalization with Pluronic F127 was necessary. The effects of the surface modification and the variation of the flow resistor's resistance on the characteristics of the capillary flow were studied in five different microfluidic devices. Table 5-3 presents a summary of the parameters measured in this work.

Table 5-3 Summary of measured parameters

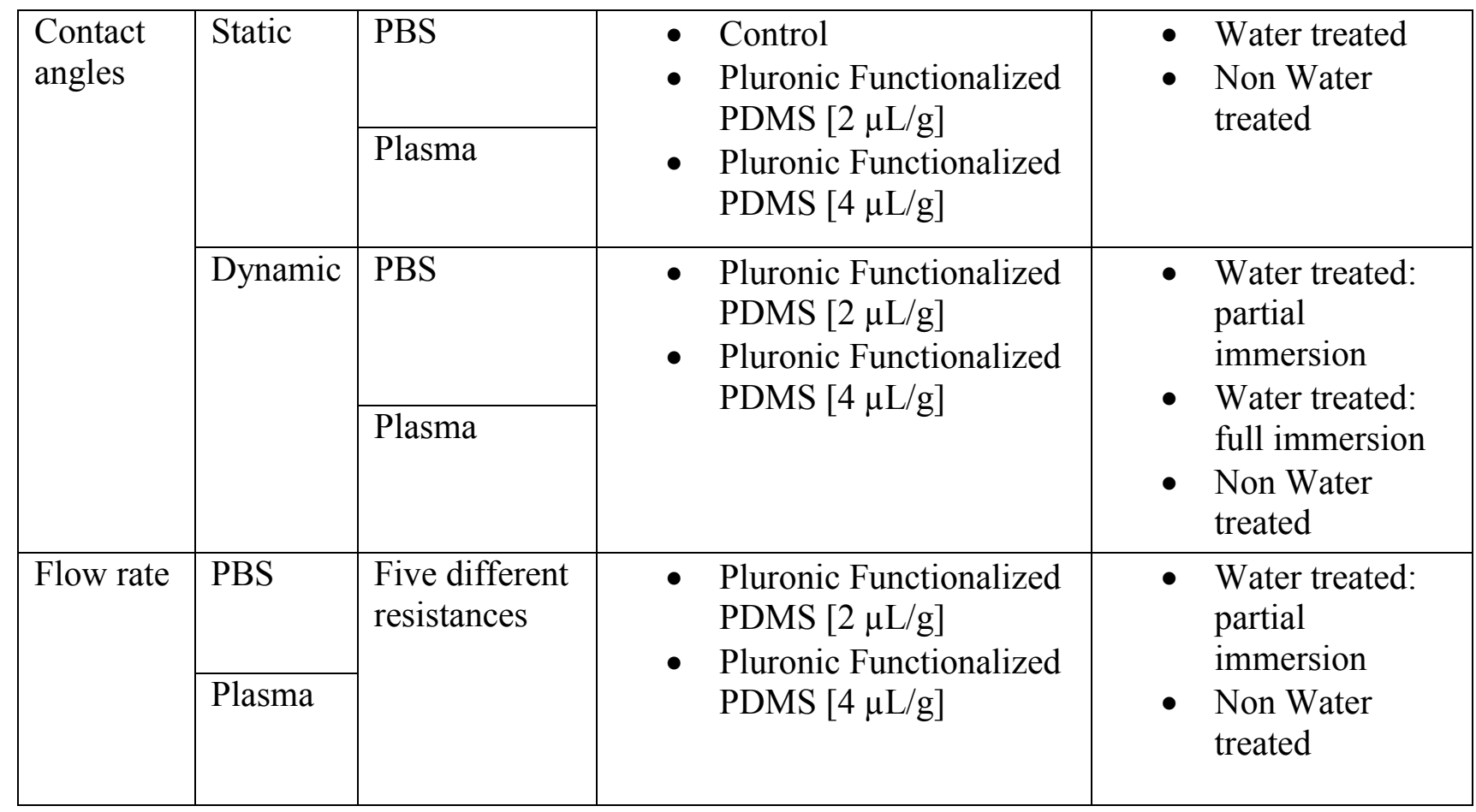

Static contact angles measured from smooth flat surfaces can greatly differ from in situ dynamic contact angles, and they do not account for the resistance encoded by the constricted microchannel and the structures in the capillary pumps (influenced by their shape, size, and spacing between structures). Static contact angles were measured by dispensing a droplet of 
Plasma or PBS on the surface of the Pluronic functionalized PDMS (without water exposure). The angles were continuously monitored for a period of 20 minutes (Figure 5-3) [147]. As a point of comparison, dynamic contact angles were measured using Nikon Eclipse Ti microscope. For the flow resistor, dynamic contact angle measurements were always taken at the entrance channels for consistency. The inverted microscopy technique allowed for measuring only the top and bottom dynamic contact angles $\left(\alpha_{b}\right.$ and $\left.\alpha_{t}\right)$, however, side angles can be calculated using Equation 6. Static contact angle measurements where used in the flow equations in order to compare the theoretical and the experimental flow rate.
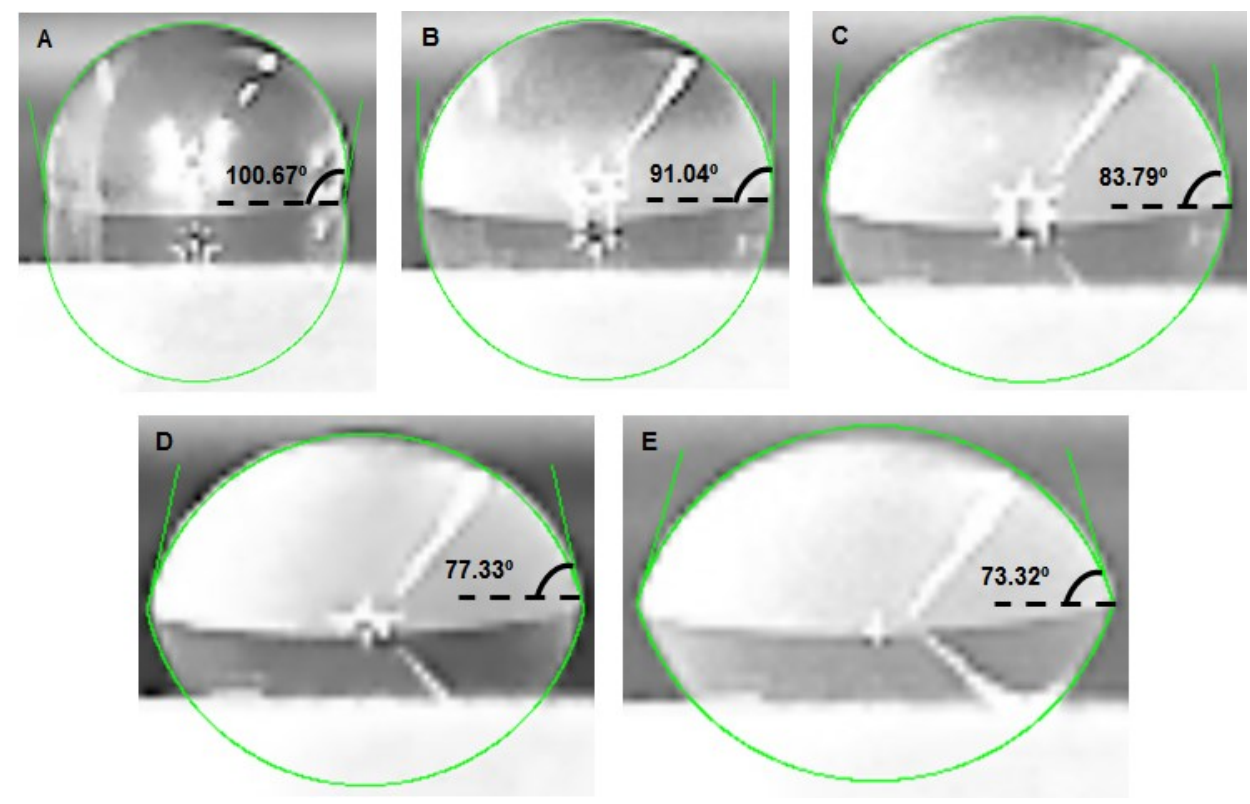

Figure 5-3 Representative images showing the change of the static contact angles of a $10 \mu \mathrm{L}$ droplet of plasma Pluronic functionalized PDMS $[4 \mu \mathrm{L} / \mathrm{g}]$. A) Initial contact angle just after dispensing the droplet. B) 5 min after dispensing the droplet. C) 10 min after dispensing the droplet. D) $15 \mathrm{~min}$ after dispensing the droplet. E) $20 \mathrm{~min}$ after dispensing the droplet.

Table 5-4 Static contact angle without water treatment

\begin{tabular}{|l|l|l|l|}
\hline & \multicolumn{2}{|l|}{ Plasma } \\
\hline & Control & $\begin{array}{l}\text { Pluronic/PDMS } \\
{[2 \mu \mathrm{L} / \mathrm{g}]}\end{array}$ & $\begin{array}{l}\text { Pluronic/PDMS } \\
{[4 \mu \mathrm{L} / \mathrm{g}]}\end{array}$ \\
\hline Static contact angle $($ mean \pm SD) & $79.82^{\circ} \pm 3.91^{\circ}$ & $68.42^{\circ} \pm 5.22^{\circ}$ & $69.85^{\circ} \pm 1.30^{\circ}$ \\
\hline
\end{tabular}


Static contact angles were measured for a continuous period of 20 minutes as to provide enough time for the droplet contact angle to stabilize [147]. The angles where originally hydrophobic, and the change from hydrophobic to hydrophilic occurred after 5 minutes [147]. Table 5-4 shows the magnitude of the stabilized static contact angle after 20 minutes. For the pluronic functionalized samples, the concentration of pluronic had a very small impact on the static contact angle.

On the other hand, the pluronic concentration had a greater effect on the dynamic contact angle (Figures 5-4B and 5-4C). Dynamic contact angles proved more hydrophilic with increase in the concentration, however, the flow resistance proved the mayor influence in both the magnitude of the contact angles, and more importantly, the flow rate. The contact angle was measured for sets of identical microchannels, with the only difference being the concentration of the Pluronic in the PDMS. The contact angle proved to be reduced as concentration increased (Table 5-5). It is known that the pillar aspect ratio and their positioning in the capillary pumps, as well as and the physical properties of the fluid (e.g. surface tension, viscosity), have a great impact on the capillary phenomena in the microchannel [148], [149]; with this in mind contact angles were also measured in both the first and second capillary pumps. In a similar way to the flow resistor's contact angles, the capillary pump's contact angles had a tendency to decrease with higher concentrations of pluronic (Tables 5-6 and 5-7). The effect of the resistance, determined by the microstructures in the capillary pump, on the magnitude of the contact angle can be observed in Figure 5-5. The contact angle proved more hydrophilic for the CP2 than those of the CP1. 

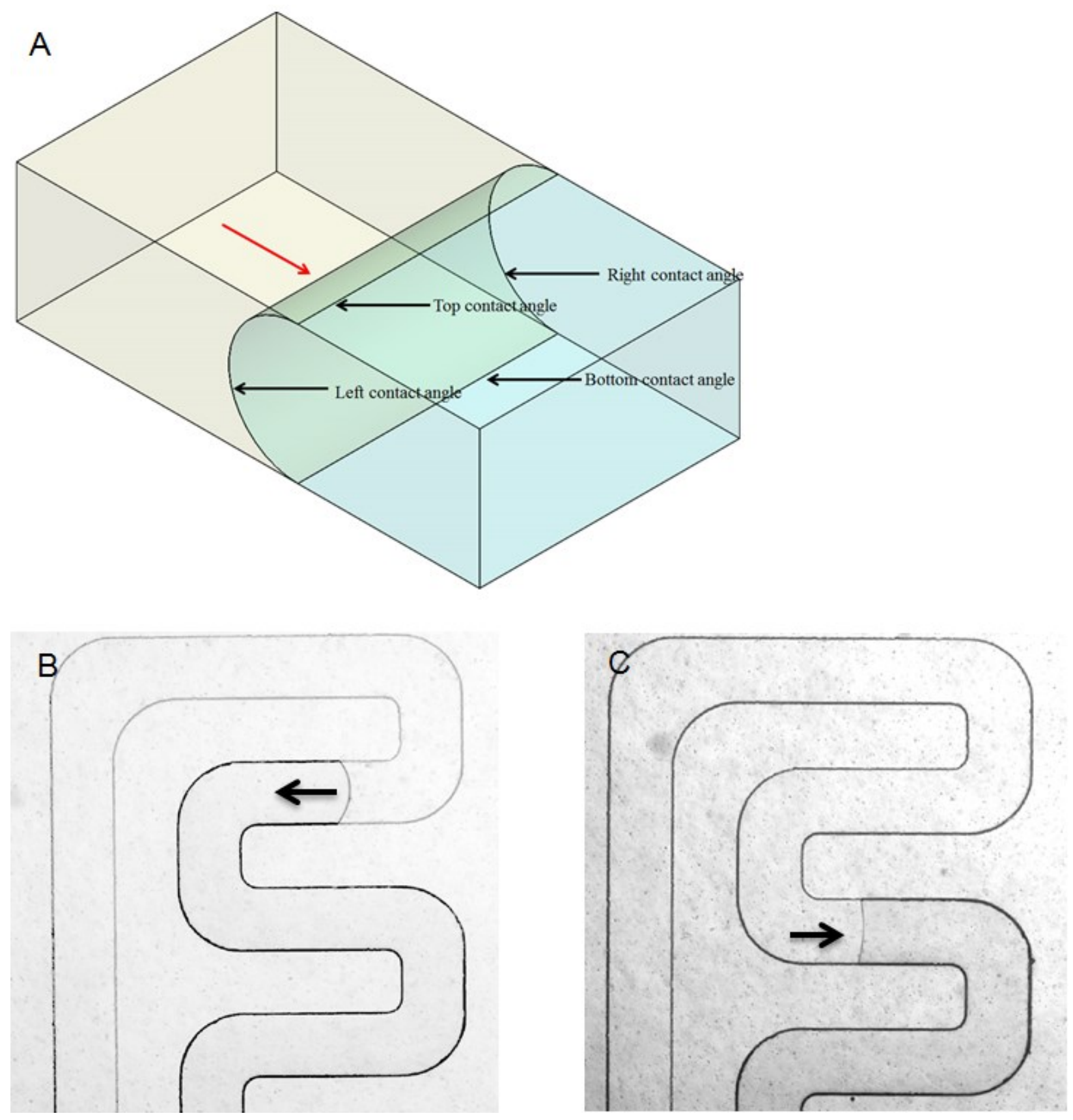

Figure 5-4 A) Schematic of Dynamic contact angle in Flow Resistors. The interface has concave contact lines for the side walls and nearly flat $\left(90^{\circ}\right)$ contact lines for the top and bottom walls; the contact lines with the top and bottom walls can also have a convex shape when the contact angle is greater than $90^{\circ}$. The light area represents the plasma phase, which moves from left to right. B) Highly hydrophilic microchannel (Pluronic/PDMS [4 $\mu \mathrm{L} / \mathrm{g}]$ ). C) Less hydrophilic microchannel (Pluronic/PDMS $[2 \mu \mathrm{L} / \mathrm{g}]$ ). The black arrows indicated the flow direction in the microchannel. 

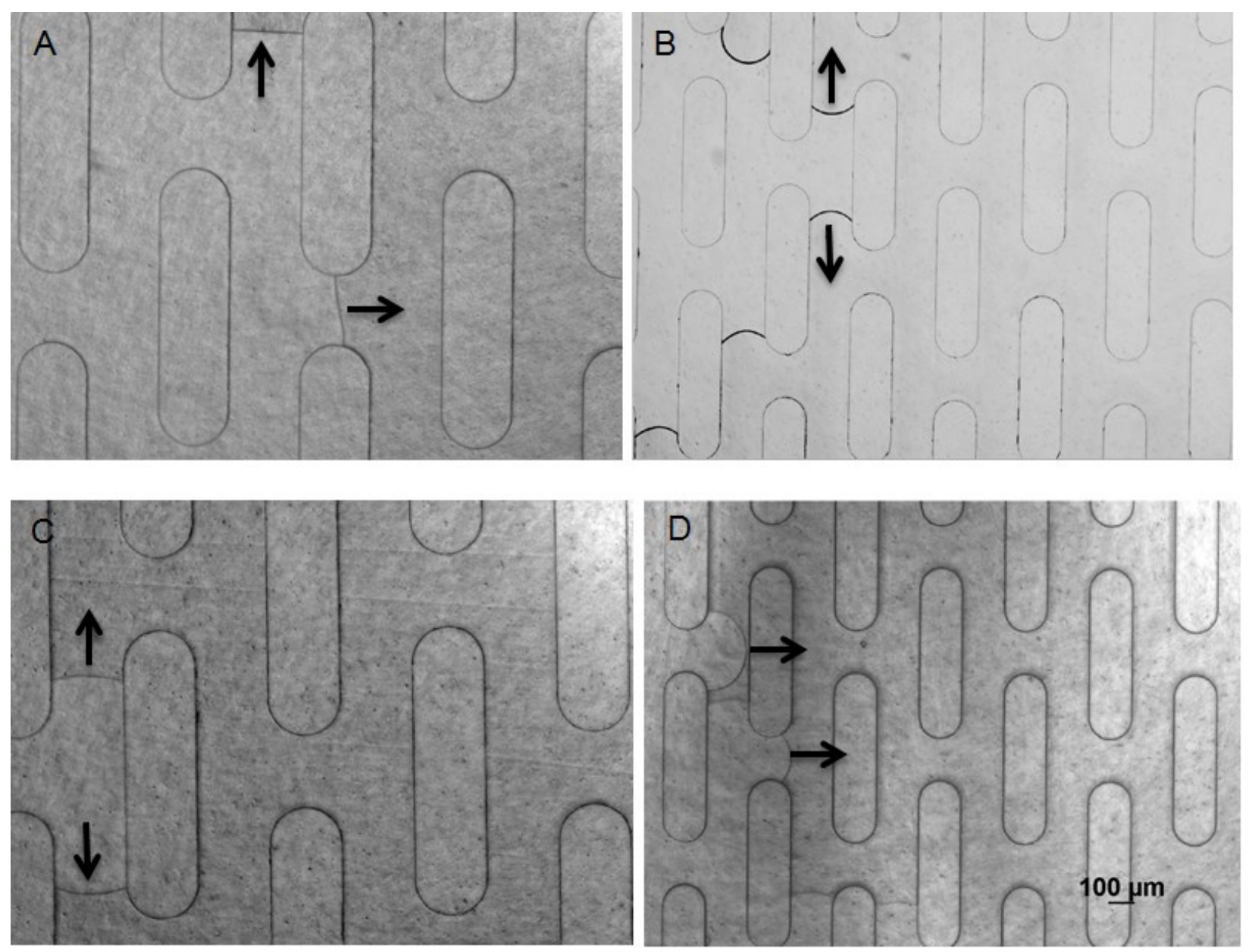

Figure 5-5 Representative results showing the dynamic contact angles in the capillary pumps. A) CP1, Plasma, Pluronic functionalized PDMS (Pluronic/PDMS $[4 \mu \mathrm{L} / \mathrm{g}]$ ). B) CP2, plasma, Pluronic functionalized PDMS (Pluronic/PDMS [4 $\mu \mathrm{L} / \mathrm{g}]$ ). C) CP1, PBS, Pluronic functionalized PDMS (Pluronic/PDMS [2 $\mu \mathrm{L} / \mathrm{g}]$ ). D) CP2, Plasma, Pluronic functionalized PDMS (Pluronic/PDMS $[2 \mu \mathrm{L} / \mathrm{g}]$ ). The black arrows indicated the flow direction in the capillary pumps.

Tables 5-5, 5-6, and 5-7 show that $\alpha_{\mathrm{b}}$ and $\alpha_{\mathrm{t}}$ measurements often tend to be close to $90^{\circ}$ or larger; this occurs because the dynamic contact angle tends to increase rapidly in response to increased wetting speed [146], [150]. If the dynamic angles of the left and right side walls $\left(\alpha_{1}\right.$ and $\alpha_{\mathrm{r}}$ ) were assumed to be equal to $\alpha_{\mathrm{b}}$ and $\alpha_{\mathrm{t}}$, the capillary pressure would be zero or negative, resulting in a stationary interface or the interface moving in the opposite direction, which would contradict the observed experimental results [146]. The fact that the meniscus moved in the 
positive implies that $\alpha_{1}$ and $\alpha_{\mathrm{r}}$ are smaller than $90^{\circ}$ [146]. Figure 5-4A shows the threedimensional schematic of the interface in the functionalized channels.

Table 5-5 Dynamic contact angle in flow resistor (Top/Bottom)

\begin{tabular}{|l|l|l|l|l|}
\hline $\begin{array}{l}\text { Flow resistance } \\
\left(\mathrm{m}^{-3}\right)\end{array}$ & PBS & \multicolumn{3}{|l|}{ Plasma } \\
\cline { 2 - 5 } & $\begin{array}{l}\text { Pluronic/ } \\
\text { PDMS } \\
{[2 \mu \mathrm{L} / \mathrm{g}]} \\
\text { mean } \pm \mathrm{SD}\end{array}$ & $\begin{array}{l}\text { Pluronic/ } \\
\text { PDMS } \\
{[4 \mu \mathrm{L} / \mathrm{g}]} \\
\text { mean } \pm \mathrm{SD}\end{array}$ & $\begin{array}{l}\text { Pluronic/ } \\
\text { PDMS } \\
{[2 \mu \mathrm{L} / \mathrm{g}]} \\
\text { mean } \pm \mathrm{SD}\end{array}$ & $\begin{array}{l}\text { Pluronic/ } \\
\text { PDMS } \\
{[4 \mu \mathrm{L} / \mathrm{g}]} \\
\text { mean } \pm \mathrm{SD}\end{array}$ \\
\hline $\begin{array}{l}9.90 \mathrm{E}+15 \\
\text { (no flow resistor) }\end{array}$ & $91.80^{\circ} \pm 1.83^{\circ}$ & $101.75^{\circ} \pm 8.03^{\circ}$ & $105.60^{\circ} \pm 3.07^{\circ}$ & $102.47^{\circ} \pm 2.54^{\circ}$ \\
\hline $1.02 \mathrm{E}+17$ & $99.43^{\circ} \pm 3.50^{\circ}$ & $94.30^{\circ} \pm 5.21^{\circ}$ & $106.80^{\circ} \pm 4.66^{\circ}$ & $73.58^{\circ} \pm 15.67^{\circ}$ \\
\hline $4.35 \mathrm{E}+17$ & $91.87^{\circ} \pm 1.96^{\circ}$ & $88.60^{\circ} \pm 11.78^{\circ}$ & $104.00^{\circ} \pm 1.00^{\circ}$ & $58.90^{\circ} \pm 4.96^{\circ}$ \\
\hline $8.80 \mathrm{E}+17$ & $101.00^{\circ} \pm 1.31^{\circ}$ & $63.25^{\circ} \pm 4.90^{\circ}$ & $108.26^{\circ} \pm 3.31^{\circ}$ & $95.45^{\circ} \pm 2.85^{\circ}$ \\
\hline $2.35 \mathrm{E}+18$ & $107.00^{\circ} \pm 8.07^{\circ}$ & $96.00^{\circ} \pm 8.48^{\circ}$ & $94.00^{\circ} \pm 3.67^{\circ}$ & $91.30^{\circ} \pm 1.67^{\circ}$ \\
\hline
\end{tabular}

Table 5-6 Dynamic contact angle in cp1 (Top/Bottom)

\begin{tabular}{|c|c|c|c|c|}
\hline \multirow{3}{*}{\begin{tabular}{|l} 
Flow \\
resistance \\
$\left(\mathrm{m}^{-3}\right)$
\end{tabular}} & \multicolumn{2}{|l|}{ PBS } & \multicolumn{2}{|l|}{ Plasma } \\
\hline & $\begin{array}{l}\text { Pluronic/ } \\
\text { PDMS } \\
{[2 \mu \mathrm{L} / \mathrm{g}]} \\
\text { mean } \pm \mathrm{SD}\end{array}$ & $\begin{array}{l}\text { Pluronic/ } \\
\text { PDMS } \\
{[4 \mu \mathrm{L} / \mathrm{g}]} \\
\text { mean } \pm \mathrm{SD}\end{array}$ & $\begin{array}{l}\text { Pluronic/ } \\
\text { PDMS } \\
{[2 \mu \mathrm{L} / \mathrm{g}]} \\
\text { mean } \pm \text { SD }\end{array}$ & $\begin{array}{l}\text { Pluronic/ } \\
\text { PDMS } \\
{[4 \mu \mathrm{L} / \mathrm{g}]} \\
\text { mean } \pm \mathrm{SD}\end{array}$ \\
\hline & $\begin{array}{l}\text { Top/Bottom } \\
\text { Contact Angle }\end{array}$ & $\begin{array}{l}\text { Top/Bottom } \\
\text { Contact Angle }\end{array}$ & $\begin{array}{l}\text { Top/Bottom } \\
\text { Contact Angle }\end{array}$ & $\begin{array}{l}\text { Top/Bottom } \\
\text { Contact Angle }\end{array}$ \\
\hline $7.73 \mathrm{E}+15$ & $98.17^{\circ} \pm 2.40^{\circ}$ & $107.89^{\circ} \pm 3.14^{\circ}$ & $107.50^{\circ} \pm 6.40^{\circ}$ & $96.75^{\circ} \pm 5.06^{\circ}$ \\
\hline $8.69 \mathrm{E}+15$ & $104.50^{\circ} \pm 2.52^{\circ}$ & $94.75^{\circ} \pm 2.75^{\circ}$ & $117.20^{\circ} \pm 7.08^{\circ}$ & $91.50^{\circ} \pm 0.58^{\circ}$ \\
\hline $1.11 \mathrm{E}+16$ & $95.75^{\circ} \pm 2.99^{\circ}$ & $90.42^{\circ} \pm 1.36^{\circ}$ & $97.50^{\circ} \pm 1.97$ & $100.00^{\circ} \pm 2.21^{\circ}$ \\
\hline $1.13 \mathrm{E}+16$ & $103.50^{\circ} \pm 2.08^{\circ}$ & $87.62^{\circ} \pm 2.43^{\circ}$ & $109.33^{\circ} \pm 6.03^{\circ}$ & $101.17^{\circ} \pm 5.60^{\circ}$ \\
\hline $1.64 \mathrm{E}+16$ & $104.06^{\circ} \pm 1.93^{\circ}$ & $80.28^{\circ} \pm 2.87^{\circ}$ & $96.62^{\circ} \pm 2.87^{\circ}$ & $108.00^{\circ}$ \\
\hline
\end{tabular}

The left and right contact angles can be estimated by calculating the experimental flow rate for each component and using the equations in Section 3.1.2 to find the $\alpha_{1}$ and $\alpha_{\mathrm{r}}$ that would encode that flow rate. 
Table 5-7 Dynamic contact angle in cp2 (Top/Bottom)

\begin{tabular}{|c|c|c|c|c|}
\hline \multirow{3}{*}{$\begin{array}{l}\text { Flow } \\
\text { resistance } \\
\left(\mathrm{m}^{-3}\right)\end{array}$} & \multicolumn{2}{|l|}{ PBS } & \multicolumn{2}{|l|}{ Plasma } \\
\hline & $\begin{array}{l}\text { Pluronic/ } \\
\text { PDMS } \\
{[2 \mu \mathrm{L} / \mathrm{g}]} \\
\text { mean } \pm \mathrm{SD}\end{array}$ & $\begin{array}{l}\text { Pluronic/ } \\
\text { PDMS } \\
{[4 \mu \mathrm{L} / \mathrm{g}]} \\
\text { mean } \pm \mathrm{SD}\end{array}$ & $\begin{array}{l}\text { Pluronic/ } \\
\text { PDMS } \\
{[2 \mu \mathrm{L} / \mathrm{g}]} \\
\text { mean } \pm \mathrm{SD}\end{array}$ & $\begin{array}{l}\text { Pluronic/ } \\
\text { PDMS } \\
{[4 \mu \mathrm{L} / \mathrm{g}]} \\
\text { mean } \pm \mathrm{SD}\end{array}$ \\
\hline & $\begin{array}{l}\text { Top/Bottom } \\
\text { Contact Angle }\end{array}$ & $\begin{array}{l}\text { Top/Bottom } \\
\text { Contact Angle }\end{array}$ & $\begin{array}{l}\text { Top/Bottom } \\
\text { Contact Angle }\end{array}$ & $\begin{array}{l}\text { Top/Bottom } \\
\text { Contact Angle }\end{array}$ \\
\hline $3.17 \mathrm{E}+16$ & $93.67^{\circ} \pm 1.53^{\circ}$ & $93.06^{\circ} \pm 1.86^{\circ}$ & $95.12^{\circ} \pm 4.87^{\circ}$ & $103.00^{\circ} \pm 4.24^{\circ}$ \\
\hline $3.56 \mathrm{E}+16$ & $90.00^{\circ} \pm 0.82^{\circ}$ & $84.67^{\circ} \pm 3.05^{\circ}$ & $81.00^{\circ} \pm 1.73^{\circ}$ & $57.25^{\circ} \pm 2.50^{\circ}$ \\
\hline $4.55 \mathrm{E}+16$ & $28.40^{\circ} \pm 6.27^{\circ}$ & $44.62^{\circ} \pm 5.56^{\circ}$ & $71.50^{\circ} \pm 2.26^{\circ}$ & $53.37^{\circ} \pm 3.68^{\circ}$ \\
\hline $4.60 \mathrm{E}+16$ & $78.17^{\circ} \pm 6.53^{\circ}$ & $42.86^{\circ} \pm 6.82^{\circ}$ & $81.00^{\circ} \pm 1.82^{\circ}$ & $70.25^{\circ} \pm 1.26^{\circ}$ \\
\hline $6.69 \mathrm{E}+16$ & $91.92^{\circ} \pm 2.06^{\circ}$ & $32.00^{\circ} \pm 6.24^{\circ}$ & $102.80^{\circ} \pm 1.92^{\circ}$ & $44.31^{\circ} \pm 6.29^{\circ}$ \\
\hline
\end{tabular}

\section{Contact angle in each function unit [2 $\mu \mathrm{L} / \mathrm{g}]$}

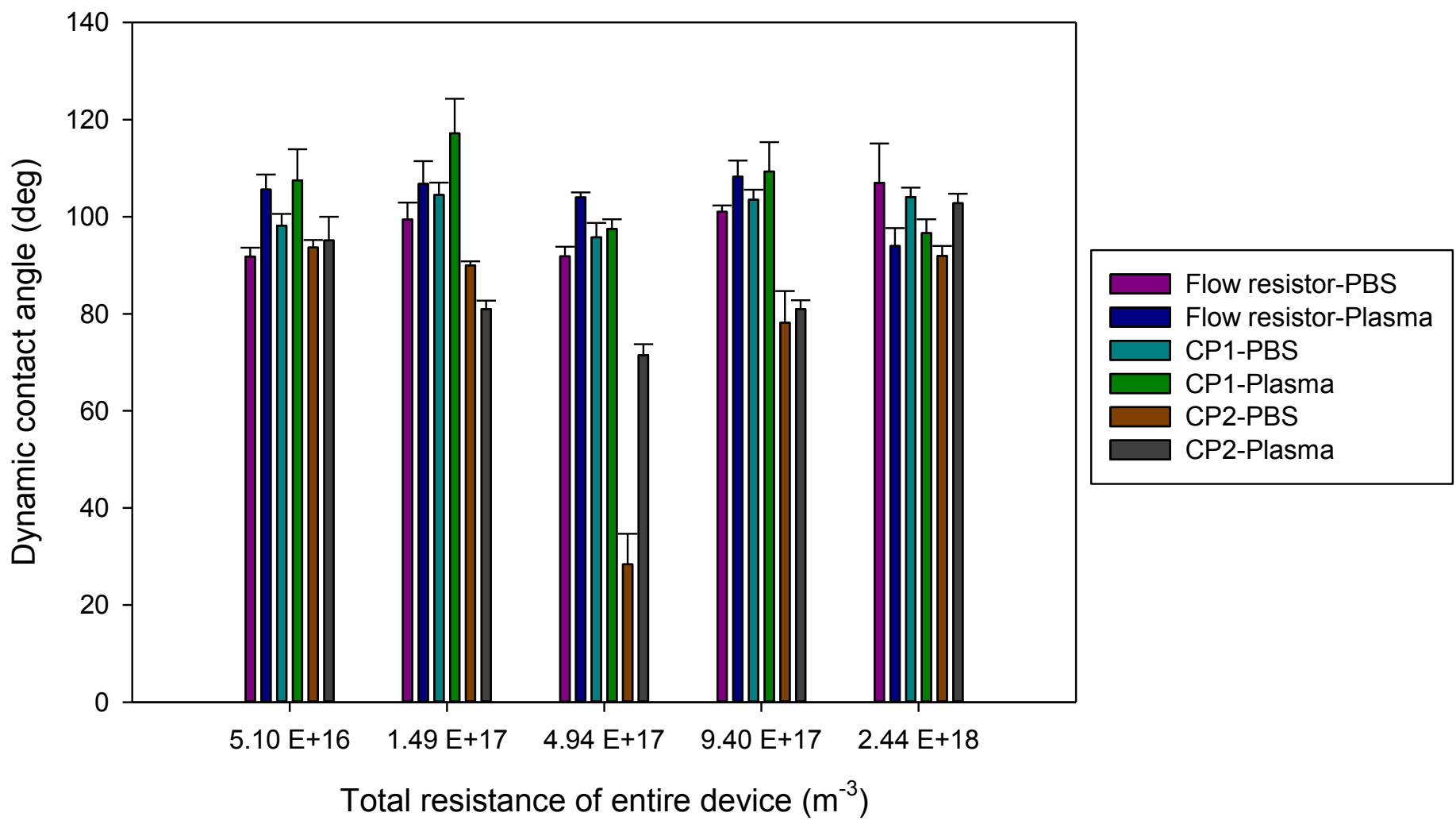

Figure 5-6 Dynamic contact angle in each functional unit of the device using a Pluronic/PDMS concentration of $[2 \mu \mathrm{L} / \mathrm{g}]$ 


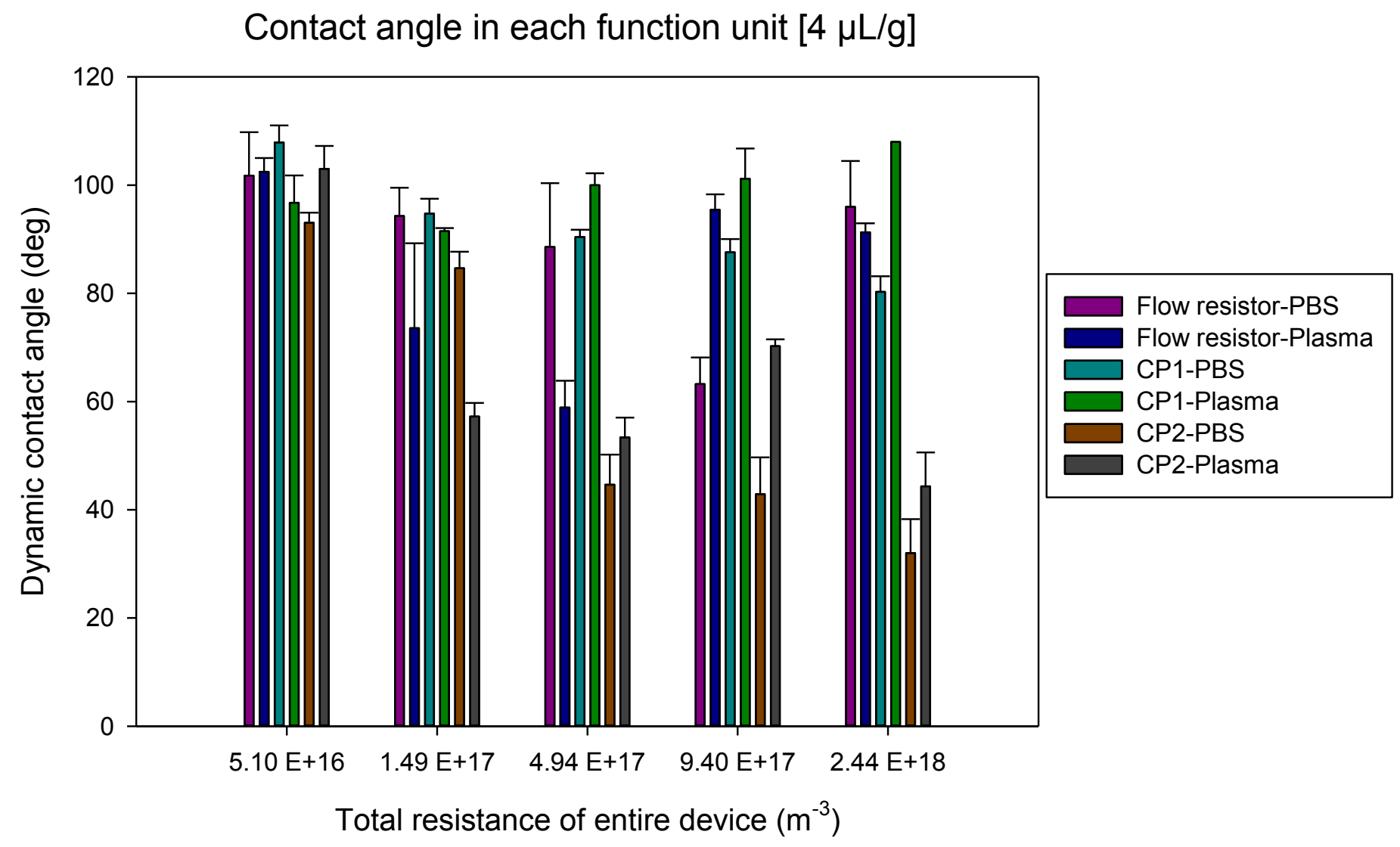

Figure 5-7 Dynamic contact angle in each functional unit of the device using a Pluronic/PDMS concentration of $[4 \mu \mathrm{L} / \mathrm{g}]$

Figures 5-6 and 5-7 show the variation of the dynamic contact angle for each functional unit of the LFD, considering the change in resistance and in the concentration of pluronic. The dynamic contact angle is often lower for higher concentration of pluronic. In addition, the angle usually lowest in magnitude for the secondary capillary pump, as the wetting velocity is decreased as consequence of the additive resistance from all previous functional units (as these units are connected in series).

\subsubsection{Flow rate}

Flow rate was regulated by varying the dimensions of the microstructures in the capillary pump and the spacing between them, as well as the dimensions of the flow resistor, resulting in 
their resistance changes; this allowed for providing more time for the binding of proteins to antibodies, and detection. The capillary pumps determine the filling front of the system, and their resistance is highest when they are almost full; however, the resistance of capillary pumps is few orders of magnitude smaller than the total flow resistance of the capillary system. In addition, the concentration of Pluronic also had to be optimized. In order to be able to predict the effect of the combination of the resistance and the Pluronic concentration on the flow speed, in-situ dynamic contact angle measurements were performed for different solutions, which were then compared with the time-lapse results. The concentration of Pluronic F127 and geometric flow resistances had great impact in the flow speed of the sample. Higher concentrations of Pluronic reduced the contact angle, therefore increasing the hydrophilicity of the device and allowing for higher flow rates. Longer flow resistors encoded a higher fluidic resistance therefore reducing the magnitude of the flow rate. Flow rate was measured by measuring the time required for the fluid to flow through each component of the CS. Table 5-8 shows the relationship between the flow rate of the flow resistor, the geometry based fluidic resistance, and the concentration of Pluronic. The highest flow rate $(18.4 \mathrm{~nL} / \mathrm{s})$ achieved was for a device with no flow resistor (straight channel), and a pluronic concentration of $4 \mu \mathrm{L} / \mathrm{g}$; the lowest flow rate was achieved with a $37 \mathrm{~cm}$ long flow resistor $(0.43 \mathrm{~nL} / \mathrm{s})$, and a concentration of $2 \mu \mathrm{L} / \mathrm{g}$. Flow rate is higher for PBS than Plasma due to its viscosity and surface tension.

Time lapses using the Ti microscope were used to analyze how the flow rate decays over the length of the channel. Table 5-9 shows the flow rate of Plasma at the entrance of the flow resistor, and at the middle of the same for two devices. Even though the flow rate decays over the length of the resistor, the flow rate of the device with lower fluidic resistance is higher at all times. The decay in speed occurs not only due to viscous dissipation forces, but predominately 
due to the increase in length of the filled length in the channel and the friction due to the bulk viscosity [4].

Table 5-8 Flow rate of entire flow resistor

\begin{tabular}{|c|c|c|c|c|}
\hline & \multicolumn{4}{|c|}{$\begin{array}{c}\text { Flow rate of the entire flow resistor } \\
\text { mean } \pm \text { SD }(\mathrm{nL} / \mathrm{s})\end{array}$} \\
\hline $\begin{array}{c}\text { Resistance of the } \\
\text { Flow resistor }\left(\mathrm{m}^{-3}\right)\end{array}$ & $\begin{array}{l}\text { Pluronic/ } \\
\text { PDMS } \\
{[2 \mu \mathrm{L} / \mathrm{g}]}\end{array}$ & $\begin{array}{l}\text { Pluronic/ } \\
\text { PDMS } \\
{[4 \mu \mathrm{L} / \mathrm{g}]}\end{array}$ \\
\hline & PBS & Plasma & PBS & Plasma \\
\hline No flow resistor & $9.20 \pm 0$ & $18.4 \pm 0$ & $18.40 \pm 0$ & $18.40 \pm 0$ \\
\hline $1.02 \mathrm{E}+17$ & $12.15 \pm 0.64$ & $9.94 \pm 0.43$ & $2.06 \pm 1.46$ & $12.60 \pm 0$ \\
\hline $4.35 \mathrm{E}+17$ & $4.08 \pm 0.60$ & $3.17 \pm 0.46$ & $3.12 \pm 0.90$ & $2.37 \pm 0.18$ \\
\hline $8.80 \mathrm{E}+17$ & $2.37 \pm 0.20$ & $1.24 \pm 0.61$ & $1.70 \pm 0.72$ & $1.65 \pm 0.11$ \\
\hline $2.35 \mathrm{E}+18$ & $1.20 \pm 0.07$ & $0.43 \pm 0.10$ & $0.98 \pm 0.06$ & $0.52 \pm 0.01$ \\
\hline
\end{tabular}

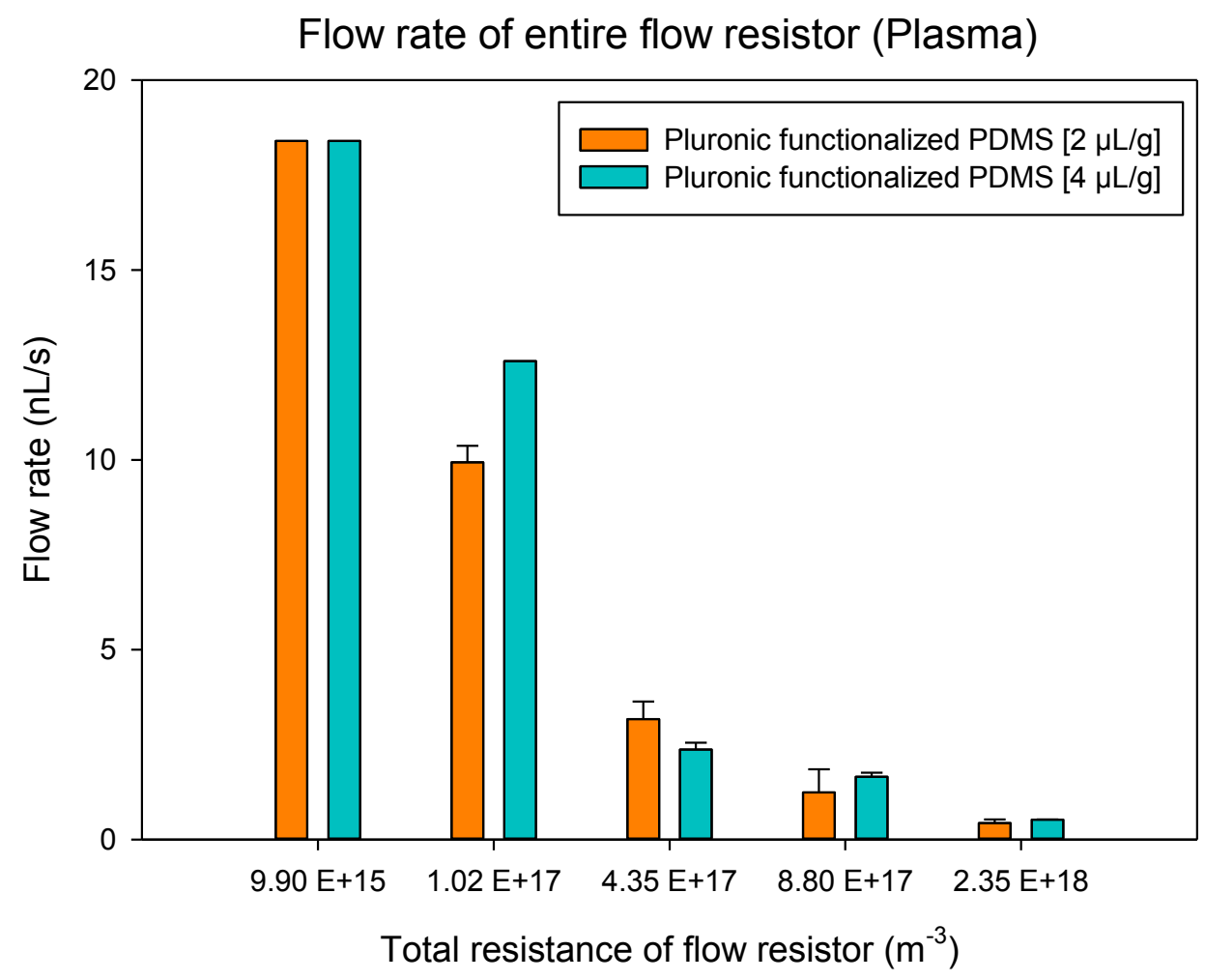

Figure 5-8 Relationship between the geometric resistance variation and the flow rate of plasma. 
Note from Figure 5-8 the general tendency of flow rate to increase as the concentration of pluronic increases, as well as when the geometric resistance is reduced (which occurs at shorter lengths).

Zimmerman et al. had previously reported studies on flow rate control taking advantage of the effect of the secondary capillary pump alone [149]. Their studies were only able to tune the flow in a very short range of velocities $(0.2-3.7 \mathrm{~nL} / \mathrm{s})$ by modifying the dimensions and positioning of the micropillars in the capillary pumps [149]. Even though our work used a set of dimensions for each capillary pump, we took advantage of other factors, including the flow resistor resistance and the concentration of pluronic, and were able to increase this range from 0.43 to $18.4 \mathrm{~nL} / \mathrm{s}$. As previously mentioned, time is a key variable for performing immunoassays using microfluidic technology, so widening the range of flow rates provides much flexibility, and the perfect setting for tuning the time for immunoassays in the future.

Table 5-9 Decay of flow rate across the flow resistor (Pluronic/PDMS $[4 \mu \mathrm{L} / \mathrm{g}]$ )

\begin{tabular}{|l|l|l|l|}
\hline Fluid & $\begin{array}{l}\text { Flow resistance } \\
\left(\mathrm{m}^{-3}\right)\end{array}$ & $\begin{array}{l}\text { Flow rate } \\
\text { entrance } \\
\text { mean } \pm \mathrm{SD} \\
(\mathrm{nL} / \mathrm{s})\end{array}$ & $\begin{array}{l}\text { Flow rate } \\
\text { middle } \\
\text { mean } \pm \mathrm{SD} \\
(\mathrm{nL} / \mathrm{s})\end{array}$ \\
\hline Plasma & $4.35 \mathrm{E}+17$ & $2.99 \pm 0.14$ & $1.31 \pm 0.07$ \\
\cline { 2 - 4 } & $8.80 \mathrm{E}+17$ & $1.93 \pm 0.32$ & $0.71 \pm 0.06$ \\
\hline
\end{tabular}

Tables 5-10 and 5-11 show the relationship between the flow rate of the Capillary pumps, the geometry based fluidic resistance, and the concentration of Pluronic. Flow rate was highly consistent for $\mathrm{CP} 1$ due to the fact that a large portion consists of an open window where the plasma separation membrane is placed (Figure 5-9). The flow rate in CP2 had a general tendency to increase as resistance decreased and as concentration of pluronic increased (Figure 5-10). 
Table 5-10 Flow rate in first capillary pump

\begin{tabular}{|c|c|c|c|c|}
\hline \multirow{3}{*}{$\begin{array}{l}\text { Resistance of the } \\
\text { Capillary pump } \\
\left(\mathrm{m}^{-3}\right)\end{array}$} & \multicolumn{4}{|c|}{$\begin{array}{l}\text { Flow rate of the first capillary pump } \\
\text { mean } \pm \mathrm{SD}(\mathrm{nL} / \mathrm{s})\end{array}$} \\
\hline & \multicolumn{2}{|l|}{$\begin{array}{l}\text { Pluronic/ } \\
\text { PDMS } \\
{[2 \mu \mathrm{L} / \mathrm{g}]}\end{array}$} & \multicolumn{2}{|l|}{$\begin{array}{l}\text { Pluronic/ } \\
\text { PDMS } \\
{[4 \mu \mathrm{L} / \mathrm{g}]}\end{array}$} \\
\hline & PBS & Plasma & PBS & Plasma \\
\hline $7.73 \mathrm{E}+15$ & $818.40 \pm 0$ & $818.40 \pm 0$ & $818.40 \pm 0$ & 818.40 \\
\hline $8.69 \mathrm{E}+15$ & $786.70 \pm 0$ & $786.70 \pm 0$ & $786.70 \pm 0$ & $786.70 \pm 0$ \\
\hline $1.11 \mathrm{E}+16$ & $723.30 \pm 0$ & $723.30 \pm 0$ & $723.30 \pm 0$ & 723.30 \\
\hline $1.13 \mathrm{E}+16$ & 720.40 & $720.40 \pm 0$ & 720.40 & $720.40 \pm 0$ \\
\hline $1.64 \mathrm{E}+16$ & $633.97 \pm 0$ & $633.97 \pm 0$ & $633.97 \pm 0$ & $633.97 \pm 0$ \\
\hline
\end{tabular}

Flow rate of first capillary pump

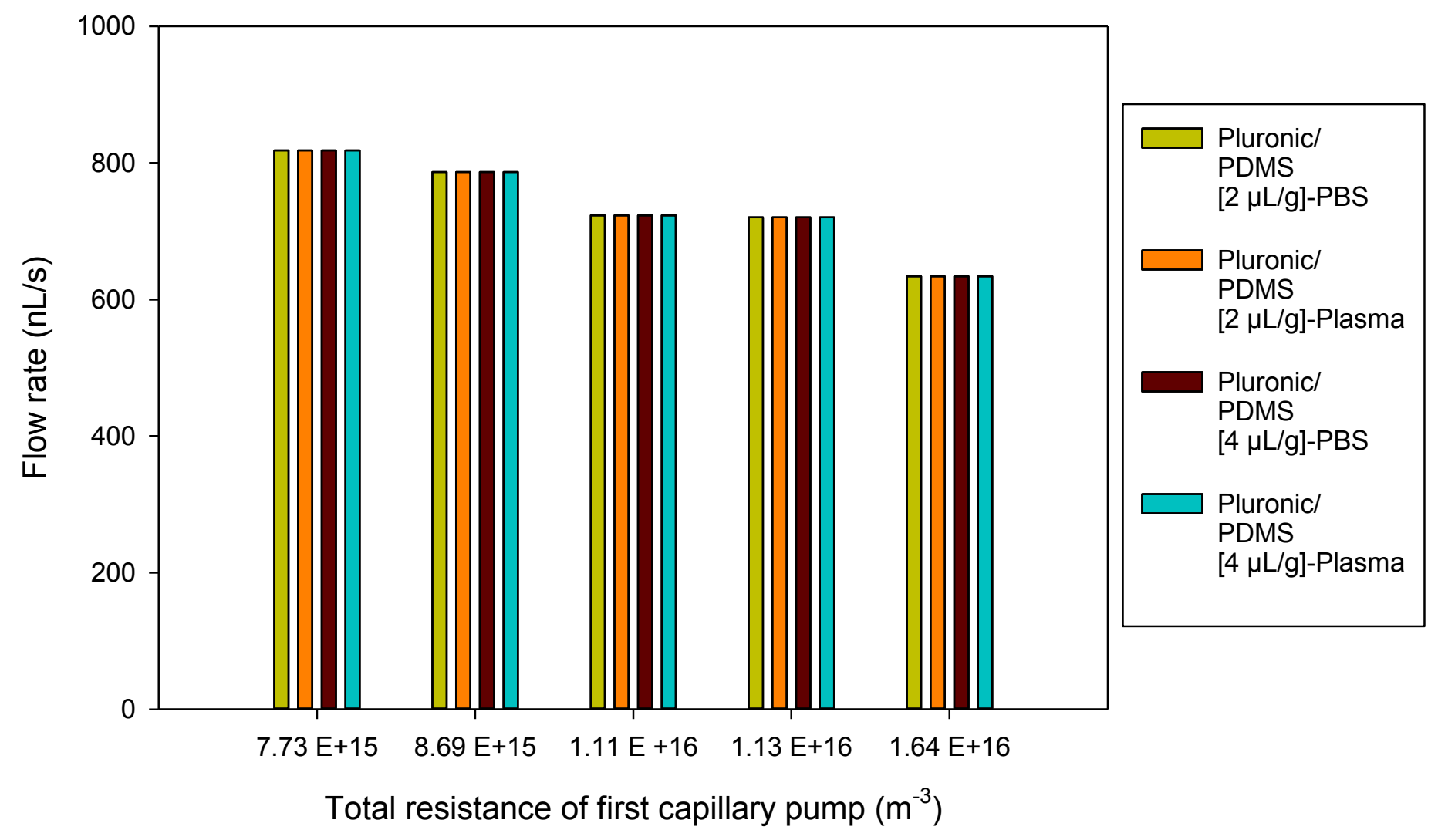

Figure 5-9 Flow rate in first capillary pump 
Table 5-11 Flow rate in second capillary pump

\begin{tabular}{|c|c|c|c|c|}
\hline \multirow{3}{*}{$\begin{array}{l}\text { Resistance of the } \\
\text { Capillary pump } \\
\left(\mathrm{m}^{-3}\right)\end{array}$} & \multicolumn{4}{|c|}{$\begin{array}{l}\text { Flow rate of the second capillary pump } \\
\text { mean } \pm \mathrm{SD}(\mathrm{nL} / \mathrm{s})\end{array}$} \\
\hline & \multicolumn{2}{|l|}{$\begin{array}{l}\text { Pluronic/ } \\
\text { PDMS } \\
{[2 \mu \mathrm{L} / \mathrm{g}]}\end{array}$} & \multicolumn{2}{|l|}{$\begin{array}{l}\text { Pluronic/ } \\
\text { PDMS } \\
{[4 \mu \mathrm{L} / \mathrm{g}]}\end{array}$} \\
\hline & PBS & Plasma & PBS & Plasma \\
\hline $3.17 \mathrm{E}+16$ & 30.62 & $38.55 \pm 7.85$ & $45.49 \pm 15.44$ & $34.61 \pm 1.58$ \\
\hline $3.56 \mathrm{E}+16$ & $15.19 \pm 1.83$ & $8.88 \pm 1.80$ & $15.36 \pm 0.32$ & $12.11 \pm 3.72$ \\
\hline $4.55 \mathrm{E}+16$ & $4.22 \pm 0.65$ & $2.08 \pm 0.52$ & $3.03 \pm 0.69$ & $1.43 \pm 0.46$ \\
\hline $4.60 \mathrm{E}+16$ & $1.63 \pm 0.29$ & $0.90 \pm 0$ & $1.88 \pm 0.56$ & $1.34 \pm 0.06$ \\
\hline $6.69 \mathrm{E}+16$ & $0.94 \pm 0.09$ & 0.32 & $1.00 \pm 0.14$ & $0.61 \pm 0.11$ \\
\hline
\end{tabular}

\section{Flow rate of second capillary pump}

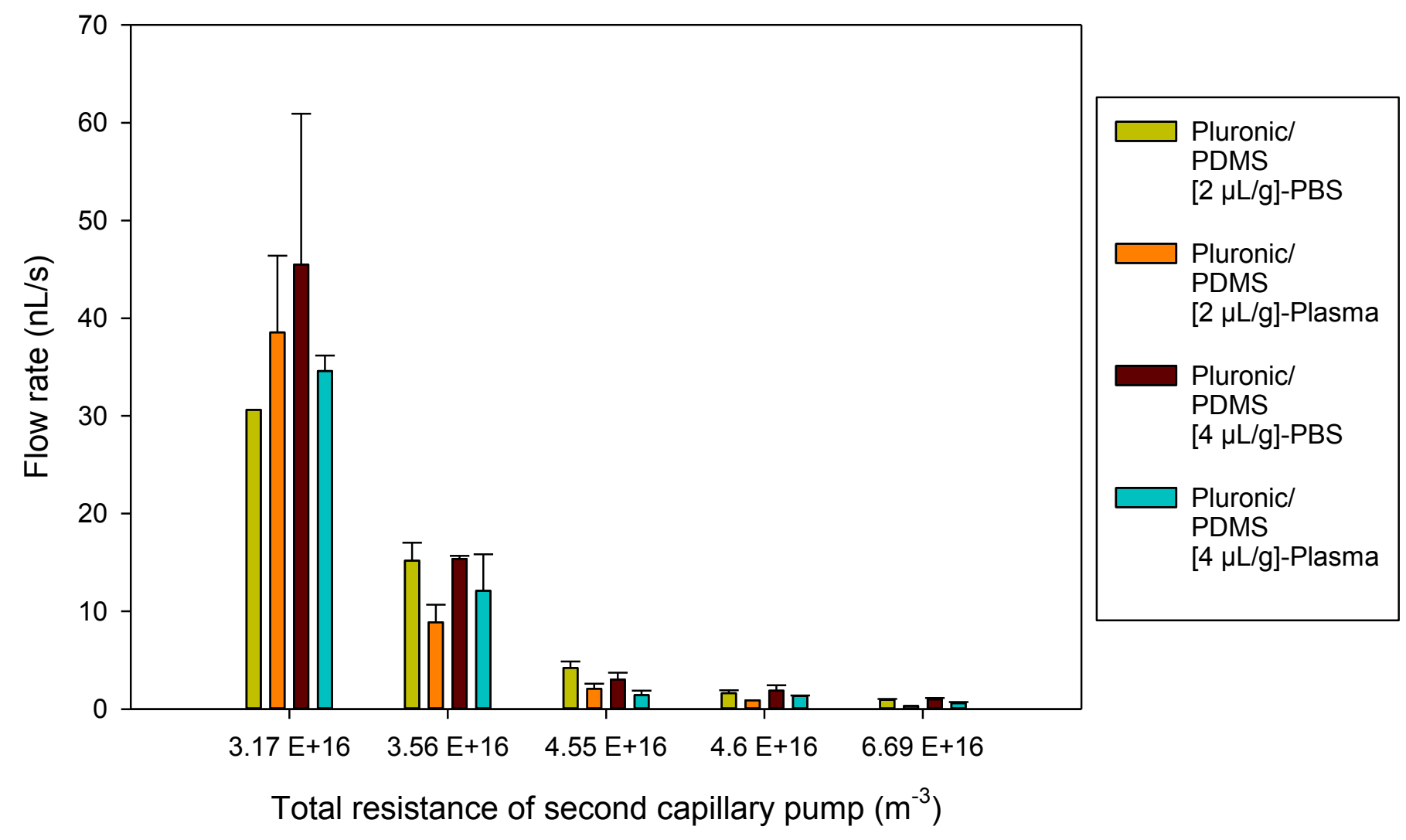

Figure 5-10 Flow rate of secondary capillary pump

\subsubsection{Water Treatment}


As previously mentioned, the properties of the Pluronic in the PDMS where further enhanced by DI water treating the LFD in order to trigger the gradient-induced migration of the pluronic embedded in the PDMS. This treatment would further enhance the hydrophilic properties of the microfluidic channels, and it would also make the surface more stable and less prompt to nonspecific adsorption. Two methods where compared. The first consisted of fully immersing the LFD in DI water for 24 hours, and the second consisted in placing the patterned surface of the fluidic layer as well as the surface of the interfacing layer in contact with the DI water for the same 24 hours (Figure 5-11). Contact angles were measured using both methods, and proved to be much lower for the devices partially immersed in water compared to those fully immersed (Tables 5-16 and 5-17); flow rate was in consequence higher for the partially immersed devices.

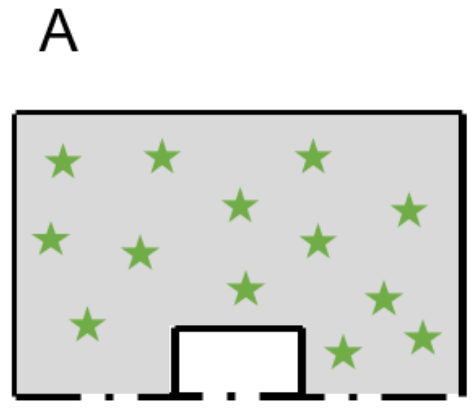

PDMS-Pluronic
B

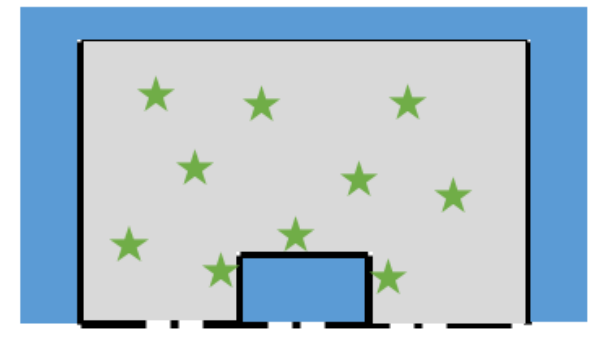

PDMS
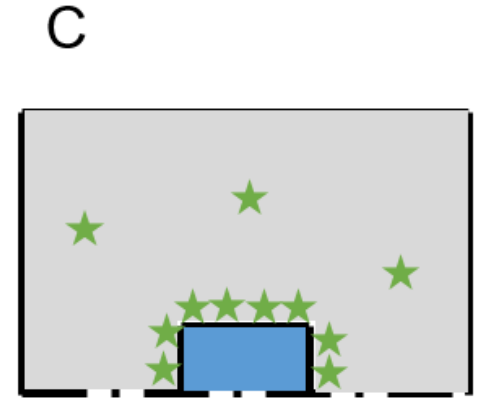

Air

Figure 5-11 Schematic of process of surface modification. A) Before water treatment. B) Water treatment by full immersion. C) Water treatment by partial immersion.

Flow rate was also measured and compared for devices with and without the water treatment, for both the entire device and the flow resistor portion (Tables 5-12 to 5-14). Even though contact angles proved to be more hydrophilic (Figure 5-12), and flow rate was higher at the entrance of the channels, it was observed during experimentation that the effects of 
evaporation where more evident in water treated devices, which resulted in the full length flow rate been lower compared to that of the non-treated ones.

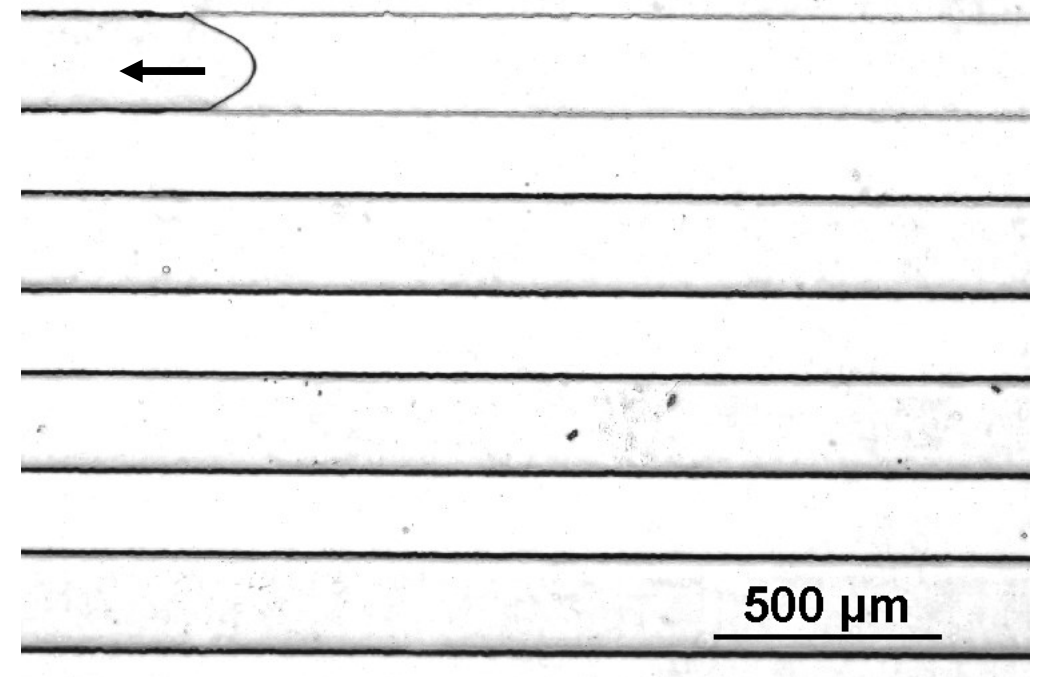

Figure 5-12 Contact angle of water treated (partially immersed) LFD at the flow resistor. The concentration of pluronic was $4 \mu \mathrm{L} / \mathrm{g}$. The black arrow indicates the direction of the flow.

Table 5-12 Flow rate of entire device without water treatment

\begin{tabular}{|l|l|l|l|l|l|}
\hline & \multicolumn{5}{|l|}{$\begin{array}{l}\text { Flow rate of the entire device } \\
\text { mean } \pm \mathrm{SD}(\mathrm{nL} / \mathrm{s})\end{array}$} \\
\hline $\begin{array}{l}\text { Resistance of } \\
\text { the Entire } \\
\text { Device }\left(\mathrm{m}^{-3}\right)\end{array}$ & $\begin{array}{l}\text { Resistance of } \\
\text { the Flow } \\
\text { resistor }\left(\mathrm{m}^{-3}\right)\end{array}$ & $\begin{array}{l}\text { Pluronic/ } \\
\text { PDMS } \\
{[2 \mu \mathrm{L} / \mathrm{g}]}\end{array}$ & $\begin{array}{l}\text { Pluronic/ } \\
\text { PDMS } \\
{[4 \mu \mathrm{L} / \mathrm{g}]}\end{array}$ & \\
\hline & & PBS & Plasma & PBS & Plasma \\
\hline $5.10 \mathrm{E}+16$ & $\begin{array}{l}\text { No flow } \\
\text { resistor }\end{array}$ & $101.11 \pm 5.21$ & $60.70 \pm 0.98$ & $127.77 \pm 9.77$ & $61.30 \pm 4.62$ \\
\hline $1.49 \mathrm{E}+17$ & $1.02 \mathrm{E}+17$ & $29.32 \pm 1.18$ & $18.88 \pm 2.27$ & $32.18 \pm 1.10$ & $27.76 \pm 8.16$ \\
\hline $4.94 \mathrm{E}+17$ & $4.35 \mathrm{E}+17$ & $8.83 \pm 1.50$ & $4.96 \pm 1.17$ & $6.35 \pm 1.87$ & $5.23 \pm 1.66$ \\
\hline $9.40 \mathrm{E}+17$ & $8.80 \mathrm{E}+17$ & $3.61 \pm 0.32$ & $2.02 \pm 0.05$ & $3.59 \pm 1.44$ & $2.86 \pm 0.19$ \\
\hline $2.44 \mathrm{E}+18$ & $2.35 \mathrm{E}+18$ & $1.87 \pm 0.16$ & $2.32 \pm 0.22$ & $1.71 \pm 0.06$ & $0.99 \pm 0.09$ \\
\hline
\end{tabular}


Table 5-13 Flow rate of entire device with water treatment

\begin{tabular}{|l|l|l|l|}
\hline & & \multicolumn{2}{|l|}{$\begin{array}{l}\text { Flow rate of the entire device } \\
\text { mean } \pm \mathrm{SD}(\mathrm{nL} / \mathrm{s})\end{array}$} \\
\hline $\begin{array}{l}\text { Resistance of } \\
\text { the Entire } \\
\text { Device }\left(\mathrm{m}^{-3}\right)\end{array}$ & $\begin{array}{l}\text { Resistance of } \\
\text { the Flow } \\
\text { resistor }\left(\mathrm{m}^{-3}\right)\end{array}$ & $\begin{array}{l}\text { Pluronic/ } \\
\text { PDMS } \\
{[2 \mu \mathrm{L} / \mathrm{g}]}\end{array}$ & $\begin{array}{l}\text { Pluronic/ } \\
\text { PDMS } \\
{[4 \mu \mathrm{L} / \mathrm{g}]}\end{array}$ \\
\hline & & Plasma & Plasma \\
\hline $5.10 \mathrm{E}+16$ & $\begin{array}{l}\text { No flow } \\
\text { resistor }\end{array}$ & $56.72 \pm 6.35$ & $31.79 \pm 5.69$ \\
\hline $1.49 \mathrm{E}+17$ & $1.02 \mathrm{E}+17$ & $23.49 \pm 1.04$ & $28.57 \pm 4.10$ \\
\hline $4.94 \mathrm{E}+17$ & $4.35 \mathrm{E}+17$ & $3.84 \pm 1.27$ & $3.13 \pm 0.24$ \\
\hline $9.40 \mathrm{E}+17$ & $8.80 \mathrm{E}+17$ & $1.96 \pm 0.12$ & $1.85 \pm 0.84$ \\
\hline $2.44 \mathrm{E}+18$ & $2.35 \mathrm{E}+18$ & $1.48 \pm 0.09$ & $1.62 \pm 0.38$ \\
\hline
\end{tabular}

Figure 5-13 shows the effect of the resistance, the concentration of pluronic and the water treatment on the flow rate of the entire device. The resistance has the most impact on the flow rate.

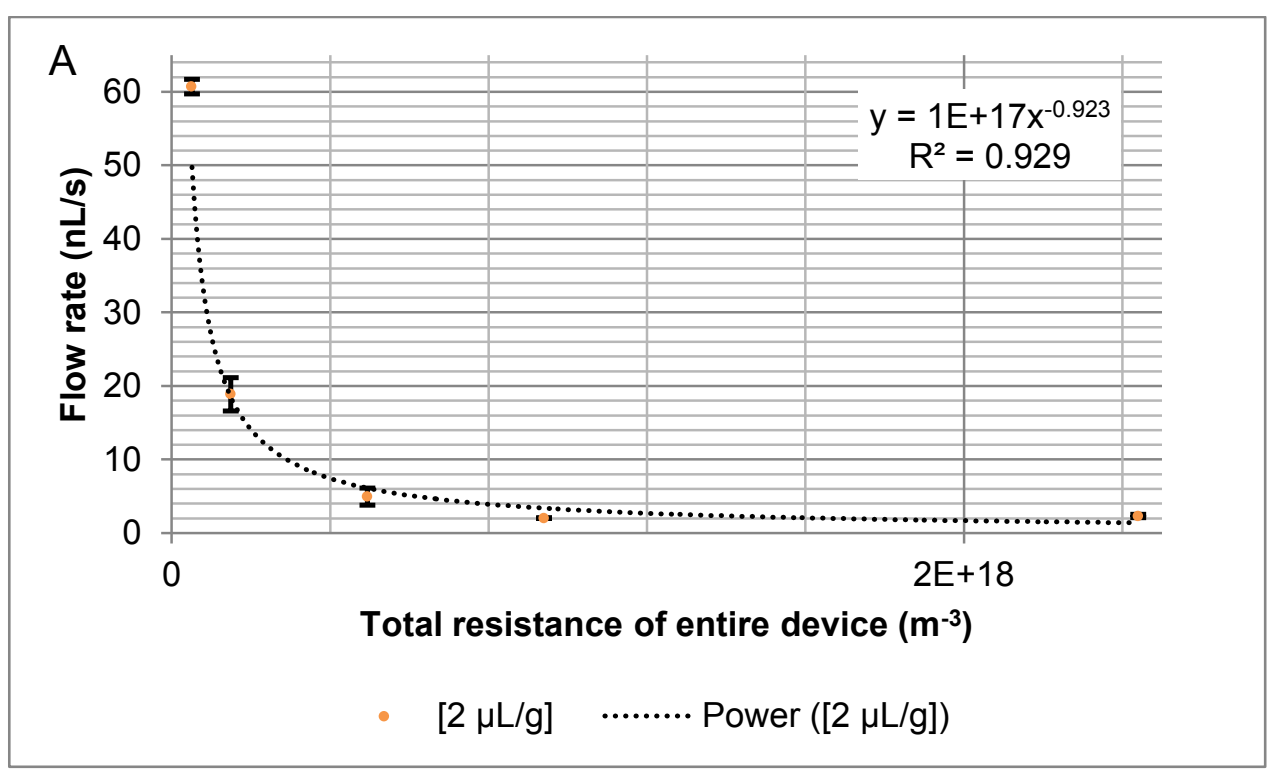



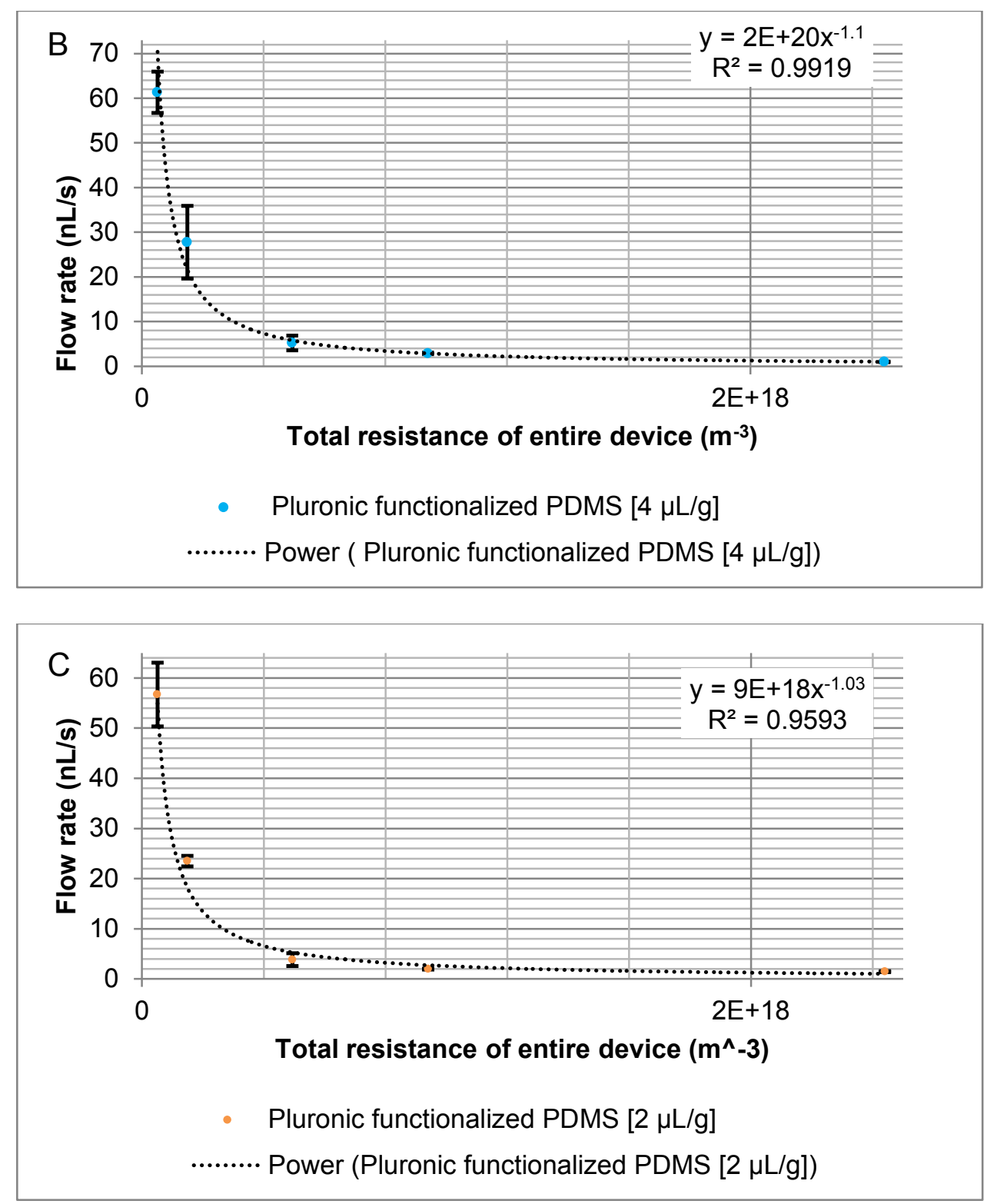


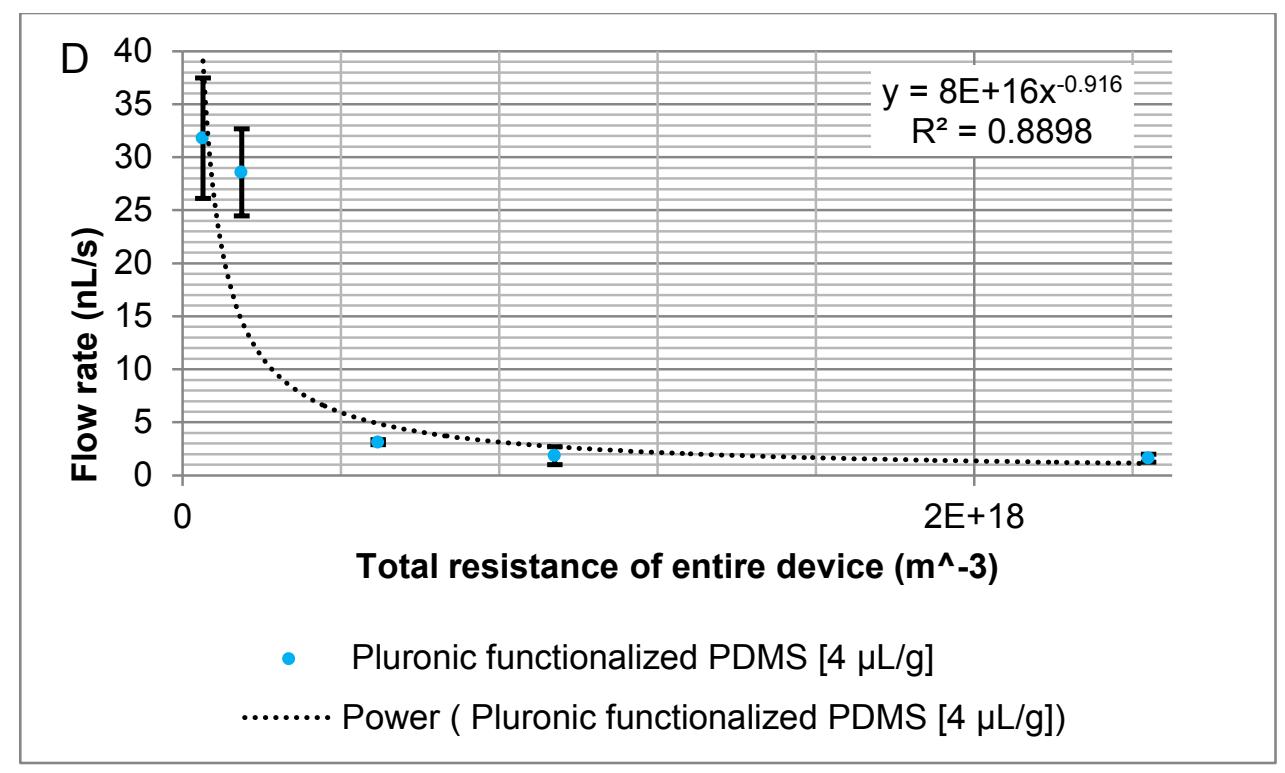

Figure 5-13 Flow rate of the entire device. A) Pluronic Functionalized PDMS [2 $\mu \mathrm{L} / \mathrm{g}]$ without water treartment. B) Pluronic Functionalized PDMS [4 $\mu \mathrm{L} / \mathrm{g}$ ] without water treartment. C) Pluronic Functionalized PDMS [2 $\mu \mathrm{L} / \mathrm{g}]$ with water treartment. D) Pluronic Functionalized PDMS $[4 \mu \mathrm{L} / \mathrm{g}]$ with water treartment.

Table 5-14 Flow rate of entire flow resistor with water treatment

\begin{tabular}{|l|l|l|}
\hline & \multicolumn{2}{|l|}{$\begin{array}{l}\text { Flow rate of the entire flow resistor } \\
\text { mean } \pm \text { SD }(\mathrm{nL} / \mathrm{s})\end{array}$} \\
\hline $\begin{array}{l}\text { Resistance of } \\
\text { the Flow } \\
\text { resistor }\left(\mathrm{m}^{-3}\right)\end{array}$ & $\begin{array}{l}\text { Pluronic/ } \\
\text { PDMS } \\
{[2 \mu \mathrm{L} / \mathrm{g}]}\end{array}$ & $\begin{array}{l}\text { Pluronic/ } \\
\text { PDMS } \\
{[4 \mu \mathrm{L} / \mathrm{g}]}\end{array}$ \\
\hline & Plasma & Plasma \\
\hline $\begin{array}{l}\text { No flow } \\
\text { resistor }\end{array}$ & $2.96 \pm 0.22$ & $3.27 \pm 1.37$ \\
\hline $1.02 \mathrm{E}+17$ & $2.82 \pm 0.72$ & $3.61 \pm 0.28$ \\
\hline $4.35 \mathrm{E}+17$ & $3.55 \pm 1.00$ & $1.99 \pm 0.45$ \\
\hline $8.80 \mathrm{E}+17$ & $1.04 \pm 0.22$ & $1.72 \pm 0.04$ \\
\hline $2.35 \mathrm{E}+18$ & $0.70 \pm 0.08$ & $0.86 \pm 0.25$ \\
\hline
\end{tabular}

Both static and dynamic contact angles where measured to observe the effect of the water treatment (Table 5-15 to 5-17). 
Table 5-15 Static contact angle after water treatment

\begin{tabular}{|l|l|l|}
\hline & Plasma \\
\hline & Pluronic/PDMS [2 $\mu \mathrm{L} / \mathrm{g}]$ & Pluronic/PDMS $[4 \mu \mathrm{L} / \mathrm{g}]$ \\
\hline Static contact angle (mean \pm SD) & $65.60^{\circ} \pm 2.67^{\circ}$ & $69.74^{\circ} \pm 1.65^{\circ}$ \\
\hline
\end{tabular}

\section{Static contact angle of plasma on Pluronic functionalized PDMS}

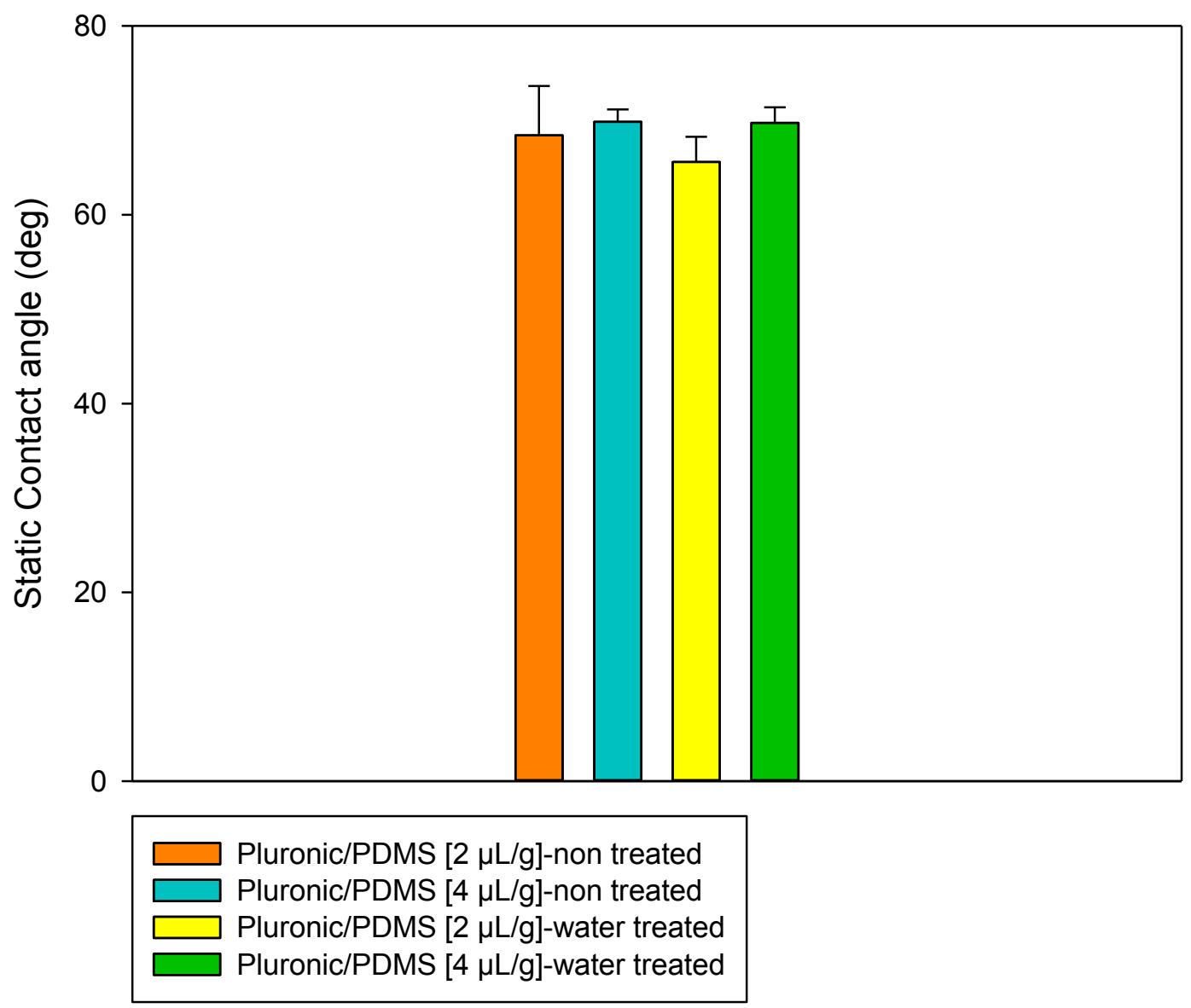

Figure 5-14 Comparison of static contact angle with and without water treatment

The water treated static contact angle only presented a very slight decrease in comparison of its non-treated counterpart (Figure 5-14). However, the dynamic contact angles presented a more evident change; contact angles are generally lower for the partially immersed devices since this treatment triggers a gradient, leading the pluronic molecules to merge to the surface of the microchannels (Figure 5-15). 
Table 5-16 Dynamic contact angle of flow resistor after water treatment (full immersion)

\begin{tabular}{|l|l|l|}
\hline & \multicolumn{2}{|l|}{$\begin{array}{l}\text { Contact angle of flow resistor after } \\
\text { water treatment } \\
\text { mean } \pm \text { SD }(\mathrm{nL} / \mathrm{s})\end{array}$} \\
\hline $\begin{array}{l}\text { Resistance of } \\
\text { the Flow } \\
\text { resistor }\left(\mathrm{m}^{-3}\right)\end{array}$ & $\begin{array}{l}\text { Pluronic/ } \\
\text { PDMS } \\
{[2 \mu \mathrm{L} / \mathrm{g}]}\end{array}$ & $\begin{array}{l}\text { Pluronic/ } \\
\text { PDMS } \\
{[4 \mu \mathrm{L} / \mathrm{g}]}\end{array}$ \\
\hline $\begin{array}{l}\text { No flow } \\
\text { resistor }\end{array}$ & Plasma & Plasma \\
\hline $1.02 \mathrm{E}+17$ & $95.75^{\circ} \pm 3.20^{\circ}$ & $84.50^{\circ} \pm 4.95^{\circ}$ \\
\hline $4.35 \mathrm{E}+17$ & $97.00^{\circ} \pm 3.56^{\circ}$ & $36.92^{\circ} \pm 7.97^{\circ}$ \\
\hline $8.80 \mathrm{E}+17$ & $76.37^{\circ} \pm 0.92^{\circ}$ & $67.75^{\circ} \pm 4.35^{\circ}$ \\
\hline $2.35 \mathrm{E}+18$ & $99.25^{\circ} \pm 8.75^{\circ}$ & $56.96^{\circ} \pm 2.12^{\circ}$ \\
\hline
\end{tabular}

Table 5-17 Dynamic contact of flow resistor after water treatment (patterned face in contact with water)

\begin{tabular}{|l|l|l|}
\hline & \multicolumn{2}{|l|}{$\begin{array}{l}\text { Contact angle of flow resistor after } \\
\text { water treatment } \\
\text { mean } \pm \text { SD }(\mathrm{nL} / \mathrm{s})\end{array}$} \\
\hline $\begin{array}{l}\text { Resistance of } \\
\text { the Flow } \\
\text { resistor }\left(\mathrm{m}^{-3}\right)\end{array}$ & $\begin{array}{l}\text { Pluronic/ } \\
\text { PDMS } \\
{[2 \mu \mathrm{L} / \mathrm{g}]}\end{array}$ & $\begin{array}{l}\text { Pluronic/ } \\
\text { PDMS } \\
{[4 \mu \mathrm{L} / \mathrm{g}]}\end{array}$ \\
\hline & Plasma & Plasma \\
\hline $\begin{array}{l}\text { No flow } \\
\text { resistor }\end{array}$ & $76.5^{\circ} \pm 9.25^{\circ}$ & $95.83^{\circ} \pm 4.95^{\circ}$ \\
\hline $1.02 \mathrm{E}+17$ & $57.25^{\circ} \pm 4.86^{\circ}$ & $68.21^{\circ} \pm 3.87^{\circ}$ \\
\hline $4.35 \mathrm{E}+17$ & $54.89^{\circ} \pm 5.92^{\circ}$ & $67.00^{\circ} \pm 9.53^{\circ}$ \\
\hline $8.80 \mathrm{E}+17$ & $45.46^{\circ} \pm 3.60^{\circ}$ & $45.46^{\circ} \pm 3.60^{\circ}$ \\
\hline $2.35 \mathrm{E}+18$ & $38.00^{\circ} \pm 6.68^{\circ}$ & $44.00^{\circ} \pm 9.11^{\circ}$ \\
\hline
\end{tabular}




\section{Dynamic contact angle in flow resistor}

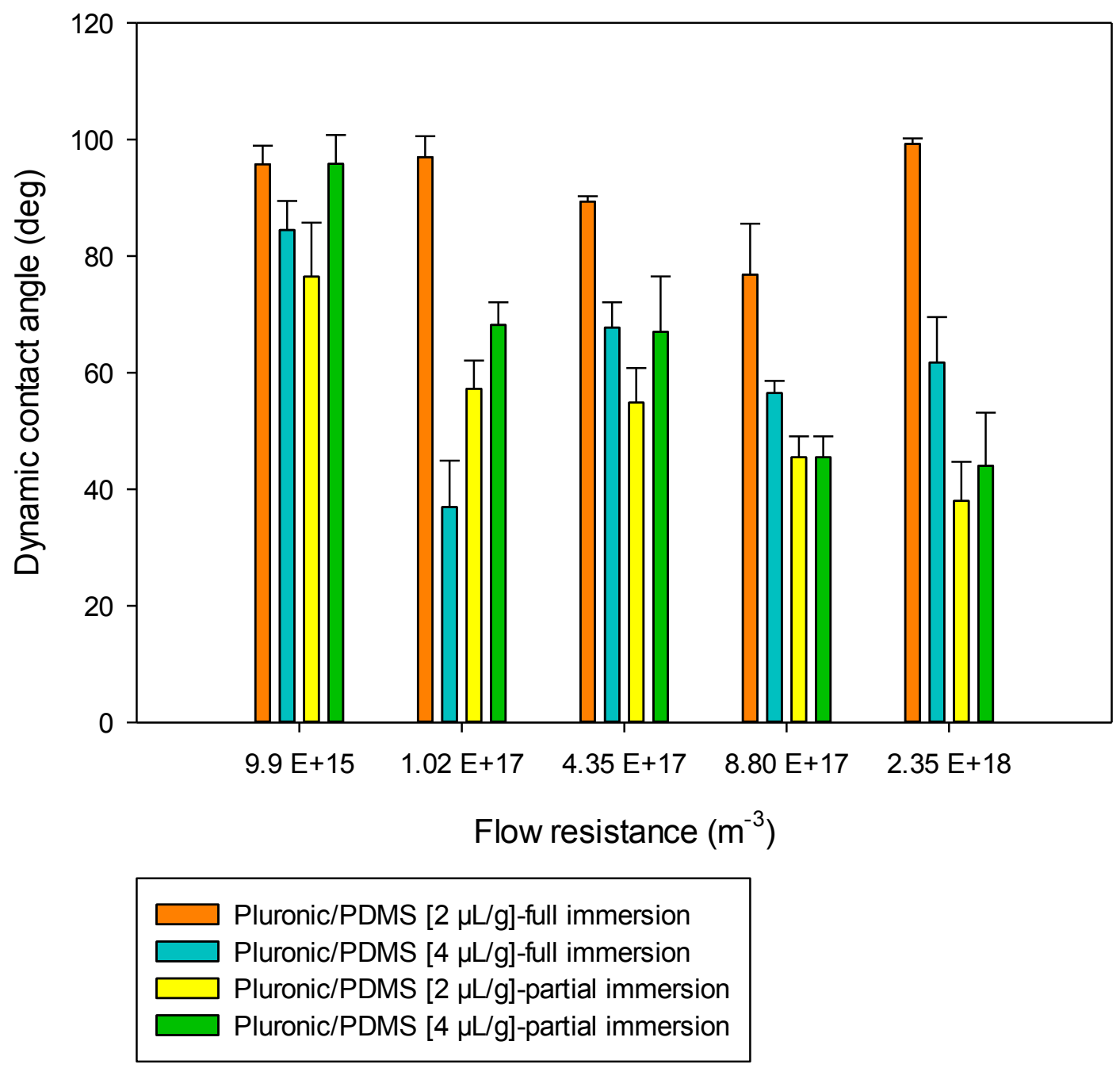

Figure 5-15 Comparison of dynamic contact angle of water treated devices based on the immersion mode.

Finally, the experimental results were validated using the equations in chapter 3 . Both the static and dynamic contact angles were evaluated separately to determine which one would be a better predictor of the flow rate. Results showed that the static contact angles could provide a good approximate to the experimental results, while the dynamic contact angles were a little more complex to use in the flow equations since the contact with two of the microchannel walls remains unknown. 
Table 5-18 Validation data (Plasma)

\begin{tabular}{|c|c|c|}
\hline & \multicolumn{2}{|c|}{ Flow rate of the entire flow resistor mean \pm SD $(\mathrm{nL} / \mathrm{s})$} \\
\hline Resistance of the Flow resistor $\left(\mathrm{m}^{-3}\right)$ & Pluronic/PDMS $[2 \mu \mathrm{L} / \mathrm{g}]$ & Pluronic/PDMS $[4 \mu \mathrm{L} / \mathrm{g}]$ \\
\hline $1.02 \mathrm{E}+17$ & 9.31 & 8.72 \\
\hline $4.35 \mathrm{E}+17$ & 2.35 & 2.20 \\
\hline $8.80 \mathrm{E}+17$ & 1.17 & 0.46 \\
\hline $2.35 \mathrm{E}+18$ & 0.49 & 1.09 \\
\hline
\end{tabular}

Comparison of theoretical and experimental flow rate

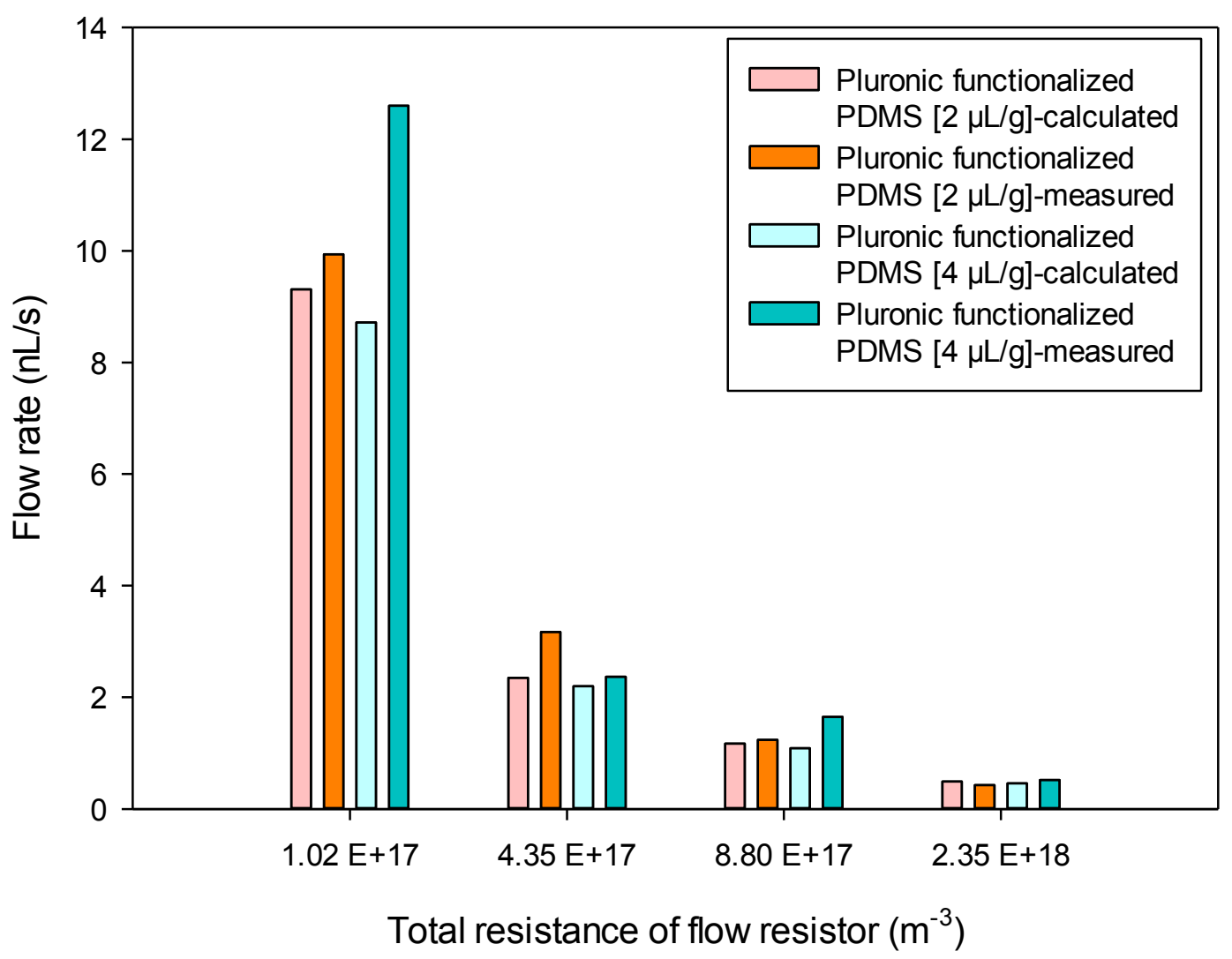

Figure 5-16 Comparison of the experimentally measured and the theoretically calculated flow rate using the static contact angles.

Table 5-18 shows the calculated flow rate using the equations in chapter 3 , and assuming the four angles to be equivalent to the measured static contact angle. Results showed that static 
contact angle can serve as good predictor of the flow rate, even though it does not account for the resistance of the microchannels (Figure 5-16).

\subsection{Evaluation of the Integrated LFD with Human Whole Blood}

\subsubsection{Background}

A major requirement for the development of point-of-care tests for the detection of disease analytes is the need to separate high quality plasma from whole blood efficiently and rapidly [151]. Methods such as centrifugation require additional equipment to separate the plasma fraction from the red blood cells [151], so they are not convenient for point of care applications. The highly asymmetric nature of a plasma separation membrane allows the cellular components of the blood (red cells, white cells, and platelets) to be captured in the larger pores without lysis, while the plasma flows down into the smaller pores on the downstream side of the membrane [152]. Additionally, plasma separation membranes are inexpensive and easy to integrate in microfluidic platforms.

\subsubsection{Method}

The blood separation membrane Vivid ${ }^{\mathrm{TM}}$ GR (Pall Corporation) was selected for the utilization in the LFD because it has the highest plasma yield $(\sim 80 \%)$ according to the manufacturer. After the LFD was assembled, the plasma separation membrane was cut of a slightly smaller size than that of the window opening $(18 \mathrm{~mm}$ by $5 \mathrm{~mm})$, and placed on top of the CP1 (dull size facing up). The contact between the membrane and the CP structures needs to be

very good for a successfully separation (Figure 4-1). The $0.9 \mathrm{~cm}^{2}$ membrane can separate up to $36 \mu \mathrm{L}$ of plasma from $45 \mu \mathrm{L}$ of whole blood. $30 \mu \mathrm{L}$ of whole blood were then deposited on top 
of the membrane and slowly absorbed by it. Red blood cells, white blood cells, and platelets were trapped on top part of the membrane due to their size being larger than the membrane's pores, and only plasma ( $24 \mu \mathrm{L})$ was able to pass through the membrane and then driven through the channel.

\subsection{Microscope Imaging and Data Analysis}

\subsubsection{Microscope Imaging}

A Nikon Eclipse Ti microscope was used to image and evaluate the flow separation capabilities of the membrane for whole blood separation and the flow speeds for fluidic driven (such as plasma and PBS). During the imaging, a $40 \mu \mathrm{L}$ droplet of liquid was placed in the inlet port of the device, and the fluid was transported by capillarity and a time-lapse recording was taken in the flow resistor area in order to observe the combined effect of the flow resistance and the Pluronic concentration. For the analysis of the blood separation efficiency, $30 \mu \mathrm{L}$ of human whole blood (Innovative Research) were deposited on the GR plasma separation membrane, and a time-lapse recording was taken in the $\mathrm{CP} 1$ area to image a contact region of the plasma separation membrane with the $\mathrm{CP} 1$ microstructures.

\subsubsection{Data Analysis}

Flow speed was analyzed at the entrance and middle of the flow resistor to observe its dynamic change due to the pressure drop between the beginning and end of the rectangular microchannel [153]. The overall flow rate of the device was calculated by timing the fluid from entrance to exit of each component, and then dividing the volume of the specific component by the time the fluid spent going across it. Blood separation efficiency was analyzed using microscopy techniques to evaluate the quality of the plasma separated. Flow rate and flow speed 
analysis were performed using the time-lapse results by the NIS-Elements Viewer (Nikon Instruments Inc.). Dynamic contact angle measurements were performed manually using a protractor. For statistical analysis, data was presented as the mean \pm standard deviation (SD) and each individual experiment was performed at least three times $(n \geq 3)$.

\subsubsection{Results}

High quality separated plasma (no leakage of red or white blood cells) was obtained with the GR membrane. Figure 5-17 shows the plasma separation process, beginning by pipetting human whole blood is pipetted on top of the GR membrane.

Table 5-19 compares the average velocity of the fluid at the entrance of the flow resistor for commercially available plasma (separated using other methods such as centrifugation) and plasma separated using the Vivid GR separation membrane. The velocity of the separated plasma was highly consistent with that of the centrifuged plasma.
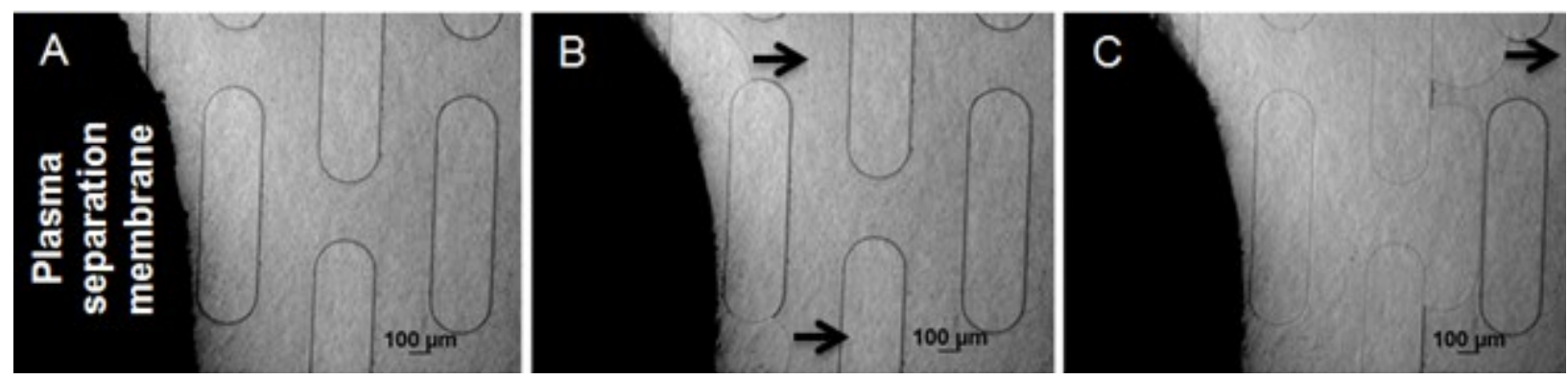

Figure 5-17 Plasma separation from whole blood. A) Ready to separate. B) Start to separate. C) Continuous separation. The black arrows indicate the direction of the flow. 
Table 5-19 Comparison of flow rates of the flow resistor (entrance) for separated plasma from whole blood and just plasma. The substrate used was Pluronic functionalized PDMS (Pluronic/PDMS $[4 \mu \mathrm{L} / \mathrm{g}]$ ).

\begin{tabular}{|l|l|l|r|}
\hline \multicolumn{2}{|c|}{ Plasma } & \multicolumn{2}{c|}{ Separated Plasma from whole blood } \\
\hline $\begin{array}{l}\text { Flow resistance } \\
\left(\mathrm{m}^{-3}\right)\end{array}$ & $8.80 \mathrm{E}+17$ & $\begin{array}{l}\text { Flow resistance } \\
\left(\mathrm{m}^{-3}\right)\end{array}$ & $8.80 \mathrm{E}+17$ \\
\hline $\begin{array}{l}\text { Average Velocity } \\
\text { mean } \pm \mathrm{SD}(\mu \mathrm{m} / \mathrm{s})\end{array}$ & $375 \pm 73.54$ & $\begin{array}{l}\text { Average Velocity } \\
\text { mean } \pm \mathrm{SD}(\mu \mathrm{m} / \mathrm{s})\end{array}$ & $385 \pm 24.09$ \\
\hline $\begin{array}{l}\text { Depth of channel } \\
(\mu \mathrm{m})\end{array}$ & 25 & $\begin{array}{l}\text { Depth of channel } \\
(\mu \mathrm{m})\end{array}$ & 25 \\
\hline $\begin{array}{l}\text { Width of channel } \\
(\mu \mathrm{m})\end{array}$ & 200 & $\begin{array}{l}\text { Width of channel } \\
(\mu \mathrm{m})\end{array}$ & 200 \\
\hline Flow rate $(\mathrm{nL} / \mathrm{s})$ & 1.87 & Flow rate $(\mathrm{nL} / \mathrm{s})$ & 1.92 \\
\hline
\end{tabular}




\section{Chapter 6 Conclusions and Future Work}

In order to bridge the healthcare gap between developing and developed countries, there is a growing need for integrated, low cost solutions that are easy to handle, and that are able to provide high quality results. Microfuidics is a powerful alternative for addressing this objective, however, most of current technologies are not fully integrated in-chip, and therefore require external equipment which is expensive and not portable.

The LFD developed in this work is able to overcome this problematic, by integrating all functions in-chip, allowing for the performance of entire laboratory protocols for immunoassays without the need of any external equipment. The LFD combines the ability to separate plasma from a small sample of whole blood, initiate and control the fluid flow taking advantage of capillary forces, and the potential to implement to implement a wide range of immunoassays. The patient would need only to provide a sample droplet of whole blood, and would be able to see the results within the course of several minutes. This platform is sensitive, versatile, and easy to use, which makes it a great alternative for point-of-care testing.

In this work PDMS was functionalized using Pluronic F127, in order to render it hydrophilic. Because time is a critical factor in performing sandwich immunoassays, flow rate was controlled by several factors, including the structures size and position in thee capillary pumps, the concentration of pluronic, and by tuning the resistance of the flow resistor. It was observed from the experiments that the resistance of the flow resistor had the greatest effect on the flow rate of the fluids. Plasma flow rates ranging between 0.43 and $18.60 \mathrm{~nL} / \mathrm{s}$ were achieved in the flow resistor, and between 0.99 and $61.30 \mathrm{~nL} / \mathrm{s}$ in the whole device. Even though water treatment presents an alternative for stabilizing the pluronic and preventing non-specific absorption, it is key to find ways to control evaporation.

Efforts are currently been made to test the device using different detection techniques such as colorimetric and fluorescence, for the detection of Immunoglobulin G (IgG), and traumatic brain injury biomarkers, but the LFD can be used for a diversity of targets.

The current platform is designed for the detection of a single biomarker, but it will be further modified to obtain multiplexing capabilities, which would allow generating more meaningful and conclusive information for clinical diagnosis (Figure 6-1).

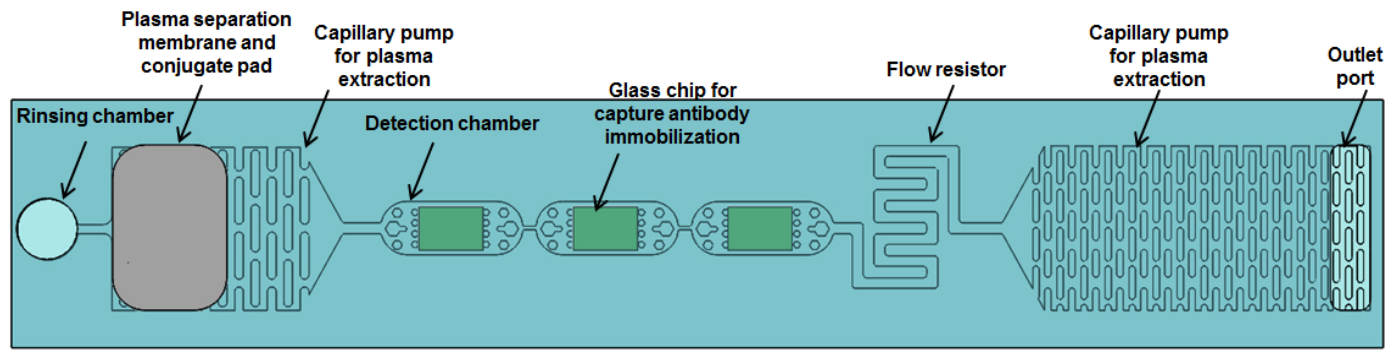

Figure 6-1 Schematic of potential design for a multiplexing platform. 


\section{Bibliography}

[1] D. H. Peters, A. Garg, G. Bloom, D. G. Walker, W. R. Brieger, and M. H. Rahman, "Poverty and access to health care in developing countries," Ann. N. Y. Acad. Sci., vol. 1136, pp. 161$171,2008$.

[2] C. Gouvea, "Biosensors for health applications," in Biosensors for Health, Environment and Biosecurity, P. A. Serra, Ed. InTech, 2011.

[3] L. Gervais, N. de Rooij, and E. Delamarche, "Microfluidic Chips for Point-of-Care Immunodiagnostics," Adv. Mater., vol. 23, no. 24, pp. H151-H176, Jun. 2011.

[4] D. Juncker, "CAPILLARY MICROFLUIDIC SYSTEMS FOR BIO/CHEMISTRY," Universit_e de Neuchatel, Zurich, 2002.

[5] Fluigent, "What is Microfluidics ?" [Online]. Available: http://www.fluigent.com/what-ismicrofluidics/. [Accessed: 29-Jan-2016].

[6] T. Vestad, D. W. M. Marr, and J. Oakey, "Flow control for capillary-pumped microfluidic systems," J. Micromechanics Microengineering, vol. 14, no. 11, pp. 1503-1506, Nov. 2004.

[7] K. Ohno, K. Tachikawa, and A. Manz, "Microfluidics: applications for analytical purposes in chemistry and biochemistry," Electrophoresis, vol. 29, no. 22, pp. 4443-4453, Nov. 2008.

[8] N. S. Lynn and D. S. Dandy, "Passive microfluidic pumping using coupled capillary/evaporation effects," Lab. Chip, vol. 9, no. 23, p. 3422, 2009.

[9] D. Juncker, H. Schmid, U. Drechsler, H. Wolf, M. Wolf, B. Michel, N. de Rooij, and E. Delamarche, "Autonomous Microfluidic Capillary System," Anal. Chem., vol. 74, no. 24, pp. 6139-6144, Dec. 2002.

[10] H. Zhu, M. Bilgin, R. Bangham, D. Hall, A. Casamayor, P. Bertone, N. Lan, R. Jansen, S. Bidlingmaier, T. Houfek, T. Mitchell, P. Miller, R. A. Dean, M. Gerstein, and M. Snyder, "Global analysis of protein activities using proteome chips," Science, vol. 293, no. 5537, pp. 2101-2105, Sep. 2001.

[11] M. A. Burns, "An Integrated Nanoliter DNA Analysis Device," Science, vol. 282, no. 5388, pp. 484-487, Oct. 1998.

[12] [Mu] TAS 2001 Symposium, A. van den Berg, and J. M. Ramsey, Micro total analysis systems 2001 proceedings of the [mu] TAS 2001 Symposium, held in Monterey, CA, USA, 2125 October, 2001. Dordrecht: Springer, 2001.

[13] M. Ahmadi, J. Yeow, and M. Shahini, "System Integration in Microfluidics," in Microfluidics and Nanofluidics Handbook, CRC Press, 2011, pp. 269-286.

[14] T. Matsunaga, M. Hosokawa, A. Arakaki, T. Taguchi, T. Mori, T. Tanaka, and H. Takeyama, "High-efficiency single-cell entrapment and fluorescence in situ hybridization analysis using a poly(dimethylsiloxane) microfluidic device integrated with a black poly(ethylene terephthalate) micromesh," Anal. Chem., vol. 80, no. 13, pp. 5139-5145, Jul. 2008.

[15] J. Zhou, D. A. Khodakov, A. V. Ellis, and N. H. Voelcker, "Surface modification for PDMS-based microfluidic devices," ELECTROPHORESIS, vol. 33, no. 1, pp. 89-104, Jan. 2012.

[16] G. M. Whitesides, "The origins and the future of microfluidics," Nature, vol. 442, no. 7101, pp. 368-373, Jul. 2006.

[17] V. Gubala, L. F. Harris, A. J. Ricco, M. X. Tan, and D. E. Williams, "Point of Care Diagnostics: Status and Future," Anal. Chem., vol. 84, no. 2, pp. 487-515, Jan. 2012. 
[18] L. J. Thomas and S. P. Bessman, "Prototype for an implantable micropump powdered by piezoelectric disk benders," Trans. - Am. Soc. Artif. Intern. Organs, vol. 21, pp. 516-522, 1975.

[19] W. J. Spencer, W. T. Corbett, L. R. Dominguez, and B. D. Shafer, “An electronically controlled piezoelectric insulin pump and valves," IEEE Trans. Sonics Ultrason., vol. 25, no. 3, pp. 153-156, May 1978.

[20] P. Woias, "Micropumps - past, progress and future prospects," Sens. Actuators B Chem., vol. 105 , no. 1 , pp. 28-38, Feb. 2005.

[21] A. Manz, N. Graber, and H. M. Widmer, "Miniaturized total chemical analysis systems: A novel concept for chemical sensing," Sens. Actuators B Chem., vol. 1, no. 1-6, pp. 244248, Jan. 1990.

[22] D. J. Harrison, A. Manz, Z. Fan, H. Luedi, and H. M. Widmer, "Capillary electrophoresis and sample injection systems integrated on a planar glass chip," Anal. Chem., vol. 64, no. 17, pp. 1926-1932, Sep. 1992.

[23] D. J. Harrison, K. Fluri, K. Seiler, Z. Fan, C. S. Effenhauser, and A. Manz, "Micromachining a Miniaturized Capillary Electrophoresis-Based Chemical Analysis System on a Chip," Science, vol. 261, no. 5123, pp. 895-897, Aug. 1993.

[24] D. Issadore and R. M. Westervelt, Eds., Point-of-Care Diagnostics on a Chip. Berlin, Heidelberg: Springer Berlin Heidelberg, 2013.

[25] L. Gervais, "Capillary Microfluidic Chips for Point-of-Care Testing: from Research Tools to Decentralized Medical Diagnostics," ÉCOLE POLYTECHNIQUE FÉDÉRALE DE LAUSANNE, Suisse, 2011.

[26] S. K. Sia and L. J. Kricka, "Microfluidics and point-of-care testing," Lab. Chip, vol. 8, no. 12, p. 1982, 2008.

[27] U. Demirci, Ed., Microfluidic technologies for human health. New Jersey : London: World Scientific Pub. ; Imperial College Press, 2013.

[28] D. A. Hall, J. Ptacek, and M. Snyder, "Protein microarray technology," Mech. Ageing Dev., vol. 128, no. 1, pp. 161-167, Jan. 2007.

[29] M.-I. Mohammed and M. P. Y. Desmulliez, "Lab-on-a-chip based immunosensor principles and technologies for the detection of cardiac biomarkers: a review," Lab Chip, vol. 11, no. 4, pp. 569-595, 2011.

[30] L. Wu, C. Ma, L. Ge, Q. Kong, M. Yan, S. Ge, and J. Yu, "Paper-based electrochemiluminescence origami cyto-device for multiple cancer cells detection using porous AuPd alloy as catalytically promoted nanolabels," Biosens. Bioelectron., vol. 63, pp. 450-457, Jan. 2015.

[31] Y. Wu, P. Xue, K. M. Hui, and Y. Kang, "A paper-based microfluidic electrochemical immunodevice integrated with amplification-by-polymerization for the ultrasensitive multiplexed detection of cancer biomarkers," Biosens. Bioelectron., vol. 52, pp. 180-187, Feb. 2014.

[32] S. Wang, L. Ge, X. Song, J. Yu, S. Ge, J. Huang, and F. Zeng, "Paper-based chemiluminescence ELISA: lab-on-paper based on chitosan modified paper device and waxscreen-printing," Biosens. Bioelectron., vol. 31, no. 1, pp. 212-218, Jan. 2012.

[33] P. Wang, L. Ge, M. Yan, X. Song, S. Ge, and J. Yu, "Paper-based three-dimensional electrochemical immunodevice based on multi-walled carbon nanotubes functionalized paper for sensitive point-of-care testing," Biosens. Bioelectron., vol. 32, no. 1, pp. 238-243, Feb. 2012. 
[34] W. Li, M. Li, S. Ge, M. Yan, J. Huang, and J. Yu, "Battery-triggered ultrasensitive electrochemiluminescence detection on microfluidic paper-based immunodevice based on dual-signal amplification strategy," Anal. Chim. Acta, vol. 767, pp. 66-74, Mar. 2013.

[35] S. Wang, L. Ge, M. Yan, J. Yu, X. Song, S. Ge, and J. Huang, "3D microfluidic origami electrochemiluminescence immunodevice for sensitive point-of-care testing of carcinoma antigen 125," Sens. Actuators B Chem., vol. 176, pp. 1-8, Jan. 2013.

[36] N. P. Sardesai, K. Kadimisetty, R. Faria, and J. F. Rusling, "A microfluidic electrochemiluminescent device for detecting cancer biomarker proteins," Anal. Bioanal. Chem., vol. 405, no. 11, pp. 3831-3838, Apr. 2013.

[37] X. Zhou, T. I. Wong, H. Y. Song, L. Wu, Y. Wang, P. Bai, D.-H. Kim, S. H. Ng, M. S. Tse, and W. Knoll, "Development of Localized Surface Plasmon Resonance-Based Point-ofCare System," Plasmonics, vol. 9, no. 4, pp. 835-844, Aug. 2014.

[38] S. T. Sanjay, G. Fu, M. Dou, F. Xu, R. Liu, H. Qi, and X. Li, "Biomarker detection for disease diagnosis using cost-effective microfluidic platforms," The Analyst, vol. 140, no. 21, pp. 7062-7081, 2015.

[39] S. T. Sanjay, G. Fu, M. Dou, F. Xu, R. Liu, H. Qi, and X. Li, "Biomarker detection for disease diagnosis using cost-effective microfluidic platforms," The Analyst, vol. 140, no. 21, pp. 7062-7081, 2015.

[40] K.-W. Chang, J. Li, C.-H. Yang, S.-C. Shiesh, and G.-B. Lee, “An integrated microfluidic system for measurement of glycated hemoglobin levels by using an aptamerantibody assay on magnetic beads," Biosens. Bioelectron., vol. 68, pp. 397-403, Jun. 2015.

[41] K.-W. Chang, J.-L. Li, C.-H. Yang, S.-C. Shiesh, and G.-B. Lee, "Measurement of glycated hemoglobin using an aptamer/antibody assay on an integrated microfluidic system," 2014, pp. 647-650.

[42] C.-C. Wu, H.-I. Lin, K.-W. Chang, J. D. Mai, S.-C. Shiesh, and G.-B. Lee, "Measurement of glycated hemoglobin levels using an integrated microfluidic system," Microfluid. Nanofluidics, vol. 18, no. 4, pp. 613-621, Apr. 2015.

[43] J.-W. Choi, K. W. Oh, J. H. Thomas, W. R. Heineman, H. B. Halsall, J. H. Nevin, A. J. Helmicki, H. T. Henderson, and C. H. Ahn, "An integrated microfluidic biochemical detection system for protein analysis with magnetic bead-based sampling capabilities," Lab. Chip, vol. 2, no. 1, p. 27, 2002.

[44] S. Wang, L. Ge, X. Song, J. Yu, S. Ge, J. Huang, and F. Zeng, "Paper-based chemiluminescence ELISA: lab-on-paper based on chitosan modified paper device and waxscreen-printing," Biosens. Bioelectron., vol. 31, no. 1, pp. 212-218, Jan. 2012.

[45] E. Anderson, M. Conway, H. Alloush, K. O’Malley, M. A. Smith, A. Martin, M. Ruddock, C. Reid, J. Lamont, S. P. Fitzgerald, J. G. Smith, P. Mehta, and V. Salisbury, "Investigation and verification of a bioluminescent biosensor for the quantitation of ara-CTP generation: a biomarker for cytosine arabinoside sensitivity in acute myeloid leukaemia," Biosens. Bioelectron., vol. 52, pp. 345-353, Feb. 2014.

[46] N. V. Zaytseva, R. A. Montagna, and A. J. Baeumner, "Microfluidic Biosensor for the Serotype-Specific Detection of Dengue Virus RNA," Anal. Chem., vol. 77, no. 23, pp. 75207527, Dec. 2005.

[47] A. J. Baeumner, N. A. Schlesinger, N. S. Slutzki, J. Romano, E. M. Lee, and R. A. Montagna, "Biosensor for Dengue Virus Detection: Sensitive, Rapid, and Serotype Specific," Anal. Chem., vol. 74, no. 6, pp. 1442-1448, Mar. 2002. 
[48] Y.-F. Lee, K.-Y. Lien, H.-Y. Lei, and G.-B. Lee, “An integrated microfluidic system for rapid diagnosis of dengue virus infection," Biosens. Bioelectron., vol. 25, no. 4, pp. 745-752, Dec. 2009.

[49] M. Dou, D. C. Dominguez, X. Li, J. Sanchez, and G. Scott, “A versatile PDMS/paper hybrid microfluidic platform for sensitive infectious disease diagnosis," Anal. Chem., vol. 86, no. 15, pp. 7978-7986, Aug. 2014.

[50] J. Pipper, M. Inoue, L. F.-P. Ng, P. Neuzil, Y. Zhang, and L. Novak, "Catching bird flu in a droplet," Nat. Med., vol. 13, no. 10, pp. 1259-1263, Nov. 2007.

[51] M. Hu, J. Yan, Y. He, H. Lu, L. Weng, S. Song, C. Fan, and L. Wang, "Ultrasensitive, Multiplexed Detection of Cancer Biomarkers Directly in Serum by Using a Quantum DotBased Microfluidic Protein Chip," ACS Nano, vol. 4, no. 1, pp. 488-494, Jan. 2010.

[52] Y.-F. Lee, K.-Y. Lien, H.-Y. Lei, and G.-B. Lee, "An integrated microfluidic system for rapid diagnosis of dengue virus infection," Biosens. Bioelectron., vol. 25, no. 4, pp. 745-752, Dec. 2009.

[53] D. Maji, M. A. Suster, E. Stavrou, U. A. Gurkan, and P. Mohseni, "Monitoring time course of human whole blood coagulation using a microfluidic dielectric sensor with a 3D capacitive structure," Conf. Proc. Annu. Int. Conf. IEEE Eng. Med. Biol. Soc. IEEE Eng. Med. Biol. Soc. Annu. Conf., vol. 2015, pp. 5904-5907, Aug. 2015.

[54] K. Länge, B. E. Rapp, and M. Rapp, "Surface acoustic wave biosensors: a review," Anal. Bioanal. Chem., vol. 391, no. 5, pp. 1509-1519, Jul. 2008.

[55] X. Zhang, Y. Zou, C. An, K. Ying, X. Chen, and P. Wang, "A miniaturized immunosensor platform for automatic detection of carcinoembryonic antigen in EBC," Sens. Actuators B Chem., vol. 205, pp. 94-101, Dec. 2014.

[56] T. M. A. Gronewold, "Surface acoustic wave sensors in the bioanalytical field: Recent trends and challenges," Anal. Chim. Acta, vol. 603, no. 2, pp. 119-128, Nov. 2007.

[57] D. Vilela, M. C. González, and A. Escarpa, "Sensing colorimetric approaches based on gold and silver nanoparticles aggregation: chemical creativity behind the assay. A review," Anal. Chim. Acta, vol. 751, pp. 24-43, Nov. 2012.

[58] W. Qu, Y. Liu, D. Liu, Z. Wang, and X. Jiang, "Copper-Mediated Amplification Allows Readout of Immunoassays by the Naked Eye," Angew. Chem., vol. 123, no. 15, pp. 35043507, Apr. 2011.

[59] "How Pregnancy Tests Work." [Online]. Available: http://chemistry.about.com/od/chemistryfaqs/f/pregnancytest.htm. [Accessed: 14-Jul-2014].

[60] "Pregnancy Test Strip Instructions." [Online]. Available: http://www.early-pregnancytests.com/inpregtesstr.html. [Accessed: 14-Jul-2014].

[61] L. Yu, C. M. Li, Y. Liu, J. Gao, W. Wang, and Y. Gan, "Flow-through functionalized PDMS microfluidic channels with dextran derivative for ELISAs," Lab. Chip, vol. 9, no. 9, p. $1243,2009$.

[62] C. D. Chin, T. Laksanasopin, Y. K. Cheung, D. Steinmiller, V. Linder, H. Parsa, J. Wang, H. Moore, R. Rouse, G. Umviligihozo, E. Karita, L. Mwambarangwe, S. L. Braunstein, J. van de Wijgert, R. Sahabo, J. E. Justman, W. El-Sadr, and S. K. Sia, "Microfluidics-based diagnostics of infectious diseases in the developing world," Nat. Med., vol. 17, no. 8, pp. 1015-1019, Jul. 2011.

[63] W. Du, L. Li, K. P. Nichols, and R. F. Ismagilov, "SlipChip,” Lab. Chip, vol. 9, no. 16, p. 2286, 2009. 
[64] “3D Printed Diagnostic Device Rapidly Detects Anemia.” [Online]. Available: http://www.mdtmag.com/news/2016/02/3d-printed-diagnostic-device-rapidly-detects-anemia. [Accessed: 03-Mar-2016].

[65] E. Petryayeva and U. J. Krull, "Localized surface plasmon resonance: Nanostructures, bioassays and biosensing-A review," Anal. Chim. Acta, vol. 706, no. 1, pp. 8-24, Nov. 2011.

[66] T. Lai, Q. Hou, H. Yang, X. Luo, and M. Xi, "Clinical application of a novel sliver nanoparticles biosensor based on localized surface plasmon resonance for detecting the microalbuminuria," Acta Biochim. Biophys. Sin., vol. 42, no. 11, pp. 787-792, Nov. 2010.

[67] P. Liu, X. Li, S. A. Greenspoon, J. R. Scherer, and R. A. Mathies, "Integrated DNA purification, PCR, sample cleanup, and capillary electrophoresis microchip for forensic human identification," Lab. Chip, vol. 11, no. 6, pp. 1041-1048, Mar. 2011.

[68] B. S. Lee, Y. U. Lee, H.-S. Kim, T.-H. Kim, J. Park, J.-G. Lee, J. Kim, H. Kim, W. G. Lee, and Y.-K. Cho, "Fully integrated lab-on-a-disc for simultaneous analysis of biochemistry and immunoassay from whole blood," Lab Chip, vol. 11, no. 1, pp. 70-78, 2011.

[69] J. Nguyen, Y. Wei, Y. Zheng, C. Wang, and Y. Sun, "On-chip sample preparation for complete blood count from raw blood," Lab. Chip, vol. 15, no. 6, pp. 1533-1544, Mar. 2015.

[70] X. Mao and T. J. Huang, "Microfluidic diagnostics for the developing world," Lab. Chip, vol. 12, no. 8, p. 1412, 2012.

[71] E A BOUCHER, "Capillary phenomena: Properties of systems with fluid/fluid interfaces," University of Sussex, UK, 1980.

[72] Aleksandr Mikhaïlovich Prokhorov, "Capillary Phenomena," Great Soviet Encyclopedia. 1979.

[73] Nelli, Giovan Battista, "Saggio di storia letteraria fiorentina del secolo XVII," 1759.

[74] R. Boyle, New experiments physico-mechanicall, touching the spring of the air, and its effects. 1660.

[75] J. C. Sturm, Collegium Experimentale sive Curiosum, vol. 1. University of Lausanne, 1676.

[76] G. Sinclair, Ars nova et magna gravitatis et levitatis. .

[77] Montanari, Geminiano, "Pensieri fisico-matematici sopra alcune esperienze fatte in Bologna nell'Accademia Filosofica... intorno diversi effetti de' liquidi in cannuccie di vetro et altri vasi," 1667.

[78] R. Hooke, Octavo Corporation, and Warnock Library, Micrographia. Palo Alto, Ca.: Octavo, 1998.

[79] R. Hooke, An attempt for the explication of the phoenomena observable in an experiment published by the Honourable Robert Boyle, Esq., in the XXXV experiment of his epistolical discourse touching the aire in confirmation of a former conjecture made by R.H. Ann Arbor, $\mathrm{MI}$; Oxford (UK).

[80] J. Bernoulli, Jacobi Bernoulli Dissertatio de gravitate oetheris. Apud H. Wetstenium, 1683.

[81] Honoré Fabri, Dialogi physici, in quibus de motu Terrae disputatur, marini aestus nova causa proponitur. Sumptibus Christophori Fourmy, in vico Mercatorio, sub signo Occasionis, 1665.

[82] I. Vossius, De Nili et aliorum fluminum origine. Universiteit Gent: Ex typographia Adriani Vlacq, 1666. 
[83] L. Carré, Experiences sur les tuyaux Capillaires. 1705.

[84] G. A. Borelli, De Motionibus Naturalibus, A Gravitate Pendentibus. Lugduni Batavorum//Leiden: Apud Petrum Vander Aa, 1686.

[85] F. Hauksbee, "Several Experiments Touching the Seeming Spontaneous Ascent of Water. By Mr. Fr. Hauksbee, F. R. S.," Philos. Trans. R. Soc. Lond., vol. 26, no. 313-324, pp. 258266, Jan. 1708.

[86] Hauksbee, Francis, "Physico-mechanical experiments on various subjects containing an account of several surprizing phenomena touching light and electricity, producible on the attrition of bodies : with many other remarkable appearances, not before observ'd : together with the explanations of all the machines, (the figures of which are curiously engrav'd on copper) and other apparatus us'd in making the experiments," 1709.

[87] F. Hauksbee, "An Account of an Experiment Touching the Direction of a Drop of Oil of Oranges, between Two Glass Planes, towards Any Side of Them That is Nearest Press'd Together. By Mr. Fr. Hauksbee, F. R. S.," Philos. Trans. R. Soc. Lond., vol. 27, no. 325-336, pp. 395-396, Jan. 1710.

[88] J. Weitbrecht, Tentamen theoriae qua ascensus aquae in tubis capillaribus explicatur, vol. 8. 1736.

[89] J. Weitbrecht, Explicatio difficilium experimentorum circa ascensum aquae in tubis capillaribus, vol. 9. 1737.

[90] T. Young, "An Essay on the Cohesion of Fluids," Philos. Trans. R. Soc. Lond., vol. 95, no. 0, pp. 65-87, Jan. 1805.

[91] Pierre Simon marquis de Laplace, Traité de Mécanique Céleste, vol. 4. Paris, 1805.

[92] C. F. Gauss, Principia generalia Theoriae Figurae Fluidorum in statu Aequilibrii. Germany, 1830.

[93] F. Neumann and A. Wangerin, Vorlesungen über die Theorie der Capillarität. Germany, 1894.

[94] W. Thomson, "On the equilibrium of vapour at a curved surface of liquid," Philosophical Magazine, pp. 448-452, 1871.

[95] J. N. Israelachvili, Intermolecular and surface forces, 3rd ed. Burlington, MA: Academic Press, 2011.

[96] P. G. de Gennes, "Wetting: statics and dynamics," Rev. Mod. Phys., vol. 57, no. 3, pp. 827-863, Jul. 1985.

[97] M. Nosonovsky and R. Ramachandran, "Geometric Interpretation of Surface Tension Equilibrium in Superhydrophobic Systems," Entropy, vol. 17, no. 7, pp. 4684-4700, Jul. 2015.

[98] M. Zimmermann, H. Schmid, P. Hunziker, and E. Delamarche, "Capillary pumps for autonomous capillary systems," Lab. Chip, vol. 7, no. 1, p. 119, 2007.

[99] G. A. Urban, Ed., BioMEMS. Dordrecht: Springer, 2006.

[100] J. P. Brody, P. Yager, R. E. Goldstein, and R. H. Austin, "Biotechnology at low Reynolds numbers," Biophys. J., vol. 71, no. 6, pp. 3430-3441, Dec. 1996.

[101] T. M. Squires and S. R. Quake, "Microfluidics: Fluid physics at the nanoliter scale," Rev. Mod. Phys., vol. 77, no. 3, pp. 977-1026, Oct. 2005.

[102] A. Biral, "Microfluidic networking: modelling and analysis," UNIVERSITA DEGLI STUDI DI PADOVA, 2012.

[103] "Viscosity - The Physics Hypertextbook." [Online]. Available: http://physics.info/viscosity/. [Accessed: 14-Feb-2016]. 
[104] "Viscosity - Wikipedia, the free encyclopedia." [Online]. Available:

https://en.wikipedia.org/wiki/Viscosity. [Accessed: 14-Feb-2016].

[105] Meridian Valley Lab, "Whole Blood Viscosity vs. Serum and Plasma Viscosity."

[Online]. Available: http://meridianvalleylab.com/whole-blood-viscosity-vs-serum-and-

plasma-viscosity. [Accessed: 03-Feb-2015].

[106] G. Késmárky, P. Kenyeres, M. Rábai, and K. Tóth, "Plasma viscosity: a forgotten

variable," Clin. Hemorheol. Microcirc., vol. 39, no. 1-4, pp. 243-246, 2008.

[107] "biofilm_doseresponse_pg1 - 168_BioFlux_Viscosity_TechNote-1038-01.pdf.".

[108] "POISEÜILLE'S LAW AND THE VISCOSITY OF FLUIDS." [Online]. Available:

http://galileo.phys.virginia.edu/classes/241L/poise/poise.htm. [Accessed: 15-Feb-2016].

[109] F. Rodriguez, "The analogy between fluid flow and electric circuitry," Ithaca, New York.

[110] Hutchinson (Firm), The Hutchinson unabridged encyclopedia with atlas and weather guide. Oxford, England: Helicon, 2010.

[111] D. J. Beebe, G. A. Mensing, and G. M. Walker, "Physics and Applications of

Microfluidics in Biology," Annu. Rev. Biomed. Eng., vol. 4, no. 1, pp. 261-286, Aug. 2002.

[112] H. van Heeren and P. Hewkin, "Design for Microfluidic Device Manufacture Guidelines." 05-Apr-2014.

[113] G. M. Whitesides, "The origins and the future of microfluidics," Nature, vol. 442, no. 7101, pp. 368-373, Jul. 2006.

[114] J. P. Brody, P. Yager, R. E. Goldstein, and R. H. Austin, "Biotechnology at low Reynolds numbers," Biophys. J., vol. 71, no. 6, pp. 3430-3441, Dec. 1996.

[115] L. A. Godwin, K. S. Deal, L. D. Hoepfner, L. A. Jackson, and C. J. Easley, "Measurement of microchannel fluidic resistance with a standard voltage meter," Anal. Chim. Acta, vol. 758, pp. 101-107, Jan. 2013.

[116] P. Abgrall and A.-M. Gué, "Lab-on-chip technologies: making a microfluidic network and coupling it into a complete microsystem - a review," J. Micromechanics

Microengineering, vol. 17, no. 5, pp. R15-R49, May 2007.

[117] K. Ren, J. Zhou, and H. Wu, "Materials for Microfluidic Chip Fabrication," Acc. Chem. Res., vol. 46, no. 11, pp. 2396-2406, Nov. 2013.

[118] "Microfluidics? A general overview." [Online]. Available:

http://www.elveflow.com/microfluidic-tutorials/microfluidic-reviews-andtutorials/microfluidics/. [Accessed: 26-Mar-2016].

[119] K. F. Lei, "Chapter 1. Materials and Fabrication Techniques for Nano- and Microfluidic Devices," in RSC Detection Science, F. H. Labeed and H. O. Fatoyinbo, Eds. Cambridge: Royal Society of Chemistry, 2014, pp. 1-28.

[120] A. W. Martinez, S. T. Phillips, G. M. Whitesides, and E. Carrilho, "Diagnostics for the Developing World: Microfluidic Paper-Based Analytical Devices," Anal. Chem., vol. 82, no. 1, pp. 3-10, Jan. 2010.

[121] R. G. Blazej, P. Kumaresan, and R. A. Mathies, "Microfabricated bioprocessor for integrated nanoliter-scale Sanger DNA sequencing," Proc. Natl. Acad. Sci., vol. 103, no. 19, pp. 7240-7245, May 2006.

[122] T. G. Henares, F. Mizutani, and H. Hisamoto, "Current development in microfluidic immunosensing chip," Anal. Chim. Acta, vol. 611, no. 1, pp. 17-30, Mar. 2008.

[123] K. Kalkandjiev, L. Riegger, D. Kosse, M. Welsche, L. Gutzweiler, R. Zengerle, and P. Koltay, "Microfluidics in silicon/polymer technology as a cost-efficient alternative to silicon/glass," J. Micromechanics Microengineering, vol. 21, no. 2, p. 25008, Feb. 2011. 
[124] M. Grundner and H. Jacob, "Investigations on hydrophilic and hydrophobic silicon (100) wafer surfaces by X-ray photoelectron and high-resolution electron energy lossspectroscopy," Appl. Phys. Solids Surf., vol. 39, no. 2, pp. 73-82, Feb. 1986.

[125] W. Kern and D.A. Puotinen, "Cleaning Solutions Based on Hydrogen Peroxide for Use in Silicon Semiconductor Technology,” vol. 31, pp. 187-206, 1970.

[126] B. R. Benson, H. A. Stone, and R. K. Prud'homme, “An 'off-the-shelf' capillary microfluidic device that enables tuning of the droplet breakup regime at constant flow rates," Lab. Chip, vol. 13, no. 23, p. 4507, 2013.

[127] Z. Chang, C. A. Serra, M. Bouquey, L. Prat, and G. Hadziioannou, "Co-axial capillaries microfluidic device for synthesizing size- and morphology-controlled polymer core-polymer shell particles," Lab. Chip, vol. 9, no. 20, pp. 3007-3011, Oct. 2009.

[128] W. Wang, R. Xie, X.-J. Ju, T. Luo, L. Liu, D. A. Weitz, and L.-Y. Chu, "Controllable microfluidic production of multicomponent multiple emulsions," Lab. Chip, vol. 11, no. 9, pp. 1587-1592, May 2011.

[129] X. Li, D. R. Ballerini, and W. Shen, “A perspective on paper-based microfluidics: Current status and future trends," Biomicrofluidics, vol. 6, no. 1, p. 11301, 2012.

[130] R. H. Müller and D. L. Clegg, "Automatic Paper Chromatography," Anal. Chem., vol. 21, no. 9, pp. 1123-1125, Sep. 1949.

[131] J. C. McDonald, D. C. Duffy, J. R. Anderson, D. T. Chiu, H. Wu, O. J. Schueller, and G. M. Whitesides, "Fabrication of microfluidic systems in poly(dimethylsiloxane)," Electrophoresis, vol. 21, no. 1, pp. 27-40, Jan. 2000.

[132] Y. Zhu and K. Petkovic-Duran, "\&lt;title\&gt;Capillary flow in polymer microfluidic chips\&lt;/title\&gt;", 2006, p. 64160K-64160K-6.

[133] E. K. Sackmann, A. L. Fulton, and D. J. Beebe, "The present and future role of microfluidics in biomedical research," Nature, vol. 507, no. 7491, pp. 181-189, Mar. 2014.

[134] K. J. Regehr, M. Domenech, J. T. Koepsel, K. C. Carver, S. J. Ellison-Zelski, W. L. Murphy, L. A. Schuler, E. T. Alarid, and D. J. Beebe, "Biological implications of polydimethylsiloxane-based microfluidic cell culture," Lab. Chip, vol. 9, no. 15, pp. 21322139, Aug. 2009.

[135] M. W. Toepke and D. J. Beebe, "PDMS absorption of small molecules and consequences in microfluidic applications," Lab. Chip, vol. 6, no. 12, pp. 1484-1486, Dec. 2006.

[136] E. Berthier, J. Warrick, H. Yu, and D. J. Beebe, "Managing evaporation for more robust microscale assays. Part 2. Characterization of convection and diffusion for cell biology," Lab. Chip, vol. 8, no. 6, pp. 860-864, Jun. 2008.

[137] J. Zhou, A. V. Ellis, and N. H. Voelcker, "Recent developments in PDMS surface modification for microfluidic devices," ELECTROPHORESIS, vol. 31, no. 1, pp. 2-16, Jan. 2010.

[138] W. Qiu, X. Sun, C. Wu, K. Hjort, and Z. Wu, “A Contact Angle Study of the Interaction between Embedded Amphiphilic Molecules and the PDMS Matrix in an Aqueous Environment," Micromachines, vol. 5, no. 3, pp. 515-527, Aug. 2014.

[139] Z. Wu and K. Hjort, "Surface modification of PDMS by gradient-induced migration of embedded Pluronic," Lab. Chip, vol. 9, no. 11, p. 1500, 2009.

[140] P. Alexandridis and T. Alan Hatton, "Poly(ethylene oxide) $\square$ poly(propylene oxide) $\square$ poly(ethylene oxide) block copolymer surfactants in aqueous solutions and at interfaces: thermodynamics, structure, dynamics, and modeling," Colloids Surf. Physicochem. Eng. Asp., vol. 96, no. 1-2, pp. 1-46, Mar. 1995. 
[141] D. C. Duffy, J. C. McDonald, O. J. A. Schueller, and G. M. Whitesides, "Rapid Prototyping of Microfluidic Systems in Poly(dimethylsiloxane)," Anal. Chem., vol. 70, no. 23, pp. 4974-4984, Dec. 1998.

[142] D. J. Laser and J. G. Santiago, “A review of micropumps,” J. Micromechanics Microengineering, vol. 14, no. 6, pp. R35-R64, Jun. 2004.

[143] A. Homsy, "Design, Microfabrication, and Characterization of MHD Pumps and their Applications in NMR Environments," University of Neuchâtel, Switzerland, 2006.

[144] M. Gad-el-Hak, Flow control: passive, active, and reactive flow management, 1. publ., Digitally printed first paperback version. Cambridge: Cambridge Univ. Press, 2006.

[145] E. Berthier and D. J. Beebe, "Flow rate analysis of a surface tension driven passive micropump," Lab. Chip, vol. 7, no. 11, pp. 1475-1478, Nov. 2007.

[146] Y. Zhu and K. Petkovic-Duran, "Capillary flow in microchannels," Microfluid. Nanofluidics, vol. 8, no. 2, pp. 275-282, Feb. 2010.

[147] W. Qiu, X. Sun, C. Wu, K. Hjort, and Z. Wu, “A Contact Angle Study of the Interaction between Embedded Amphiphilic Molecules and the PDMS Matrix in an Aqueous Environment," Micromachines, vol. 5, no. 3, pp. 515-527, Aug. 2014.

[148] A. A. Saha, S. K. Mitra, M. Tweedie, S. Roy, and J. McLaughlin, "Experimental and numerical investigation of capillary flow in SU8 and PDMS microchannels with integrated pillars," Microfluid. Nanofluidics, vol. 7, no. 4, pp. 451-465, Oct. 2009.

[149] M. Zimmermann, H. Schmid, P. Hunziker, and E. Delamarche, "Capillary pumps for autonomous capillary systems," Lab Chip, vol. 7, no. 1, pp. 119-125, 2007.

[150] D. Myers, Surfaces, Interfaces, and Colloids. New York, USA: John Wiley \& Sons, Inc., 1999.

[151] A. Nabatiyan, Z. A. Parpia, R. Elghanian, and D. M. Kelso, "Membrane-based plasma collection device for point-of-care diagnosis of HIV," J. Virol. Methods, vol. 173, no. 1, pp. 37-42, Apr. 2011.

[152] "Vivid ${ }^{\mathrm{TM}}$ Plasma Separation Membrane." [Online]. Available:

http://www.pall.com/main/oem-materials-and-devices/product.page?id=46962. [Accessed:

12-Jun-2015].

[153] M. Gad-el-Hak, The MEMS handbook. Boca Raton, FL: CRC Press, 2002.

[154] A. van den Berg and L. Segerink, Eds., Microfluidics for medical applications. Cambridge, UK: Royal Society of Chemistry, 2015.

[155] A. W. Martinez, S. T. Phillips, M. J. Butte, and G. M. Whitesides, "Patterned Paper as a Platform for Inexpensive, Low-Volume, Portable Bioassays," Angew. Chem. Int. Ed., vol. 46, no. 8, pp. 1318-1320, Feb. 2007.

[156] A. W. Martinez, S. T. Phillips, and G. M. Whitesides, "Three-dimensional microfluidic devices fabricated in layered paper and tape," Proc. Natl. Acad. Sci., vol. 105, no. 50, pp. 19606-19611, Dec. 2008.

[157] N. K. Thom, K. Yeung, M. B. Pillion, and S. T. Phillips, "Fluidic batteries' as low-cost sources of power in paper-based microfluidic devices," Lab. Chip, vol. 12, no. 10, p. 1768, 2012.

[158] N. R. Pollock, J. P. Rolland, S. Kumar, P. D. Beattie, S. Jain, F. Noubary, V. L. Wong, R. A. Pohlmann, U. S. Ryan, and G. M. Whitesides, "A Paper-Based Multiplexed Transaminase Test for Low-Cost, Point-of-Care Liver Function Testing," Sci. Transl. Med., vol. 4, no. 152, pp. 152ra129-152ra129, Sep. 2012. 